\title{
PREDICTING ARTICULAR CARTILAGE CONSTITUENT MATERIAL PROPERTIES FOLLOWING IN VITRO GROWTH USING A PROTEOGLYCAN-COLLAGEN MIXTURE MODEL
}

\author{
A Thesis \\ Presented to the Faculty of the College of Engineering \\ California Polytechnic State University, \\ San Luis Obispo
}

\author{
In Partial Fulfillment \\ of the Requirements for the Degree \\ Master of Science in Mechanical Engineering
}

by

Michael E. Stender

March, 2011 
(C) 2011

Michael E. Stender

ALL RIGHTS RESERVED 


\section{COMMITTEE MEMBERSHIP}

TITLE:

AUTHOR:

DATE SUBMITTED:

March, 2011 MODEL
PREDICTING ARTICULAR CARTILAGE CONSTITUENT MATERIAL PROPERTIES FOLLOWING IN VITRO GROWTH USING A PROTEOGLYCAN-COLLAGEN MIXTURE

Michael E. Stender

COMMITTEE CHAIR: Stephen M. Klisch, Professor

COMMITTEE MEMBER: Scott Hazelwood, Associate Professor

COMMITTEE MEMBER: Andrew Davol, Professor 


\title{
ABSTRACT
}

\author{
PREDICTING ARTICULAR CARTILAGE CONSTITUENT MATERIAL \\ PROPERTIES FOLLOWING IN VITRO GROWTH USING A PROTEOGLYCAN- \\ COLLAGEN MIXTURE MODEL
}

\author{
Michael E. Stender
}

\begin{abstract}
A polyconvex continuum-level proteoglycan Cauchy stress function was developed based on the continuum electromechanical PoissonBoltzmann cell model for proteoglycan interactions. The resulting proteoglycan model was combined with a novel collagen fibril model and a ground substance matrix material to create a polyconvex constitutive finite element model of articular cartilage. The true collagen fibril modulus $\mathrm{E}_{\mathrm{f}}$, and the ground substance matrix shear modulus $\mu$, were varied to obtain the best fit to experimental tension, confined compression, and unconfined compression data for native explants and explants cultured in insulin-like growth factor-1 (IGF-1) and transforming growth factor- $\beta 1$ (TGF- $\beta 1$ ). Results indicate that culture in IGF-1 results in a weakening of the COL fibers compared to native explants, and culture in TGF- $\beta 1$ results in a strengthening of the $\mathrm{COL}$ fibers compared to native explants. These results elucidate the biomechanical changes in collagen fibril modulus, and ground matrix shear modulus following in vitro culture with IGF-1 and TGF- $\beta 1$. Understanding the constitutive effects of growth factor stimulated culture may have applications in AC repair and tissue engineering.
\end{abstract}

Keywords: Articular Cartilage, Finite Element Modeling, Cartilage growth, collagen fiber modulus. 


\section{ACKNOWLEDGMENTS}

I would like to express my sincere gratitude to my committee chair Dr. Stephen Klisch, and my committee member Dr. Scott Hazelwood for their thoughtful and sincere guidance of this project and of my professional development. I am very grateful to have been able to benefit from their experience and I sincerely appreciate the time that they spent in close contact with me during my time in the Cal Poly Cartilage Biomechanics Group. I would also like to express my gratitude to my committee member Dr. Andrew Davol for his willingness to become a part of this project and for sharing his thoughtful and kind advice over the past few years.

During the development of this project I have been very fortunate to have been able to work with members of UCSD's CTE lab. I would like to sincerely thank Dr. Robert Sah, Dr. Greg Williams, and Dr. Albert Chen for their mentorship, assistance, and guidance during my time at the CTE lab, and after.

I am lucky to have been a part of the Cal Poly Cartilage Biomechanics Group. It has been a true pleasure to work with the passionate intelligent students also involved with this group. Thanks to Kevin, Kristin, and David for laughing and working with me in San Diego and to Christian, Britta, and Nathan, without your work and the data you collected none of this would have been possible. I would also like to thank Matt and Kevin for helping review this thesis. Thanks to Kelsey, I don't know what I would've done if there hadn't been anyone around that understood the agony that Abaqus can invoke. I wish you all the best in the future.

Last, but not least I would like to thank Mom, Dad, Nicole, and my other family members that have provided their unfailing support of my education and my dreams. Thanks to Marissa for keeping a smile on my face and being an amazing person. Thanks to Kent, Drew, Matt, and Marisa for all of your support, kindness and your willingness to escape for a weekend of climbing or other adventures.

This project was supported by grants from NSF and NIH. 


\section{TABLE OF CONTENTS}

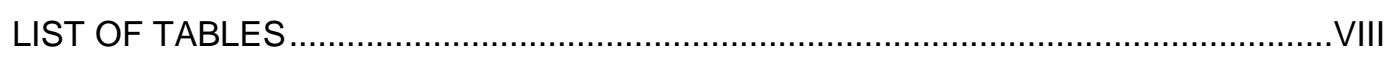

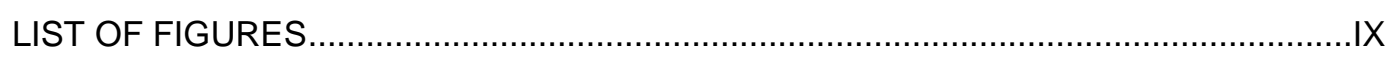

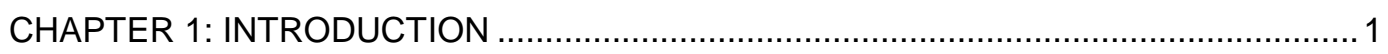

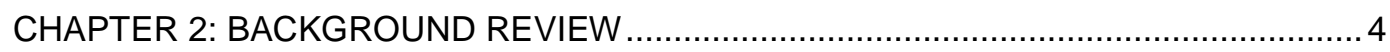

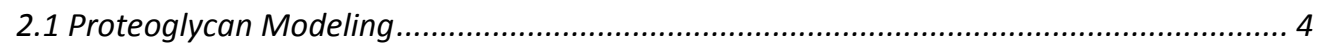

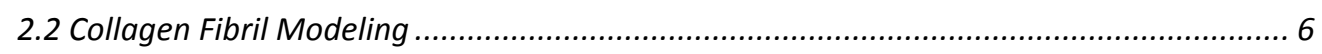

2.3 Ground Substance Matrix Modeling ....................................................................... 8

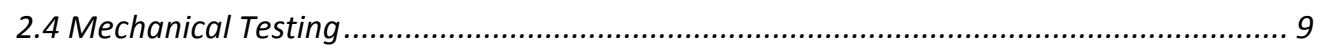

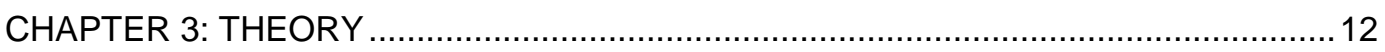



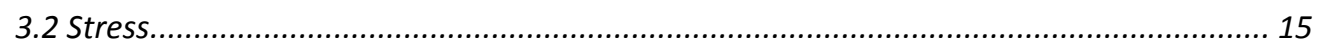

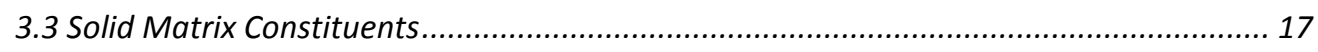

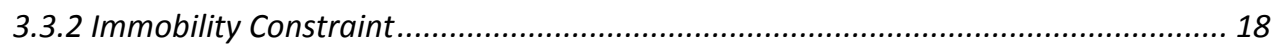



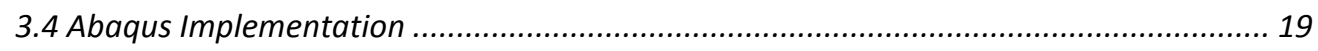

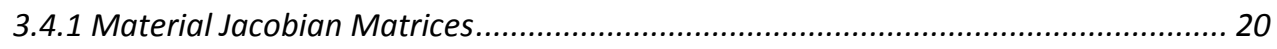

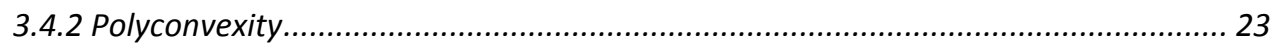

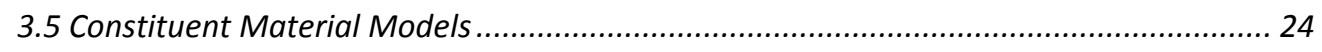



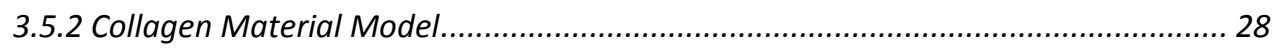

3.5.3 Ground Substance Matrix Material Model........................................................... 34

CHAPTER 4: PROTEOGLYCAN CAUCHY STRESS FUNCTION AND FE

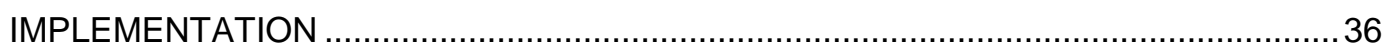

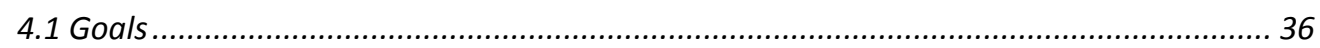

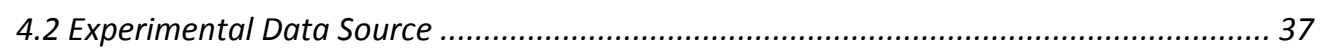

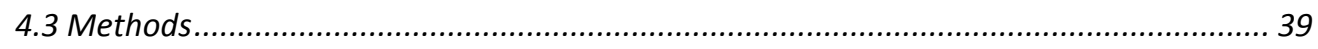

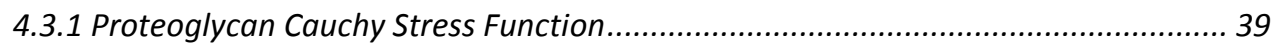

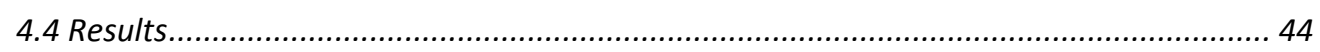

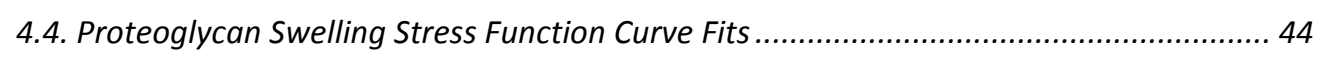

4.4.2 Constituent Stress Predictions in Compression.................................................. 46



4.5.1 PG Swelling Stress Function Curve Fits ............................................................. 49

4.5.2 Constituent Stress Predictions in Confined and Unconfined Compression ............... 50

CHAPTER 5: MATERIAL PARAMETER DETERMINATION ......................................52

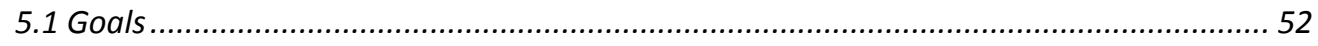

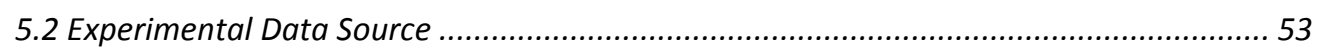

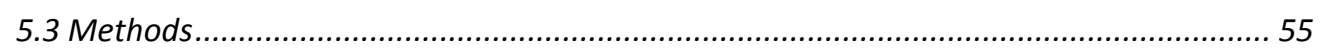

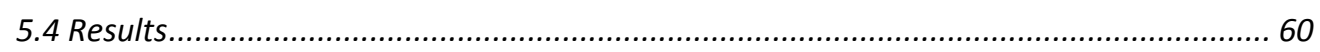




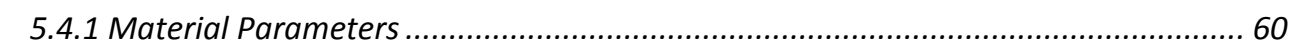

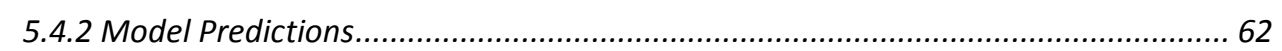

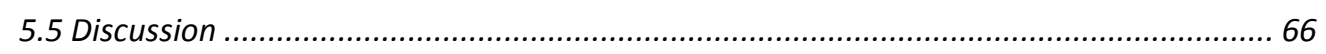

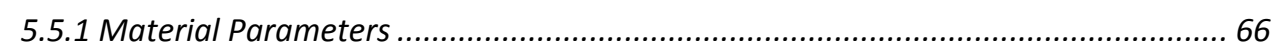





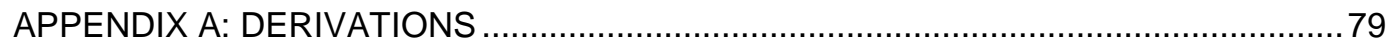

A.1.1 Derivative of The Determinant of A Second Order Tensor .................................................. 79

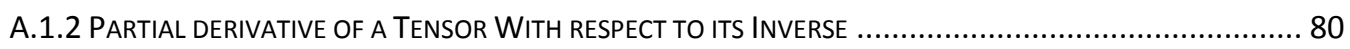

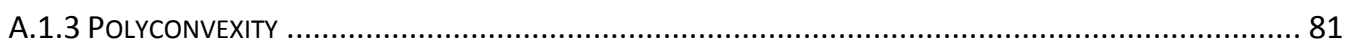

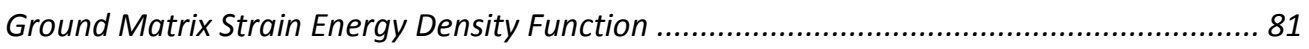

Collagen Strain Energy Density Function ................................................................... 82

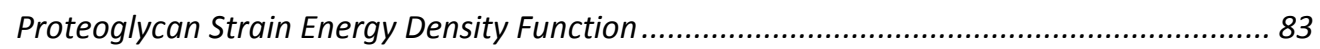



A.1.5 ConfINED AND UNCONFINED COMPRESSION CONSTITUENT STRESSES ......................................... 84

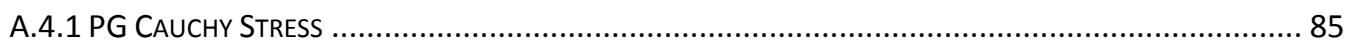





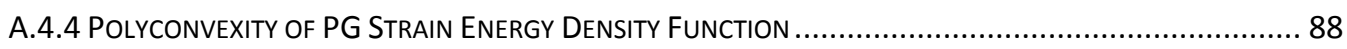

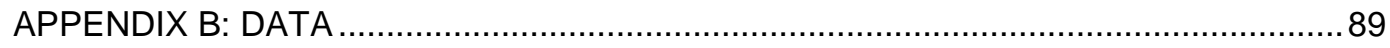





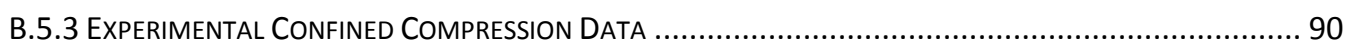

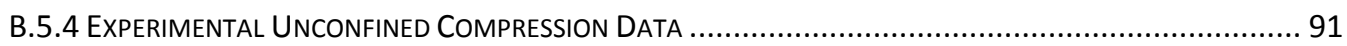

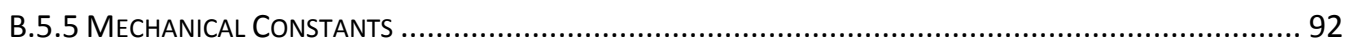

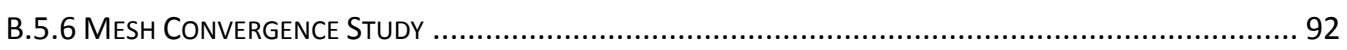




\section{List of Tables}

Figure

Page

Table 4.1. Biochemistry analysis results showing constituent biochemical measurements $\left(\mathrm{H}_{2} \mathrm{O}\right.$, GAG, and $\left.\mathrm{COL}\right)$.

Table 4.2. Results of curve fits to PB-cell model predictions.

Table 5.1. Material constants necessary for the constitutive FE model of AC with the appropriate units, and method of determination

Table 5.2. Complete objective function values 66

Table 5.3. Tissue source and determined True COL fiber modulus for D0 explants from this and previous studies.

Table B.1. Experimentally measured biochemical parameters.

Table B.2. Experimentally measured mean Tensile modulus, $\mathrm{E}^{+}$ 90

Table B.3. Experimentally measured mean $\mathrm{CC}$ modulus, $\mathrm{H}_{\mathrm{A}}$. 900

Table B.4. Experimentally measured mean UCC modulus, E - and Poisson's Ratio, $v$

Table B.5. Numerical values for mechanical constants true COL fiber modulus and MAT shear modulus. 


\section{List of Figures}

Figure

Page

Figure 2.1. UCC and CC testing configurations. 10

Figure 3.1. Elementary particle motion of a body B. 12

Figure 3.2. A stress-free reference configuration element, composed of PG, MAT and COL constituent elements.

Figure 3.3. Collagen fibril mapping and deformations between solid matrix configurations.

Figure 3.4. Schematic representation of a material point unit sphere showing a single pyramid element.

Figure 4.1. Compression and biochemical specimen preparation

Figure 4.2. Cartilage specimen showing rectangular and cylindrical coordinate systems.

Figure 4.3. Comparison of several curve fits to PB-cell model predictions

Figure 4.4. Constituent stress results for DO S layer and DO M Layer samples loaded in confined compression .

Figure 4.5. Constituent stress results for D0 S layer and D0 M Layer samples in unconfined compression

Figure 4.6. Correlation between COL/GAG mass ratio and predicted COL+ MAT constituent CC stress in confined compression. 
Figure 4.7. Correlation between COL/GAG mass ratio and predicted

COL+ MAT UCC constituent stress fin unconfined compression.

Figure 5.1. A patellofemorral groove showing harvest locations for "groove" and "ridge" specimens.

Figure 5.2. Initial constituent reference configurations and the corresponding initial stresses for PG, MAT and COL constituents

Figure 5.3. UCC and Tensile "box in a corner" FE boundary conditions.............58

Figure 5.4. True COL fiber modulus $E_{f} S$ and $M$ layer experimental groups .......61

Figure 5.5. Experimental results compared with theoretical model predictions for D0, S and M layers.

Figure 5.6. Experimental results compared with theoretical model predictions for D12 TGF- $\beta 1, \mathrm{~S}$ and M layers.

Figure 5.7. Experimental results compared with theoretical model predictions for D12 IGF-1, S and M layers.

Figure B.1. Mesh Convergence study for the D0 S layer group in tension and UCC 93

Figure B.2. Mesh convergence study results for theD0 S Layer group for initial stretch following the SM equilibrium step. 


\section{Chapter 1: Introduction}

Articular cartilage (AC) is a tissue that experiences a high level of biomechanical stress and provides a low friction, wear resistant surface in articulating joints (Williamson et al. 2003; Mow and Guo 2002). Traumatic injury, disease, and prolonged repetitive loading can lead to degradation, damage, and/or arthritis of AC. Arthritis is the leading cost of disability in the United States, affecting approximately 46 million people in the United States with the medical costs of treatment amounting to roughly 81 billion dollars in 2003 (Yelin et al. 2007). Intrinsic repair of $A C$ is ineffective likely due to the avascularity and low metabolic activity of the tissue (Buckwalter and Mankin 1998). Severe arthritis results in pain, stiffness, and decreased range of motion for patients. Presently, the only clinical option for treatment of severe arthritis is total joint replacement (Hochberg et al. 1995). Joint replacement surgery is invasive, expensive, and unlikely to completely restore joint function (Insall et al. 1976). Therefore, an effective means of treating arthritis or repairing damaged $A C$ tissue without resorting to total joint replacement is desired.

AC tissue engineering where tissue is modified and grown in vitro for use in vivo, presents a possible alternative to contemporary joint replacement treatments. Tissue engineered AC would replace locally damaged tissue or, for more extensive degradation or damage, the entire joint surface could be removed and replaced. AC replacement would not require the destruction of the joint structure as is inherent to joint replacement treatments. It may be necessary for a consistently successful AC tissue engineering strategy, to modify the 
geometry, mechanical properties, and tribological properties of an implant in order to match the requirements of the implant site and joint (Williams et al. 2009). Improving the understanding of structure function relationships of the constituents of $A C$ and the constitutive effects of growth on $A C$ is an important goal in current tissue engineering research.

$\mathrm{AC}$ has demonstrated complex mechanical properties during in vitro mechanical testing. For instance, $\mathrm{AC}$ has been shown to have highly non-linear equilibrium tension and compression properties where the tissue has a much higher Young's modulus in tension than in compression (Soltz and Ateshian 2000; Williamson et al. 2003; Huang C-Y et al. 2005; Ficklin et al. 2007). Additionally the mechanical properties, tissue structure, and tissue composition of $A C$ have been shown to vary with depth from the articular surface (Chen et al. 2001; Williams et al. 2010). AC is composed primarily of proteoglycan (PG) molecules and a collagen (COL) fibril network. Mechanically PG and COL have differential contributions to the overall AC mechanical response. In general, PG molecules resist compressive stress while the COL network resists shear and tensile stresses (Buschmann and Grodzinsky 1995). However, in situ measurement of constituent specific stresses is not possible.

Due largely to the complex mechanical properties of $\mathrm{AC}$, finite element analysis (FEA) modeling has emerged as a useful way of modeling the behavior of AC (Davol et al. 2008; Guilak and Mow 2000; Spilker et al. 1992). One method to ensure material stability for a FEA solution is polyconvexity. Polyconvexity ensures that at least one minimizing solution exists thereby improving FEA numerical convergence. The development of a FEA model also makes it possible to define the mechanical characteristics of individual constituents. Thereafter, 
each constituent is combined to form a complete tissue model that is derived directly from the true tissue composition and structure. In addition to reporting the overall SM response, a constitutive model of $A C$ could be used to vary the constituent properties to determine and tune the mechanical properties of $A C$ repair tissue. Also, a constitutive FEA model would allow individual constituent stresses and properties that cannot be experimentally measured to be predicted.

Early analytical models of $A C$ were incapable of accurately modeling the complex equilibrium, viscoelastic, and poroelastic behaviors of AC. Furthermore, some models lacked a direct connection to the actual constituents and/or structure of AC. Therefore, it was unlikely that insight into the structure-function relationships of $A C$ could be gained from such models. With recent advancements in regulated in vitro tissue growth (Williams et al. 2010; Williamson et al. 2003) including in vivo stimulation (Grimaud et al. 2002) there exists a need to better understand the constitutive functional changes and resulting mechanical manifestations of tissue engineered $A C$.

There are two primary aims of this work. The first aim is to develop a physically appropriate, polyconvex, continuum level, constitutive FE model of AC, and to use the model to determine stress equations and material constants for native $A C$ explants. The second aim is to use the FE model of $A C$ to determine how constitutive parameters change following in vitro culture. These objectives will help to develop more accurate analytical AC models, and elucidate the mechanical effects of in vitro culture on both the complete tissue and constituent level mechanical properties of $\mathrm{AC}$. 


\section{Chapter 2: Background Review}

\subsection{Proteoglycan Modeling}

The direct experimental measurement of in situ PG swelling stress ${ }^{1}$ is not possible through conventional experimental protocols. Experimental mechanical testing of $A C$ tissue reports only the solid matrix (SM) response, and it is not possible to isolate the specific mechanical response of the PG constituent from the complete SM response. Furthermore, Thomas et al. (2009) have proposed that PG-COL mechanical interactions which may influence the SM response exist and are remodeled during developmental growth. Although the mechanisms or precise mechanical effects of PG-Col interactions are not well understood, such PG-COL interactions may further complicate the direct measurement of in situ PG swelling stress. Therefore, development and experimental validation of PG models is limited to experimentation on PG solutions extracted from tissue (Basser et al. 1998; Buschmann and Grodzinsky 1995; Bathe et al. 2005).

The PG constituent is often modeled using electrochemical models (Eisenberg and Grodzinsky 1985; Lai et al. 1991; Buschmann and Grodzinsky 1995; Basser et al. 1998; Sun et al. 2004). While these models strive to develop PG models that are based on physically realistic continuum electromechanical interactions, they often require the determination of difficult to measure quantities such as fixed charge density, or glycosaminoglycan (GAG) molecule radius, and

\footnotetext{
${ }^{1}$ For the purposes of this study PG swelling stress and glycosaminoglycan osmotic pressure are considered to be the same.
} 
can result in overestimation of PG swelling stress and numerical instability in finite element analysis (FEA) (Klisch 2007; Davol et al. 2008; Buschmann and Grodzinsky 1995).

A continuum level model of the PG stress response can be combined with other constituent models (e.g. collagen fibrils) to create a complete tissue AC model. FEA allows the individual constituent responses to be isolated from the overall SM response. Additionally, continuum level models have been proven effective for modeling macroscopic mechanical responses without requiring extensive computational time necessary for micro level models (Buschmann and Grodzinsky 1995). A polyconvex continuum mechanics model of PG would be an appropriate and numerically stable means of modeling the macroscopic PG mechanical response in FEA modeling.

Studies have shown that the immature and mature AC equilibrium aggregate modulus $\left(\mathrm{H}_{\mathrm{A}}\right)$ and $\mathrm{AC}$ compressive modulus $(\mathrm{E})$, may initially decrease then increase with increasing compressive strains (Ficklin et al. 2001; Wang et al. 2003; Williams et al. 2010). Therefore, it is hypothesized that under large compressive strains (>15\% - 45\%) the COL fibers should undergo a transition from tension to compression. Current models often predict that the COL constituent would still be in tension even at $45 \%$ compressive strains (Oungoulian 2007). While the overestimation of PG swelling stress may still report a reasonable SM stress prediction, other constituents and in particular the COL constituent are affected through the stress balance hypothesis. Therefore, inaccuracies in PG stress predictions lead to inaccuracies in the COL fibril and other constituent stress predictions. With accurate constitutive predictions, it 
would be possible to determine the constituent specific mechanical responses to growth, in addition to the overall SM response.

\subsection{Collagen Fibril Modeling}

COL fibrils are thought to play an important role in the mechanics of many soft biologic tissues. In particular, for AC in mammals, type II collagen has been shown to be the predominant form of collagen fibrils in the extra cellular matrix (ECM) of cartilaginous tissues (Eyre 2001). Similar to in situ PG stress, the specific in situ constitutive response of the COL material presents a problem to researchers. Matrix depletion, wherein a portion of the PG constituent is digested to help isolate the COL mechanical response is possible. Asanbaeva et al. (2008) showed an increase in tensile modulus with matrix depletion for immature bovine $A C$ suggesting that in addition to $C O L$ fibrils, the PG constituent may play a role in the tensile behavior of AC. Furthermore, Thomas et al. (2009) have suggested that there may be COL-PG interactions that affect the mechanical SM response further complicating direct in situ COL response measurements. Studies have used MRI, TEM, and SEM ${ }^{2}$ imaging techniques to measure the orientation and elastic energy storage characteristics of the COL fibers (Eyre 2001; Clark 1990; Shinar et al. 2002) yet conclusive experimental stress-strain data for type II COL is not available.

\footnotetext{
${ }^{2} \mathrm{MRI}=$ magnetic resonance imaging; TEM = transmission electron microscopy; $\mathrm{SEM}=$ scanning electron microscopy
} 
Soft tissues containing COL are often modeled as fiber reinforced composite materials. An early model sought to capture the tensile and compressive nonlinearity of COL by defining a modulus for fibers in compression and a different modulus for fibers in tension (Schwartz et al. 1994). Different fiber models of COL have been implemented with varying complexity and degrees of fiber population and/or orientation (Julkunen et al. 2007; Fortin et al. 2000; Li et al. 2009). Recently, a novel approach to modeling a continuous distribution of collagen fibrils in soft tissues was proposed by Shirazi et al. (2010). Similar continuous fibrils models have been used by Gasser et al. (2006) to model arterial tissue, by Ateshian (2007) for generalized soft tissues, and by Ateshian et al. (2009) to model AC. Due to the complexity of fibril reinforced models, FEA is often implemented in order to reach a solution for a given boundary value problem. Therefore, it is essential that the selected COL fiber model be transferable to FEA theory for practical purposes.

Implementation of a COL material model requires the definition of one or more material constants, (e.g. fiber modulus) in order to define the mechanical characteristics of either the individual fibers, or a network of fibers. Strain independent (Soulhat et al. 1999), strain dependent (Korhonen et al. 2003; Lei and Szeri 2007), and strain dependent viscoelastic (Wilson et al. 2004), (Wilson et al. 2005) models for COL fiber modulus have been proposed previously. By fitting a fiber reinforced model to experimental data it is possible to vary the material constants, such as fiber modulus, in order to match the experimental results and thereby determine the material constants appropriate for a given experimental group. 
Previous studies (Korhonen et al. 2003; Wilson et al. 2004; Wilson et al. 2005; Lei and Szeri 2007) have varied COL fiber modulus to best fit experimental data for different experimental tissue sources. Wilson et al, (2005) predicted an equilibrium COL fiber modulus of $0.2737 \mathrm{MPa}$ at $10 \%$ tensile strain for Bovine AC. Presently, there is a wide discrepancy between $\mathrm{COL}$ fiber modulus predictions and experimentally measured COL fiber modulus; Silver et al. (2002) estimated an experimental COL fiber modulus of $7.0 \mathrm{GPa}$ in the surface region and 3.95 GPa in the deep region of mature human AC. This analytical and experimental discrepancy could be attributed to differences in the analytical models, experimental errors, and/or biological variation in the experimental groups used to validate and fit FE models.

\subsection{Ground Substance Matrix Modeling}

The ground substance matrix (MAT) material is intended to account for the mechanical response of other solid matrix components, including chondrocytes not already attributed to PG and COL constituents. Studies have shown that the ECM of $A C$ is composed primarily of $P G$ and $C O L$, however there are other molecules found in the ECM (Williamson et al. 2001; Kuettner 1992). Other studies suggest that these additional solid matrix components, as well as the chondrocytes, may contribute to the mechanical behavior of the complete tissue (Buschmann and Grodzinsky 1995; Chahine et al. 2005; Ehrlich et al. 1998). To account for the mechanics of the MAT material, an isotropic hyperelastic compressible Neo-Hookean material is proposed as a MAT material 
model. Previously, compressible Neo-Hookean material models have been used to model the mechanical behavior of isolated chondrocytes (Baaijens et al. 2005), and in other studies to account for the ground matrix of biological materials (Veress et al. 2002; Holzapfel 2006). Similarly to PG swelling stress, direct experimental measurement of in situ MAT stress is not possible.

\subsection{Mechanical Testing}

In order to accurately estimate constituent parameters, an experimental data set is required to compare model predictions to experimental measurements. For this study, previously obtained confined compression (CC), unconfined compression (UCC), and tensile data from Williams et al. (2010), and Stender et al.(2011) will be used. The protocols used for CC, UCC and tensile mechanical testing are summarized briefly as follows.

Bovine calf $A C$ explants were harvested from the patellofemoral groove (PFG) and prepared for mechanical testing. Samples were tested either sequentially in confined compression (CC) and unconfined compression (UCC) according to established protocols (Ficklin et al. 2007; Chen 2001 et al.) or uniaxial tension. For CC and UCC testing, disk shaped samples were prescribed a compressive displacement upon the top surface while force was simultaneously measured. For UCC testing (Figure 2.1, (a)), the equilibrium Young's modulus in the direction of the applied displacement E, and the Poisson's ratios $\mathrm{v}$, in the off axis directions were measured and recorded at $15 \%$ 
and $30 \%$ equilibrium compressive strains. Testing consisted of consecutive $400 \mathrm{~s}$ ramps to $15 \%$ and then $30 \%$ CC or UCC strain. After reaching the desired strain level, stress relaxation to equilibrium was defined as a change in stress of $<0.003$ MPa over $180 \mathrm{~s}$. The tissue samples were kept submerged in a phosphate buffered saline solution with protease inhibitors $(\mathrm{PBS}+\mathrm{PI})$ throughout the testing.

For CC testing impermeable top and bottom platens were used to apply the fixed displacement to the tissue (Figure 2.1, (b)) During CC testing, the equilibrium confined compression modulus, $\mathrm{H}_{\mathrm{A}}$, was measured and recorded at $15 \%$ and $30 \%$ strains. Tissue samples were constrained radially within an impermeable confining chamber. Porous platens constrained the top and bottom surfaces. The entire testing apparatus (platens, tissue sample, and confining chamber) were submerged in PBS+PI for the duration of the test.



Figure 2.1. Diagram showing (a) UCC and (b) $C C$ testing configurations for cylindrical AC samples. For UCC Young's Modulus, E and Poisson's ratios in the off axis directions were determined. For $\mathrm{CC}$ the confined compression modulus $\boldsymbol{H}_{A}$ was obtained.

Tensile specimens were held between two clamps and stretched to the desired $5 \%$ and $10 \%$ tensile strains. Tensile specimens were tested similarly to established protocols (Asanbaeva et al. 2008; Williamson et al.2003) only with relaxation time increased to 5000 seconds to more accurately determine 
equilibrium properties. A curve fit to the resulting stress relaxation curve was used to estimate the equilibrium force which was used to calculate the equilibrium Young's modulus. A PBS+PI solution was pumped over the tissue throughout the duration of the testing and relaxation to maintain hydration. 


\section{Chapter 3: Theory}

The following descriptions are intended to provide an outline of the theory relevant and necessary to the understanding of this study. Additional derivations and complete descriptions can be found in the appendices and the associated references.

\subsection{Kinematics}

Consider a body that initially, at time $t_{0}=0$, occupies a reference configuration $k_{0}(B)$. At a later time $t$, the same body occupies the current configuration $k(B)$, (Figure 3.1).

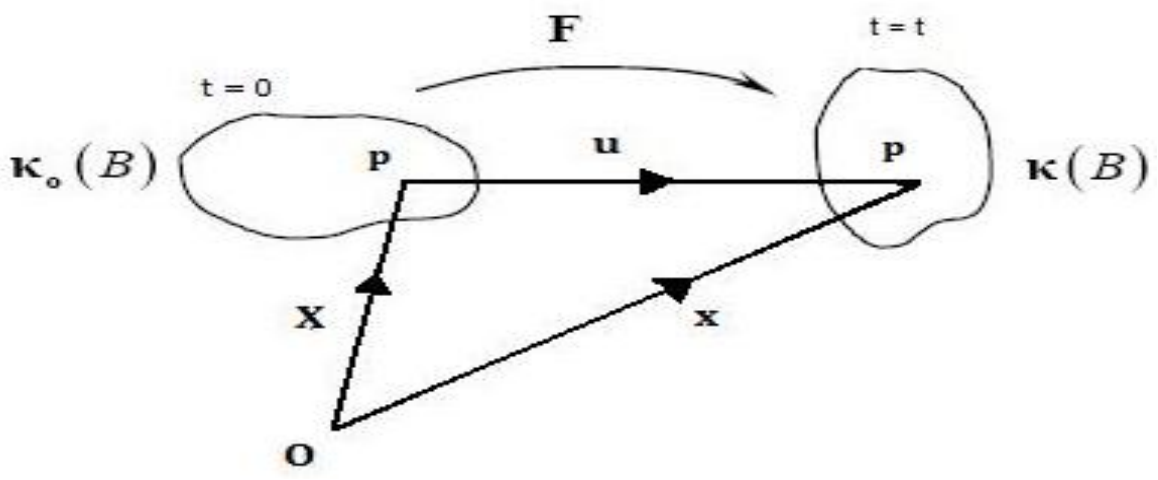

Figure 3.1. Elementary particle motion of a body $B$. The vectors $\mathrm{X}$ and $\mathrm{x}$ track the position of point $p$ in the reference and current configurations respectively, in reference to the origin $\mathrm{O}$. 
The displacement of a material point can be defined by the displacement vector, (u).

$$
\mathbf{u}=\mathbf{x}-\mathbf{x}
$$

The displacement increment gradient tensor $(\mathbf{L})$, is defined as

$$
\mathrm{L}=\frac{\partial \delta \mathrm{u}}{\partial \mathrm{x}}
$$

where $\delta \mathbf{u}$ is an increment of displacement. The rate of deformation tensor (D), represents the symmetric portion of the displacement increment gradient tensor (L), and is defined in indicial notation as

$$
\mathrm{D}_{\mathrm{ij}}=\frac{1}{2}\left(\mathrm{~L}_{\mathrm{ij}}+\mathrm{L}_{\mathrm{ji}}\right)
$$

The skew portion of the rate of deformation tensor (D), is referred to as the spin tensor, $(\omega)$ and is defined using indicial notation in Equation 3.4.

$$
\omega_{\mathrm{ij}}=\frac{1}{2}\left(\mathrm{~L}_{\mathrm{ij}}-\mathrm{L}_{\mathrm{ji}}\right)
$$

A unique material point $\mathrm{p}$, on the body $\mathrm{B}$, in the reference configuration $k_{0}$, has a position $\mathrm{X}$, and at a later time $t$, in the current configuration $k$ has a position $\mathbf{x}$. The invertible motion of the body $\mathrm{B}$ between $k_{0}$ and $k$ can be described by the mapping

$$
\mathbf{x}=\chi(\mathbf{X}, t)
$$

with the deformation gradient tensor defined as 


$$
\mathbf{F}=\frac{\partial \chi(\mathbf{X}, t)}{\partial \mathbf{X}}
$$

Volumetric changes in the deformable compressible body B are described by the determinant of $\mathbf{F}$, which is referred to as the Jacobian and is defined below

$$
\mathrm{I}=\operatorname{det}(\mathbf{F})
$$

The law of conservation of mass asserts that mass of a body is constant under motion and constant in every configuration. The continuity equation is derived by applying conservation of mass across the reference configurations (Mase et al. 2009). The resulting continuity constraint is shown below in the Lagrangian form.

$$
\rho \mathrm{J}=\rho_{0}
$$

where $\rho$ and $\rho_{0}$ are the density in the current and reference configurations, respectively and I is the Jacobian.

By applying the polar decomposition theorem, the deformation gradient $\mathbf{F}$ is expressed as follows:

$$
\begin{aligned}
& \mathbf{F}=\mathbf{R U} \\
& \mathbf{F}=\mathbf{V R}
\end{aligned}
$$

where $\mathbf{R}$ is the rotation tensor and $\mathbf{U}$ and $\mathbf{V}$ are the right and left stretch tensors, respectively. Note that the rotation tensor, $\mathbf{R}$ is proper orthogonal and that both $\mathbf{U}$ and $\mathbf{V}$ are symmetric positive definite. The right $(\mathbf{C})$ and left $(\mathbf{B})$ Cauchy-Green deformation tensors are related to the respective stretch tensors, and the deformation gradient through the following relationships: 


$$
\begin{aligned}
& \mathbf{C}=\mathbf{U}^{2}=\mathbf{F}^{\mathrm{T}} \mathbf{F} \\
& \mathbf{B}=\mathbf{V}^{2}=\mathbf{F F}^{\mathbf{T}}
\end{aligned}
$$

$\mathbf{C}$ and $\mathbf{B}$ are consequently positive definite. The Lagrangian strain tensor $(\mathbf{E})$, is used to evaluate how much a given deformation differs locally from rigid body motion (Lubliner 2008). The Lagrangian strain tensor, $(\mathbf{E})$ is defined as follows

$$
\mathbf{E}=\frac{1}{2}(\mathrm{C}-\mathrm{I})
$$

where $\mathbf{C}$ is the right Cauchy-Green deformation tensor and $\mathbf{I}$ is the identity tensor.

\subsection{Stress}

The strain energy density function of a Green-elastic material is a scalar valued function that relates the strain energy density of a material to the deformation gradient, or a corresponding measure of deformation.

$$
W=\bar{W}(\mathbf{F})=\widetilde{W}(\mathbf{C})
$$

Note that the strain energy function is intrinsic to a particular material, and may include relevant material constants.

For a hyperelastic, or a Green-elastic material, the stress-strain relationship is derived from the strain energy function as shown below.

$$
\mathbf{S}=2 \frac{\partial W}{\partial \mathbf{c}}
$$


where $\mathbf{S}$ is the second Piola-Kirchoff stress tensor. The Kirchoff stress measure (ז), Cauchy stress tensor $(\mathbf{T})$, and the first Piola-Kirchoff stress tensor $(\mathbf{P})$, are obtained from the Second Piola-Kirchoff stress using the following transformations.

$$
\mathbf{\tau}=J \mathbf{T}=\mathbf{F S F}^{\mathrm{T}}=\mathbf{P F}^{\mathrm{T}}
$$

The Cauchy stress tensor $(\mathbf{T})$, is current configuration force normalized to current configuration area, or true stress. The first Piola-Kirchoff stress tensor $(\mathbf{P})$, is current configuration force divided by reference configuration area, or engineering stress. The Kirchhoff stress measure (ז), has no obvious physical significance, but is useful for finite strain problems for total form constitutive equations because the Kirchoff stress measure is the gradient of displacement variation. For the purposes of this study, variation in work is done by a variation in the Kirchoff stress measure and a variation in stretch.

Similarly to the derivation of the Second Piola Kirchoff Stress tensor, the elasticity tensor can be derived using the following relationship.

$$
\mathbb{C}=2 \frac{\partial s}{\partial \mathrm{C}}
$$




\subsection{Solid Matrix Constituents}

For the purposes of this study the solid matrix (SM) of AC is defined to be composed of PG, COL and MAT materials each with initial configurations $k_{0}^{\mathrm{PG}}$, $k_{0}^{\mathrm{COL}}$, and $k_{0}^{\mathrm{MAT}}$ respectively (Figure 3.2).

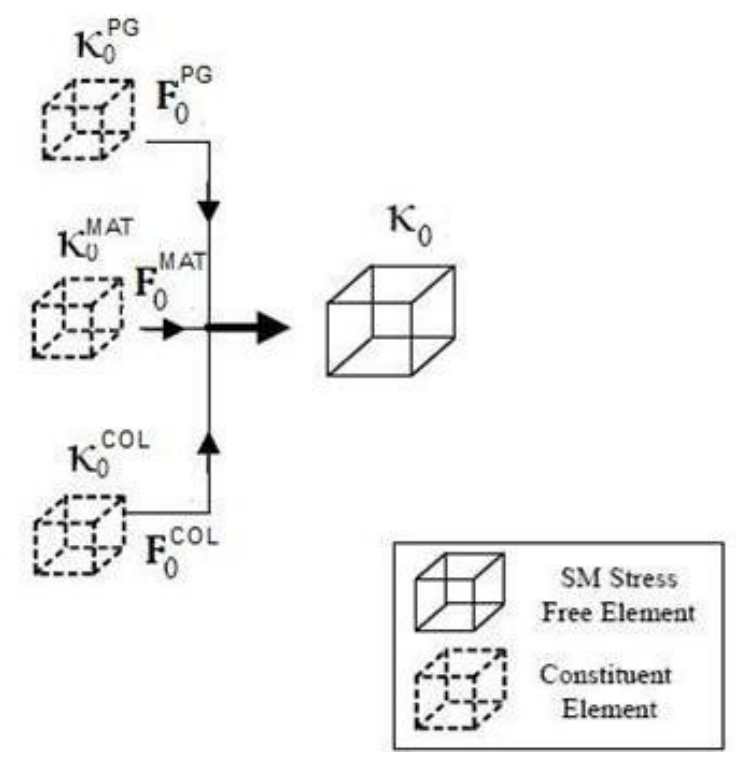

Figure 3.2. The SM stress-free reference configuration element, $\boldsymbol{k}_{\mathrm{R}}$ is composed of $\mathrm{PG}$, MAT and COL constituent elements. Each constituent element undergoes an initial deformation to reach the stress-free equilibrium condition.

Note that due to the intrinsic spherical swelling stress in the PG constituent reference configuration $k_{0}^{\mathrm{PG}}$ all constituents undergo initial deformations to meet the initial stress-free SM element condition. These initial deformations lead to a tensile pre-stress in the COL and MAT constituents in the SM reference configuration (Klisch et al. 2008; Thomas et al. 2009). 


\subsubsection{Immobility Constraint}

This study uses the immobility constraint to determine the constitutive deformation gradient tensors relative to the solid matrix deformation gradient tensor. The immobility constraint assumes that PG, COL, and MAT molecules are bound to the SM, and therefore each constituent's total deformation gradient tensor $\left(\mathbf{F}^{\mathrm{PG}}{ }_{\text {, }} \mathrm{F}^{\mathrm{COL}}\right.$, and $\left.\mathbf{F}^{\mathrm{MAT}}\right)$ is equal to the $\mathrm{SM}$ deformation gradient tensor $\mathbf{F}^{S M}$ relative to the SM reference configuration..

$$
\mathbf{F}^{\mathrm{PG}}=\mathbf{F}^{\mathrm{COL}}=\mathrm{F}^{\mathrm{MAT}}=\mathrm{F}^{\mathrm{SM}}
$$

Note that relative to the respective constituent reference configurations (as opposed to the SM reference configuration), each constituent can have a deformation gradient tensor that is different from other constituents. The immobility constraint has not been conclusively verified, however several studies have successfully implemented the immobility constraint in AC modeling (Klisch et al. 2008; Ficklin et al. 2009; Oungoulian 2007). The immobility constraint is a physically reasonable and necessary assumption for constitutive modeling of AC.

\subsubsection{Stress Balance Laws}

The stress balance hypothesis is used in order to quantify the mechanical response of the SM which is composed of multiple constituents. The stress 
balance hypothesis is a common assumption in continuum mixture theory (Atkin and Craine 1976). The stress balance hypothesis states that the SM stress is equal to the sum of each of the constitutive stresses shown below for the Cauchy stress tensor, in Equation 3.19.

$$
\mathbf{T}^{\mathrm{SM}}=\mathbf{T}^{\mathrm{PG}}+\mathbf{T}^{\mathrm{COL}}+\mathbf{T}^{\mathrm{MAT}}
$$

Note that the stress balance hypothesis allows non-zero constituent stresses in the stress free SM reference configuration.

\subsection{Abaqus Implementation}

Implementation of the AC material developed in this project into Abaqus (SIMULIA Providence, RI v6.7), an FEA solver, requires the development of a user material (UMAT). Abaqus requires that the UMAT define a Cauchy stress equation, and a material Jacobian matrix for the given UMAT. Due to the constitutive nature of the AC model, and to make code development easier, Cauchy stress and constituent material Jacobian matrices are defined for each constituent individually and then summed following the stress balance laws to report SM results. 


\subsubsection{Material Jacobian Matrices}

A material Jacobian matrix is used during the iterative solution process to direct the progression of the solution for each sequential iteration towards the final solution. The material Jacobian matrix, $\mathbb{C}^{\text {Iac }}$ must be defined in the UMAT and is calculated, along with Cauchy stress by the FE solver for each iteration at each material point.

The elasticity tensor is generally defined as the partial derivative of a stress increment with respect to a strain increment.

$$
\mathbb{C}=\frac{\partial \Delta \sigma}{\partial \Delta \varepsilon}
$$

The definition of the elasticity tensor shown in equation 3.20 applies to material models with small deformations and/or small volumetric changes. For large volumetric changes and geometric nonlinearity, Abaqus requires that a special form of the stiffness matrix, called the exact consistent Jacobian matrix (ECJM) (SIMULIA 2007) as defined below.

$$
\mathbb{C}^{\mathrm{ECJM}}=\frac{1}{\mathrm{~J}} \frac{\partial \Delta(\mathrm{JT})}{\partial \Delta \varepsilon}
$$

Note that the JT term is equivalent to the Kirchoff stress measure. Within the FEA the ECJM is equivalent to a material stiffness matrix.

Due to the complex material model developed for AC, an alternative method of calculating the material Jacobian matrix is used. The material 
Jacobian matrix ( $\mathbb{\mathbb { U }}^{\mathrm{aac}}$ ) for total form constitutive equations is defined as follows by Abaqus (SIMULIA 2007).

$$
\delta I(\tau)=J \mathbb{C}^{\top a c} ; \delta D
$$

where $\delta^{I}(\tau)$ is the Jaumann rate of the Kirchoff stress, shown below and $\delta \mathbf{D}$ is the increment of the gradient of displacement variation with respect to current position. Equation 3.22 will be used to define the material Jacobian matrix that combined with a Cauchy stress equation will completely define material behavior of the AC material model in the FE solver.

This study will use the Jaumann stress rate which is invariant under rigid body rotation, and thus preferred for constitutive equations. Although other stress rates are appropriate, the Jaumann stress rate is commonly used in computational analysis because it is relatively easy to implement. The Kirchoff stress rate, $(\delta \mathrm{I}(\boldsymbol{\tau}))$ is defined as.

$$
\delta I(\tau)=\delta(\tau)-(\delta \omega \tau-\tau \delta \omega)
$$

Note that the Jaumann rate of the Kirchoff stress, $(\delta I(\tau))$ and the total increment of Kirchoff stress, $(\delta(\mathbf{\tau}))$ are different. However, the total increment of Kirchoff stress must be known to calculate the Jaumann rate of Kirchoff stress and consequently, the Jacobian stiffness matrix necessary for Abaqus implementation. 
The total increment of Kirchoff stress is defined in indicial notation as shown below. ${ }^{3}$

$$
\delta \tau_{i j}=\delta F_{i K} S_{K H} F_{j H}+F_{i K} S_{K H} \delta F_{j H}+F_{i K} \delta S_{K H} F_{j H}
$$

The $\delta \mathrm{F}_{\mathrm{ij}}$ terms can be expanded using the displacement increment tensor, $(\mathbf{L})$ defined previously in equation 3.2.

$$
\delta \mathrm{F}_{\mathrm{iA}}=\delta \mathrm{L}_{\mathrm{ij}} \mathrm{F}_{\mathrm{jA}}
$$

The $\delta \mathrm{S}_{\mathrm{KH}}$ term requires the calculation of the fourth order elasticity tensor shown in equation 3.17 as well as the deformation gradient tensor $(\mathbf{F})$, and the rate of deformation tensor, (D) from Section 3.1

$$
\delta \mathrm{S}_{\mathrm{AB}}=\widetilde{\mathbb{C}}_{\mathrm{ABCD}} \mathrm{F}_{\mathrm{kC}} \delta \mathrm{D}_{\mathrm{kl}} \mathrm{F}_{\mathrm{lD}}
$$

Note that this elasticity tensor $\left(\widetilde{\mathbb{C}}_{\mathrm{ABCD}}\right)$ is different than the material Jacobian stiffness matrix ( $\left.\mathbb{\mathbb { U }}^{\mathrm{lac}}\right)$. Using equation 3.25 and equation 3.26 the variation in Kirchoff stress shown in equation 3.24 can be rewritten as shown below.

$$
\delta \tau_{i j}=L_{i B} \tau_{B j}+L_{i A} \tau_{A j}+\widetilde{\mathbb{C}}_{A B C D} F_{i A} F_{j B} F_{k C} F_{l D} \delta D_{k l}
$$

which is rearranged to form the equation shown below

$$
\begin{aligned}
& \delta \tau_{i j}=\left[\frac{1}{2}\left(\delta_{i k} \delta_{p r}+\delta_{p k} \delta_{i r}\right) \tau_{p j}+\frac{1}{2}\left(\delta_{j k} \delta_{n r}+\delta_{n k} \delta_{j r}\right) \tau_{i n}+\widetilde{C}_{\mathrm{ABCD}} F_{i A} F_{j B} F_{k C} F_{r D}\right] \delta D_{k r}+ \\
& \delta \omega_{i p} \tau_{p j}-\tau_{i n} \delta \omega_{n j}
\end{aligned}
$$

\footnotetext{
${ }^{3}$ The following theory developed to define the material jacobian matrix was developed by Psquale Vena and Reza Shirazi.
} 
Substituting into equation 3.23 and canceling terms, gives the Jaumann rate of the Kirchoff stress as shown below

$$
\begin{gathered}
\delta I\left(\tau_{\mathrm{ij}}\right)=\left[\left[\frac{1}{2}\left(\delta_{\mathrm{ik}} \delta_{\mathrm{pr}}+\delta_{\mathrm{pk}} \delta_{\mathrm{ir}}\right) \tau_{\mathrm{pj}}+\frac{1}{2}\left(\delta_{\mathrm{jk}} \delta_{\mathrm{nr}}+\delta_{\mathrm{nk}} \delta_{\mathrm{jr}}\right) \tau_{\mathrm{in}}\right]\right. \\
\left.+\left[\widetilde{\mathbb{C}}_{\mathrm{ABCD}} \mathrm{F}_{\mathrm{iA}} \mathrm{F}_{\mathrm{jB}} \mathrm{F}_{\mathrm{kCC}} \mathrm{F}_{\mathrm{rD}}\right]\right] \delta \mathrm{D}_{\mathrm{kr}}
\end{gathered}
$$

The Jaumann rate of the Kirchoff stress is substituted into equation 3.22 to give the Jacobian stiffness matrix used in Abaqus implementation.

$$
\widetilde{\mathbb{C}}_{\mathrm{ijkr}}^{\mathrm{Jac}}=\frac{1}{\mathrm{~J}}\left[\left[\frac{\mathrm{J}}{2}\left(\delta_{\mathrm{ik}} \delta_{\mathrm{pr}}+\delta_{\mathrm{pk}} \delta_{\mathrm{ir}}\right) \mathrm{T}_{\mathrm{pj}}+\frac{\mathrm{J}}{2}\left(\delta_{\mathrm{jk}} \delta_{\mathrm{nr}}+\delta_{\mathrm{nk}} \delta_{\mathrm{jr}}\right) \mathrm{T}_{\mathrm{in}}\right]+\left[\widetilde{\mathbb{C}}_{\mathrm{ABCD}} \mathrm{F}_{\mathrm{iA}} \mathrm{F}_{\mathrm{jB}} \mathrm{F}_{\mathrm{kC}} \mathrm{F}_{\mathrm{rD}}\right]\right]
$$

Note that the Cauchy stress which is already necessary for Abaqus implementation is used in place of Kirchoff stress in the definition of the material Jacobian matrix to simplify UMAT development.

\subsubsection{Polyconvexity}

The general theorem of polyconvexity, which is developed from the principles of variational calculus, states that if the strain energy function, $W$ is polyconvex, then $W$ is elliptic for all deformations. Therefore a polyconvex strain energy function guarantees that there exists at least one minimizing deformation 
(Pedregal 1987). Polyconvexity does not guarantee a unique solution. However, polyconvexity does guarantee that incremental stability will not be lost for a given strain energy function. Because of the incremental nature of FE problems incremental stability is sufficient for the solution of most problems. Because polyconvexity does not guarantee a unique solution, it is important to validate a FE solution to ensure that the desired strain energy state is reached.

\subsection{Constituent Material Models}

\subsubsection{Proteoglycan Swelling Stress Models}

\section{Ideal Donnan Model}

A macroscopic theory where the electrostatic repulsion contribution from the fixed charge density (FCD) of the ECM of AC has been viewed as a Donnan osmotic swelling pressure as proposed by Maroudas (1979). The Donnan model (Overbeek 1956) requires no assumption of molecular structure. In the Donnan model, the constant electrostatic potential of a polyelectrolyte phase is determined by the FCD and results in the osmotic swelling pressure of the PG. The Donnan model is shown below.

$$
\pi^{P G}=2 R T C_{0}\left(\gamma \sqrt{\frac{\rho_{m}^{2}}{4 F^{2} C_{0}^{2}}+1}-\varphi\right)
$$


Where $F$ is the Faraday constant (coul/mol), $R$ is the universal gas constant $(\mathrm{kJ} / \mathrm{K}-\mathrm{mol}), T$ is temperature $(\mathrm{K}), C_{0}$ is the external ion concentration $(\mathrm{M}), \rho_{\mathrm{m}}^{2}$ is the FCD (coul/L-fluid), $\gamma$ is the external osmotic coefficient, and $\varphi$ is the internal osmotic coefficient. The ideal assumption for the Donnan model osmotic coefficients is shown below:

$$
\gamma=\varphi=1
$$

with the ideal assumption, the Donnan model reduces to the ideal Donnan model as shown below:

$$
\pi^{P G}=2 R T C_{0}\left(\sqrt{\frac{\rho_{m}^{2}}{4 F^{2} C_{0}^{2}}+1}-1\right)
$$

\section{Modified Donnan}

A study, (Buschmann and Grodzinsky 1995) proposed that external ions present in physiologic saline, shield charge within the tissue. This ionic shielding would lead to a decrease in osmotic pressure with an increase in external ion concentration. The ideal model neglects this external ionic shielding and consequently has been shown to over predict PG swelling stress for low FCD (Buschmann and Grodzinsky 1995; Oungoulian et al. 2007). Furthermore, the Donnan model is unable to capture the highly nonlinear pressure vs. PG concentration behavior (Buschmann and Grodzinsky 1995). For constitutive models, over prediction of PG swelling stress can lead to incorrect constituent responses for all constituents, and possibly incorrect SM response predictions.

A modified Donnan model that accounts for external ionic shielding has been used by Buschmann et al. (1995). Values for the external osmotic 
coefficient, $\gamma$, were determined based on external solution FCD, and values for the internal osmotic coefficient, $\varphi$, were determined based on a PG associated FCD. The modified Donnan model improves the predictions of the ideal Donnan model, particularly for low FCD.

\section{Poisson-Boltzmann}

The Poisson-Boltzmann (PB) model is based on a microstructural, molecular level solution to the PB-cell relationship in equation 3.34 for a unit cell containing a charged GAG molecule and a surrounding atmosphere of mobile ions. The PB-unit cell model accounts for the space-varying electrical potential of GAG molecules within the PG structure. Therefore, a fundamental difference between the PB cell and Donnan models is the characteristic length scale. In the Donnan model, each continuum element contains many macromolecules. The length scale in the PB model is inherently smaller $(\sim 1.0 \mathrm{~nm})$ (Figure 3.3).

The Poisson-Boltzmann equation which gives electrostatic potential as a function of radius, $(R)$ within the unit cell is shown below.

$$
\nabla^{2} \Phi=\frac{2 F C_{0}}{E_{f}} \sinh \left(\frac{F \Phi}{R T}\right)
$$

Where $\nabla^{2}$ is the Laplace operator, $F$ is the Faraday constant (coul/mol), $R$ is the universal gas constant $(\mathrm{kJ} / \mathrm{K}-\mathrm{mol}), T$ is temperature $(\mathrm{K}), C_{0}$ is the external ion concentration $(M), \epsilon_{f}$ is the permittivity in the fluid phase of the unit cell $\left(\mathrm{coul} / \mathrm{m}^{2}\right)$, and $\Phi$ is electrostatic potential (kJ/coul). For a unit cell composed of a charged GAG molecule in an aqueous solution equation 3.34 is subject to the following boundary conditions: 


$$
\sigma=-\left.\epsilon_{f} \frac{d \Phi}{d r}\right|_{r=\alpha} \quad \text { and } \quad 0=\left.\frac{d \Phi}{d r}\right|_{r=R}
$$

where $a$ is the charged PG molecule radius, and $R$ is the unit cell radius. The microcontinuum osmotic pressure difference given by the PB cell model is shown below:

$$
\pi_{P B}^{P G}=2 R T C_{0}\left(\cosh \left(\frac{\mathrm{F} \Phi(\mathrm{R})}{R T}\right)=1\right)
$$

where $R$ is the universal gas constant $(\mathrm{kJ} / \mathrm{K}-\mathrm{mol}), T$ is temperature $(\mathrm{K}), C_{0}$ is the external ion concentration (M), F is Faraday's constant (coul/mol), and $\Phi(R)$ is the microcontinuum electrostatic charge potential $(\mathrm{kJ} / \mathrm{coul})$ determined from the solution to equation 3.34 with the boundary conditions in 3.35. Unit cell radius, $(\mathrm{R})$ is approximated from PG concentration through the following relationship:

$$
R=\sqrt{\pi 2 b N \frac{C_{P G}}{M_{C S}}}
$$

Where $b$ is GAG interchange distance $(\mathrm{m}), \mathrm{N}$ is Avogadro's number (atoms $/ \mathrm{mol}$ ), $M_{C S}$ is the molecular weight of a dissociated chondroitin sulfate disaccharide ( $\mathrm{g} / \mathrm{mol})$, and $C_{P G}$ is the $P G$ concentration $\left(\mathrm{g} / \mathrm{m}^{3}\right)$.

An exact solution to the PB equation is unavailable. The PB-cell equation is solved subject to the boundary conditions in equation 3.35 numerically. Presently the solution to the PB-cell model provides the most accurate PG swelling stress predictions compared to experimental results, particularly for low FCD. Furthermore, the PB-cell model more accurately predicts equilibrium CC modulus for AC compared with the Donnan model (Buschmann and Grodzinsky 1995). 


\subsubsection{Collagen Material Model}

The COL material model used in this project was developed by Shirazi et al. (2010). A brief description and relevant results will be reproduced here for reference. For a complete description refer to (Shirazi et al. 2010).

Initially, the COL material undergoes an initial tensile pre-strain deformation $\left(\mathrm{F}_{0}^{\mathrm{COL}}\right)$ to reach a stress free $\mathrm{SM}$ reference configuration, $\mathrm{k}_{0}$. The $\mathrm{SM}$, and by the immobility constraint, the COL material then undergo an overall SM deformation (F) (Figure 3.4).



Figure 3.3. The deformation gradient tensor $\mathrm{F}_{0}^{\mathrm{COL}}$ maps the $\mathrm{COL}$ material from the initial stress-free COL configuration, $\boldsymbol{k}_{0}^{C O L}$ to the stress-free SM configuration. An overall SM deformation gradient Tensor, F maps the SM to the current configuration $\boldsymbol{k}$. 
Note that in order to reach the SM stress free reference configuration, an initial tensile pre-strain deformation $\left(\mathrm{F}_{0}^{\mathrm{COL}}\right)$, of the $\mathrm{COL}$ fibril network is necessary. This initial deformation modifies the total COL deformation gradient $\left(\mathbf{F}^{\mathrm{COL}}\right)$, and consequently, the right Cauchy Green deformation tensor $(\mathbf{C})$ as shown below:

$$
\begin{gathered}
\mathbf{F}^{\mathrm{COL}}=\mathrm{FF}_{0}^{\mathrm{COL}} \\
\mathbf{C}^{\mathrm{COL}}=\left(\mathbf{F}^{\mathrm{COL}}\right)^{\mathrm{T}} \mathbf{F}^{\mathrm{COL}}=\left(\mathbf{F}_{0}^{\mathrm{COL}}\right)^{\mathrm{T}} \mathbf{C F}_{0}^{\mathrm{COL}}
\end{gathered}
$$

Therefore, the COL network Lagrangian strain tensor in direction $\mathbf{n}$ can be defined as follows

$$
\mathbf{E}_{\mathbf{n}}^{\mathrm{COL}}=\mathbf{n} \cdot\left[\left[\left(\left(\mathbf{F}_{0}^{\mathrm{COL}}\right)^{\mathrm{T}} \mathbf{C} \mathbf{F}_{0}^{\mathrm{COL}}-\mathbf{I}\right)\right] \mathbf{n}\right]
$$

Where $(v)$ is the scalar dot product operator.

Shirazi et al. (2010) have proposed a method of defining a COL fibril volume fraction at all material points in the material model. The COL volume fraction $\left(\Phi_{\mathrm{COL}}\right)$, is taken into account when developing a complete strain energy density function for a material where other constituents (e.g. GAG and MAT) also make up the volume fraction at a given material point (i.e. $\left.\Phi_{\mathrm{COL}}<1\right)$

$$
\Phi^{\mathrm{COL}}=\frac{\mathrm{v}_{\text {fibers }}}{\mathrm{v}_{\text {total }}}
$$

In the model proposed by Shirazi et al. (2010), a local spherical coordinate system is assumed at each material point where a local unit sphere is divided into pyramid elements each with volume dV. Each pyramid element contains a volume fraction of $\mathrm{COL}$ fibers $\left(\mathrm{dV}^{\mathrm{f}}\right)$ (Figure 3.4$)$. 


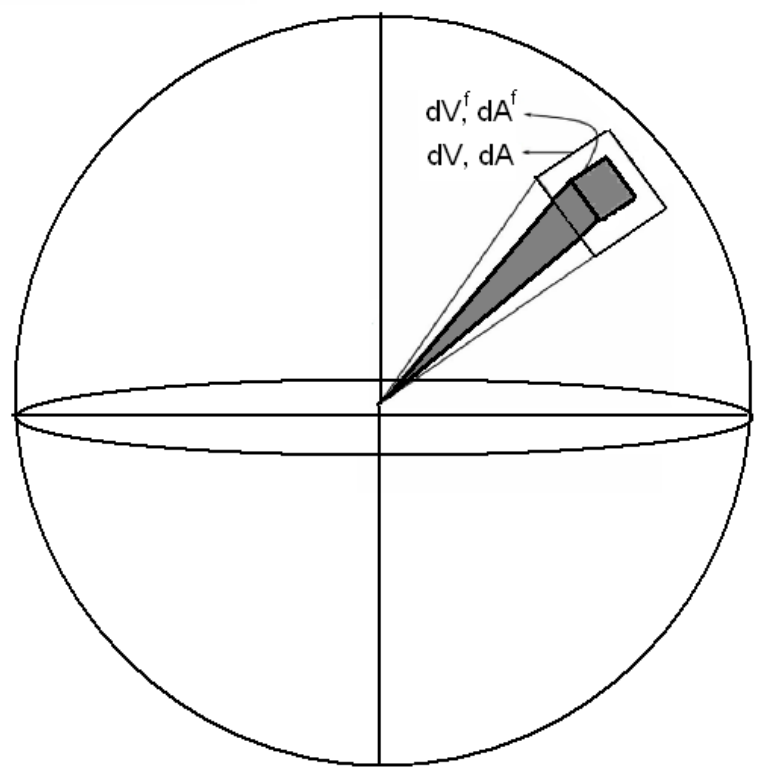

Figure 3.4. Schematic representation of a material point unit sphere showing a single pyramid element. The gray volume of the elemental pyramid represents the volume $\mathrm{dV}^{\dagger}$ occupied by COL fibers inside the total pyramid volume $\mathrm{dV}$.

To account for a continuous distribution of fibers with either an isotropic or an anisotropic fibril distribution, a fibril volume fraction distribution, $R(\theta, \Phi)$ function (in spherical coordinates) is defined.

$$
R(\theta, \Phi)=\frac{\Phi_{\mathrm{n}}^{\mathrm{COL}}}{\mathrm{v}_{\text {total }}}
$$

To determine the total COL volume fraction at a material point, for fibers in each direction $\mathbf{n}$, the fibril volume distribution function is integrated over volume of the unit sphere.

$$
\int_{\theta=0}^{\pi} \int_{\varphi=0}^{2 \pi} R(\theta, \Phi) \frac{1}{3} \sin \theta \mathrm{d} \theta \mathrm{d} \varphi=\Phi_{\text {total }}^{\mathrm{CoL}}
$$


It is hypothesized that COL fibers do not support compressive stress (i.e. when $\mathbf{E}_{\mathbf{n}}<0$ ). Therefore, the unitless Heaviside step function is introduced as defined below.

$$
H\left(\mathbf{E}_{\mathbf{n}}\right)= \begin{cases}0, & \mathbf{E}_{\mathbf{n}}<0 \\ 1, & \mathbf{E}_{\mathbf{n}} \geq 0\end{cases}
$$

with the inclusion of the Heaviside step function and the fibril volume distribution functions it is now possible to define the COL fibril strain energy function ( $\left.W^{\mathrm{COL}}\right)$, in spherical coordinates as shown below.

$$
W^{\mathrm{COL}}=\int_{\theta=0}^{\pi} \int_{\varphi=0}^{2 \pi} \frac{1}{3} \mathrm{R}(\theta, \Phi) \mathrm{H}\left(\mathbf{E}_{\mathbf{n}}\right) \Psi\left(\mathbf{E}_{\mathbf{n}}\right) \sin \theta \mathrm{d} \theta \mathrm{d} \varphi
$$

where $\Psi\left(\mathbf{E}_{\mathbf{n}}\right)$ is the pure COL strain energy density function as a function of Lagrangian strain. Using this COL fibril strain energy function, the second PiolaKirchoff COL stress is derived as shown below. Note that it is possible to define the fibril volume fraction distribution function and the pure COL strain energy density function can alternatively be defined by the Right Cauchy-Green deformation tensor (C), and the unit direction vector (n) using equation 3.40.

$$
\mathbf{S}^{\mathrm{COL}}=\int_{\theta=0}^{\pi} \int_{\varphi=0}^{2 \pi} \mathrm{R}(\theta, \Phi)(\mathbf{C}, \mathbf{n}) \mathrm{H}(\mathbf{C}, \mathbf{n}) \frac{\partial}{\partial \mathbf{C}} \Psi(\mathbf{C}, \mathbf{n}) \frac{1}{3} \sin \theta \mathrm{d} \theta \mathrm{d} \varphi
$$


The COL network elasticity tensor is similarly derived as follows.

$$
\mathbb{C}^{\mathrm{COL}}=\int_{\theta=0}^{\pi} \int_{\varphi=0}^{2 \pi} \mathrm{R}(\theta, \Phi)(\mathbf{C}, \mathbf{n}) \mathrm{H}(\mathbf{C}, \mathbf{n}) \frac{\partial^{2}}{\partial \mathbf{C}^{2}} \Psi(\mathbf{C}, \mathbf{n}) \frac{1}{3} \sin \theta \mathrm{d} \theta \mathrm{d} \varphi
$$

Immature bovine AC has been shown experimentally to exhibit an approximately linear stress-strain curve in tension. (Asanbaeva et al. 2008; Charlebois et al. 2004). Therefore, the following quadratic pure COL strain energy density function is proposed:

$$
\Psi=\Psi\left(E_{n}\right)=\frac{1}{2} E^{f}\left(E_{n}\right)^{2}
$$

where $\mathbf{E}^{f}$ is the true COL elastic modulus, and $\mathbf{E}_{\mathbf{n}}$ is the Lagrangian strain tensor for fibers in direction $\mathbf{n}$ as defined in equation 3.40. Proof of polyconvexity for this strain energy density function is found in Appendix A.1.3. With this selected pure COL strain energy density function COL fibril strain energy density function becomes

$$
W^{\mathrm{COL}}=\int_{\theta=0}^{\pi} \int_{\varphi=0}^{2 \pi} \frac{1}{6} \mathrm{R}(\theta, \Phi) \mathrm{H}\left(\mathbf{E}_{\mathbf{n}}\right) \mathbf{E}^{f}\left(\mathbf{E}_{\mathbf{n}}\right)^{2} \sin \theta \mathrm{d} \theta \mathrm{d} \varphi
$$

The COL fibril strain energy density function is derived from equation 3.45 giving the result shown in equation 3.46 . 


$$
\begin{gathered}
\mathbf{S}^{\mathrm{COL}=} \int_{\theta=0}^{\pi} \int_{\varphi=0}^{2 \pi} \mathrm{R}(\theta, \Phi)\left(\mathbf{E}_{\mathbf{n}}\right) \mathrm{H}\left(\mathbf{E}_{\mathbf{n}}\right)\left[\frac { \mathrm { E } _ { \mathrm { f } } } { 2 } [ \mathbf { n } \cdot [ [ ( ( \mathbf { F } _ { 0 } ^ { \mathrm { COL } } ) ^ { \mathrm { T } } \mathbf { C } \mathbf { F } _ { 0 } ^ { \mathrm { COL } } - \mathbf { I } ) ] \mathbf { n } ] ] \left[\mathbf{F}_{0}^{\mathrm{COL}}\right.\right. \\
\left.\left.\otimes \mathbf{F}_{0}^{\mathrm{COL}}\right]:[\mathbf{n} \otimes \mathbf{n}]\right] \frac{1}{3} \sin \theta \mathrm{d} \theta \mathrm{d} \varphi
\end{gathered}
$$

Where (:) is the double dot product and $\otimes$ is the dyadic product. Using equation 3.16 the second Piola-Kirchoff stress is transformed to Cauchy stress and implemented in FE analysis and solutions. Similarly, the COL network elasticity tensor is derived as follows.

$$
\begin{array}{r}
\mathbb{C}^{\mathrm{COL}}=\int_{\theta=0}^{\pi} \int_{\varphi=0}^{2 \pi} \mathrm{R}(\theta, \Phi) \mathrm{H}\left(\mathrm{E}_{\mathbf{n}}\right) \mathrm{E}_{\mathrm{f}}\left[\left[[\mathbf{n} \otimes \mathbf{n}] ;\left[\left(\mathrm{F}_{0}^{\mathrm{COL}}\right)^{\mathrm{T}} \otimes\left(\mathbf{F}_{0}^{\mathrm{COL}}\right)^{\mathrm{T}}\right]\right]\right. \\
\left.\otimes\left[[\mathbf{n} \otimes \mathbf{n}]:\left[\left(\mathrm{F}_{0}^{\mathrm{COL}}\right)^{\mathrm{T}} \otimes\left(\mathrm{F}_{0}^{\mathrm{COL}}\right)^{\mathrm{T}}\right]\right]\right] \frac{1}{3} \sin \theta \mathrm{d} \theta \mathrm{d} \varphi
\end{array}
$$

Using equation 3.30 the material elasticity tensor, $\left(\mathbb{C}^{\mathrm{COL}}\right)$ is implemented for $\mathrm{FE}$ analysis as the $\mathrm{COL}$ constituent material Jacobian matrix $\left(\mathbb{C}^{\mathrm{COL} J \mathrm{AC}}\right.$ ). 


\subsubsection{Ground Substance Matrix Material Model}

The MAT material is assumed to be stress free in the initial undeformed MAT constituent reference configuration. Mechanical material properties are governed by the right Cauchy Green deformation tensor $\mathbf{C}$, and a single material constant, the MAT shear modulus $\mu$ which has units of MPa. The following strain energy function is proposed for MAT:

$$
W^{\mathrm{MAT}}=\frac{1}{2} \mu\left[\left(\mathrm{I}_{1}(\mathbf{C})-3\right)-\ln \left(\mathrm{I}_{3}(\mathrm{C})\right)\right]
$$

where $\mathrm{I}_{1}(\mathrm{C})=\operatorname{tr}(\mathrm{C})$ is the first invariant and trace of the right Cauchy-Green deformation tensor and $\mathrm{I}_{3}(\mathbf{C})=\operatorname{det}(\mathbf{C})$ is the third invariant and determinant of right Cauchy-Green deformation tensor. Note that in the initial undeformed stress free reference configuration the MAT strain energy is zero. Proof of polyconvexity for the MAT strain energy function can be found in Appendix A.1.3. Using this strain energy function, and the derivation from Appendix A.1.1 the second PiolaKirchoff MAT stress is shown below.

$$
\mathbf{S}^{\mathrm{MAT}}=\mu\left(\mathbf{I}-\mathbf{C}^{-\mathrm{T}}\right)=\mu\left(\mathbf{I}-\mathbf{C}^{-1}\right)
$$

which is transformed to Cauchy stress as required for Abaqus FEA solutions and presented in indicial notation below.

$$
\mathrm{T}_{\mathrm{ij}}^{\mathrm{MAT}}=\frac{1}{\mathrm{~J}} \mathrm{~F}_{\mathrm{iA}}\left[\mu\left(\delta_{\mathrm{AB}}-\mathrm{C}_{\mathrm{AB}}^{-1}\right)\right] \mathrm{F}_{\mathrm{jB}}
$$


Using the derivation from Appendix A.1.2 the MAT material elasticity matrix is derived as shown below.

$$
\mathbb{C}_{\mathrm{ABCD}}^{\mathrm{MAT}}=\mu\left[\mathrm{C}_{\mathrm{AC}}^{-1} \mathrm{C}_{\mathrm{BD}}^{-1}+\mathrm{C}_{\mathrm{AD}}^{-1} \mathrm{C}_{\mathrm{BC}}^{-1}\right]
$$

Or alternatively, in direct notation

$$
\mathbb{\mathbb { C }}^{\mathrm{MAT}}=\mu\left[\left[\mathbf{C}^{-1} \otimes \mathbf{C}^{-1}+\mathbf{C}^{-\mathrm{T}} \otimes \mathbf{C}^{-\mathrm{T}}\right]\right]
$$

Using equation 3.30 the MAT material elasticity tensor, $\left(\mathbb{C}^{\mathrm{MAT}}\right)$ is implemented for FE analysis as the MAT constituent material Jacobian matrix $\left(\mathbb{C}^{\text {MAT JAC }}\right)$. 


\section{Chapter 4: Proteoglycan Cauchy Stress Function and FE Implementation}

\subsection{Goals}

The objective of this work is to develop an appropriate Cauchy stress function for the Proteoglycan (PG) constituent of articular cartilage (AC), and thereafter to derive the associated material Jacobian matrix and implement the model in a finite element analysis (FEA) solver. A PG is a complex macromolecule composed of multiple chains of glycosaminoglycan (GAG) molecules that exist within the extracellular matrix (ECM) of AC. Mechanically, PGs create a swelling pressure due to both the repulsive electromechanical forces created by charge carrying GAG molecules and osmotic pressure created by a charge differential between GAG molecules and the surrounding medium. In AC, PG swelling stress is restrained by the collagen network and helps to resist compressive forces in vivo. Studies have shown that the immature and mature AC equilibrium aggregate modulus $\left(\mathrm{H}_{\mathrm{A}}\right)$ and $\mathrm{AC}$ compressive modulus $(\mathrm{E})$ may initially decrease then increase with increasing compressive strains (Ficklin et al. 2001; Wang et al. 2003; Williams et al. 2010). Therefore, for this study it is hypothesized that for large compressive strains (>15\% - 45\%) the COL network should no longer be in tension in the direction of the loading axis after reaching a sufficient compressive strain (>15\% - 45\%). 


\subsection{Experimental Data Source}

Theoretical calculated data points for the Poisson-Boltzmann-cell (PBcell) model predictions (Buschmann and Grodzinsky 1995) were compared with experimentally measured isolated swamp rat chondrosarcoma PG swelling pressure data (Williams and Comper 1990). Experimental data for newborn, native (D0) bovine calf $A C$ compressive and biochemical properties were taken from my previous study (Williams et al. 2010) with the details briefly summarized here.

Full thickness bovine newborn (1-3 weeks) calf AC blocks were harvested from the medial and lateral ridges of the patellofemoral groove (PFG). Superficial $(\mathrm{S})$ and middle $(\mathrm{M})$ layers were sliced $(\sim 0.5 \mathrm{~mm}$ thick) from the articular surface using a vibratome (Figure 4.1). The slices were punched into discs for sequential confined compression (CC) (4.8mm disc) and unconfined compression (UCC) testing (3.2 $\mathrm{mm}$ disc). Samples were submerged in phosphate buffered saline with protease inhibitors (PBS+PI) and tested according to established protocols (Chen et al.2001; Williamson et al. 2001; Ficklin el al. 2007). 

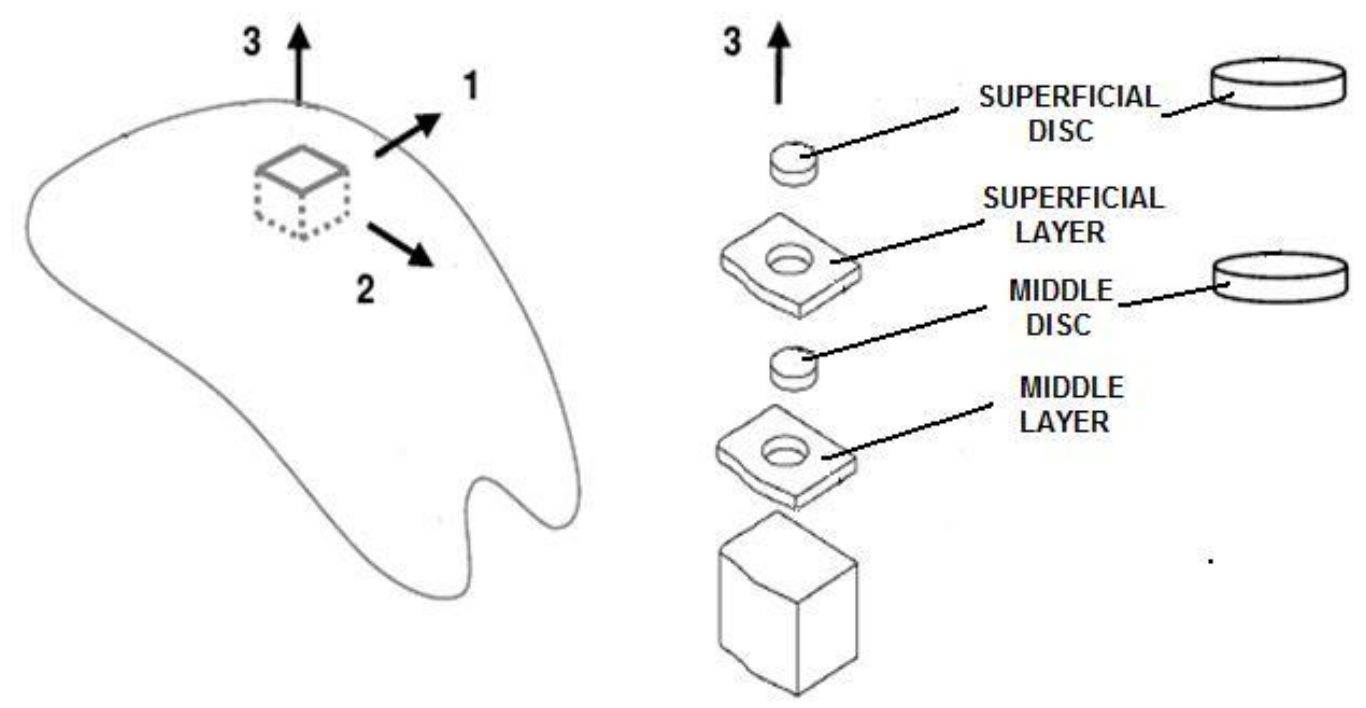

Figure 4.1. Compression and biochemical specimen preparation schematic showing a harvested full-thickness explant block. Slices were taken from the superficial and middle layers, and one disc was punched from each slice. Directions 1, 2, and 3 represent three mutually orthogonal directions.

Following compression testing, specimens were tested for biochemical properties which were normalized to initial sample wet weight $\left(\mathrm{WW}_{\mathrm{i}}\right)$. Cell and COL contents were calculated using ratios of 7.7 pgDNA/cell (Kim et al.1988) and $7.25 \mathrm{~g} \mathrm{COL} / \mathrm{g}$ hydroxyproline (Herbage et al. 1977; Pal et al. 1981), respectively. Average D0 constituent mass $/ W W_{i}$ results are shown below in Table 4.1.

Table 4.1. Biochemistry analysis results showing constituent (H2O, GAG, and $\mathrm{COL}$ ) masses normalized to initial wet weight for D0 superficial and middle layers. (Williams et al. 2010)

\begin{tabular}{|c|c|c|}
\hline Constituent & D0 Superficial Layer & D0 Middle Layer \\
\hline $\mathrm{H} 2 \mathrm{O}(\%$ tissue mass $)$ & 89.34 & 86.63 \\
\hline GAG $\left(\% \mathrm{WW}_{\mathrm{i}}\right)$ & 3.28 & 4.76 \\
\hline COL $\left(\% \mathrm{WW}_{\mathrm{i}}\right)$ & 5.53 & 7.15 \\
\hline
\end{tabular}




\subsection{Methods}

\subsubsection{Proteoglycan Cauchy Stress Function}

To develop a polyconvex FEA implementable continuum level PG Cauchy stress function, first an appropriate theoretical PG swelling stress model was selected. The PB-cell model was chosen as an accurate PG swelling stress model. In order to clearly express stresses the cylindrical coordinate system in Figure 4.1 is used for the remainder of this section.

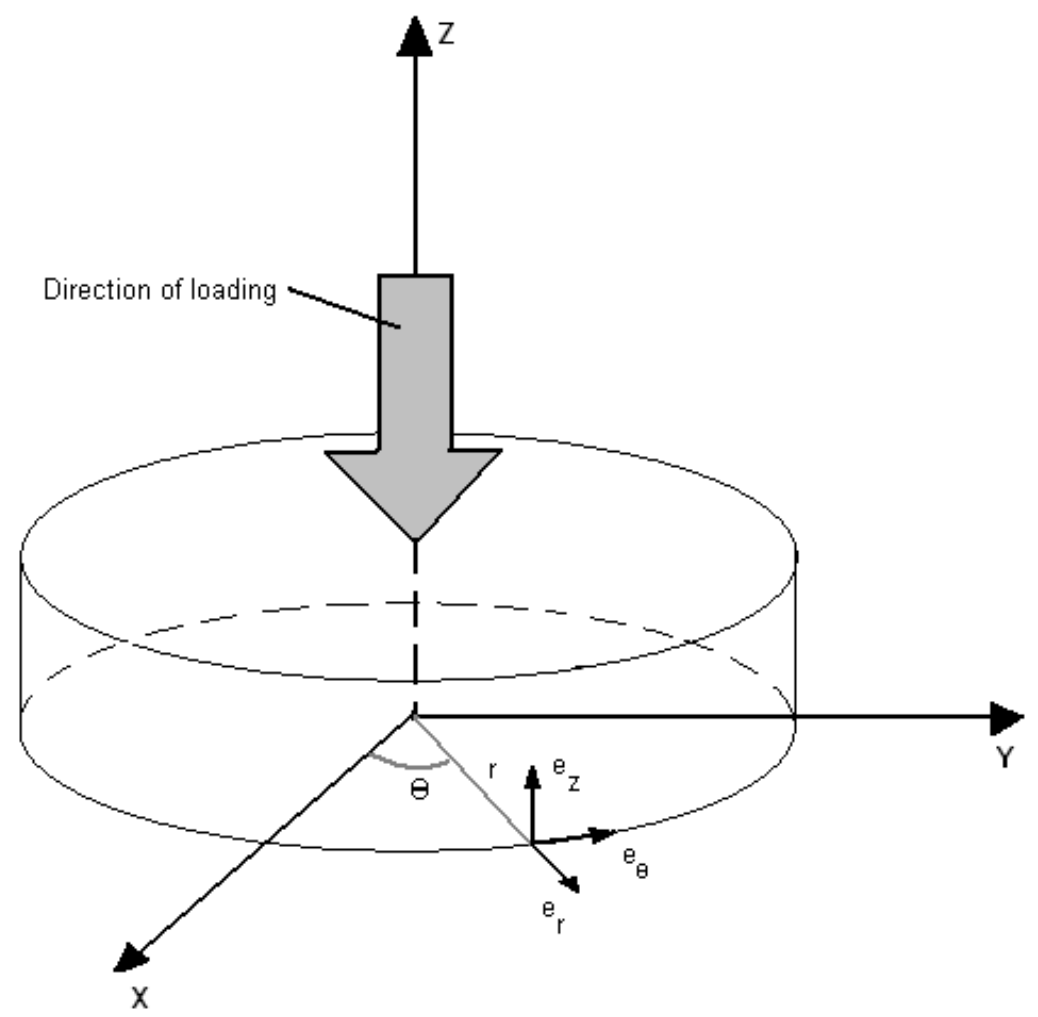

Figure 4.2 Cartilage specimen showing rectangular and cylindrical coordinate systems. For this study loading is always in the z-direction. 
To evaluate the effectiveness of different GAG Cauchy stress models, a constitutive AC model was developed according to the stress balance hypothesis shown in equation 4.1 .

$$
\mathbf{T}^{\mathrm{SM}}=\mathbf{T}^{\mathrm{PG}}+\mathbf{T}^{\mathrm{COL}+\mathrm{MAT}}
$$

where $\mathbf{T}^{\mathrm{SM}}$ is the experimentally measured Cauchy solid matrix stress in CC or UCC calculated using aggregate modulus $\left(\mathrm{H}_{\mathrm{A}}\right)$, or Young's modulus $(\mathrm{E})$, respectively. $\mathbf{T}^{\mathrm{PG}}$ is the predicted PG Cauchy stress, and $\mathbf{T}^{\mathrm{COL}+\mathrm{MAT}}$ is the combined predicted COL and MAT stress. Note that for this model, since it is only necessary to evaluate the PG Cauchy stress function, and not the individual COL and MAT constituent predictions, COL and MAT predictions are grouped together. It is hypothesized that COL fibers cannot support a compressive stress. Therefore, if a negative COL+MAT stress is predicted it is assumed that the compressive stress is carried solely by the MAT constituent.

The constitutive model was used to reproduce experimental conditions for CC and UCC loading conditions. For the UCC loading condition, the lateral boundary was assumed to be in equilibrium (i.e. not moving) and traction free. Therefore a lateral COL+MAT stress must be present to counteract the PG swelling stress present at the lateral boundary. UCC stress normal to the radial surface was calculated according to the following relationship

$$
\mathbf{T}_{\mathrm{rr}}^{\mathrm{PG}}=-\mathbf{T}_{\mathrm{rr}}^{\mathrm{COL}+\mathrm{MAT}}
$$

where $\mathbf{T}_{\mathrm{rr}}^{P G}$ is the PG Cauchy stress at the lateral boundary in the $r$ direction, and $\mathrm{T}_{\mathrm{rr}}^{\mathrm{COL}+\mathrm{MAT}}$ is the COL+MAT Cauchy stress required to satisfy the prescribed 
boundary conditions in the $r$ direction. In the direction of loading, applied stress $\left(\mathbf{T}_{\mathrm{zz}}^{\mathrm{App}}\right)$, is calculated as the sum of constituent stress as follows

$$
\mathrm{T}_{\mathrm{zz}}^{\mathrm{App}}=\mathrm{T}_{\mathrm{zz}}^{\mathrm{PG}}+\mathbf{T}_{\mathrm{zz}}^{\mathrm{COL}+\mathrm{MAT}}
$$

Note that $\mathbf{T}_{z z}^{P G}$ is predicted using a PG swelling stress model, so $\mathbf{T}_{z z}^{\mathrm{COL}+\mathrm{MAT}}$ is the only unknown quantity in equation 4.3. The COL+MAT stress responses were calculated for individual experimental explants and then averaged for strain level and layer. A description of the methods used to determine the determinant of the deformation gradient for each sample is provided in Appendix A.1.5.

Swelling stress data points from (Buschmann and Grodzinsky1995) were estimated for a GAG molecule radius of $0.55 \mathrm{~nm}$ proposed by Ogsten et al. (1973) and GAG intercharge distance of $0.51 \mathrm{~nm}$ proposed by GAG structural models (Comper and Laurent 1978; Preston et al. 1972). A continuous polynomial curve was fit determined using a least squares algorithm in Excel (Microsoft v. 2007). Alternative GAG interchange distance and GAG molecule radius values were also considered as inputs to the PB-cell model. However, for other GAG parameters the corresponding COL+ MAT constituent stress predictions stayed in tension for compressive strains up to $45 \%$. Since this validated the hypothesis that COL+MAT constituent stress should go into compression for compressive strains (> 15\% - 45\%) the alternative PB-cell parameter models were rejected. Curve fits to the theoretical PB-cell predictions for PG osmotic swelling pressure were developed using the form shown in equation 4.4:

$$
\pi^{\mathrm{PG}}=\alpha_{1}\left(\rho^{\mathrm{PG}}\right)^{\alpha_{\mathrm{z}}}
$$


where $\pi^{\mathrm{PG}}$ is $\mathrm{PG}$ osmotic swelling pressure $(\mathrm{KPa}), \rho^{\mathrm{PG}}$ is the current configuration PG density $(\mathrm{mg} / \mathrm{ml})$ and $\alpha_{1}$ and $\alpha_{2}$ are material constants varied to obtain the best overall curve fit and osmotic swelling stress prediction.

Potential PG swelling stress curve fits were evaluated based on the correlation coefficient $\left(\mathrm{R}^{2}\right)$ with PB-cell theoretical predictions. The predicted compressive strain level of the COL and MAT tension to compression transition was also considered, with the objective of predicting a transition from tension to compression for the COL+MAT constituent under large compressive strains.

A Cauchy stress function was selected based on the selected PG osmotic pressure curve fit according to the following relationship.

$$
\pi^{\mathrm{PG}}=-\mathbf{T}^{\mathrm{PG}}
$$

Therefore PG Cauchy stress using the curve fit in equation 4.4 is

$$
\mathbf{T}^{\mathrm{PG}}=-\alpha_{1}\left(\rho^{\mathrm{PG}}\right)^{\alpha_{\mathrm{Z}}} \mathbf{I}
$$

where $\rho^{\mathrm{PG}}$ is the current configuration GAG apparent density, and $\mathbf{I}$ is the identity tensor. Because PG density changes with compressive strain, it is desirable to implement a PG model which uses the experimentally measured reference configuration PG density. Applying the continuity relationship from equation 3.8 yields

$$
\mathbf{T}^{\mathrm{PG}}=-\alpha_{1}\left(\frac{\rho_{0}^{\mathrm{PG}}}{\mathrm{I}}\right)^{\mu_{\mathrm{Z}}} \mathbf{I}
$$

where $\rho_{0}^{\mathrm{GAG}}$ is the experimentally measured reference configuration PG apparent density. Note that because apparent density is used, there is no need to multiply 
Cauchy stress by a fluid volume fraction. The PG strain energy density function can be determined by integrating the Cauchy stress function with respect to the Jacobian using the proof in Appendix A.1.4. Therefore, the PG strain energy density function is:

$$
W^{P G}=\alpha_{2} \alpha_{1} \frac{\left(\rho_{0}^{P G}\right)^{u_{n}}}{\mathrm{~J}^{\left(\alpha_{z}-1\right)}}
$$

Derivations of terms necessary for the FE implementation of the PG constituent can be found in Appendix A. Derivation of the Cauchy stress from the PG strain energy function is shown in Appendix A.4.1. Derivation of the PG material elasticity tensor is shown in Appendix A.4.2. Derivation of the PG material Jacobian matrix is shown in Appendix A.4.3.

\section{Biochemical Composition}

To examine the effects of biochemical composition on constituent stress predictions the COL/GAG mass ratio was calculated for each experimental specimen as shown below.

$$
\frac{\mathrm{COL}}{\mathrm{GAG}}=\frac{\operatorname{mass} \mathrm{COL}(\mathrm{g} \mathrm{WWW})}{\text { mass GAG }(96 \mathrm{WWW})}
$$

where $\%$ WW refers to the constituent mass divided by the total tissue mass. The calculated COL/GAG mass ratio was correlated against COL+MAT predicted stress at 30\% CC and UCC strains. For each case linear regression with t-test analysis of the regression slope was performed. 


\section{Polyconvexity}

The PG strain energy function was tested for polyconvexity to help ensure numerical stability in FE analysis. The derivation and resulting constraints on $\alpha_{1}$ and $\alpha_{2}$ are shown in Appendix A.4.4.

\subsection{Results}

\subsection{Proteoglycan Swelling Stress Function Curve Fits}

The PG swelling stress function in equation 4.6 was evaluated for several different values of $\alpha_{1}$ and $\alpha_{2}$. The associated correlation coefficients $\left(R^{2}\right)$, material constants, and rejection/acceptance determinations are recorded in Table 4.2. Additionally, a graphical comparison of the proposed curve fits is shown in Figure 4.3.

Table 4.2. Results of curve fits to PB-cell model predictions for a GAG molecule radius of $0.55 \mathrm{~nm}$ and GAG interchange distance of $0.51 \mathrm{~nm}$.

\begin{tabular}{|c|c|c|c|}
\hline$\alpha_{1 \cdot}\left(\frac{\mathrm{N}^{2} \mathrm{~m}^{2.5}}{\mathrm{~m}^{2} \cdot \mathrm{mg}^{2.5}}\right)$ & $\alpha_{2}$ & $\mathrm{R}^{2}$ & Accepted/Rejected \\
\hline 22.2 & 2.00 & 0.92 & Rejected, $\mathrm{R}^{2}$ too low \\
\hline 8.80 & 2.25 & 0.95 & Rejected, $\mathrm{R}^{2}$ too low \\
\hline 0.400 & 3.00 & 0.99 & $\begin{array}{c}\text { Rejected, Over prediction of PG swelling stress for high } \\
\text { PG concentrations }\end{array}$ \\
\hline 2.87 & 2.50 & 0.98 & Accepted \\
\hline
\end{tabular}


Note that for the PB cell model prediction corresponding to $\alpha_{1}=0.400$ and $\alpha_{2}=3.00$ was rejected because predicted GAG stresses were too high relative to the applied stresses causing the COL+MAT tension to compression transition to not occur for either CC or UCC strains up to $45 \%$.



Figure 4.3. Comparison of curve fits to PB-cell model predictions for a GAG molecule radius of $0.55 \mathrm{~nm}$ and GAG interchange distance of $0.51 \mathrm{~nm}$. The curve fit parameters $\alpha_{1}$ and $\alpha_{2}$ correspond to equation 4.6.

Based on the decided curve fit the PG swelling stress model becomes

$$
\pi^{\mathrm{PG}}=2.87\left(\rho^{\mathrm{PG}}\right)^{2.5}
$$

And consequently continuum level PG Cauchy stress becomes

$$
\mathbf{T}^{\mathrm{PG}}=-2.87\left(\frac{\rho_{0}^{\mathrm{PG}}}{\mathrm{J}}\right)^{2 . \mathrm{s}} \mathbf{I}
$$




\subsubsection{Constituent Stress Predictions in Compression}

PG, COL+MAT and SM stress responses were calculated at equilibrium 0\%, 15\%,30\%, and $45 \%$ CC and UCC strains using the PG Cauchy stress shown in equation 4.11 , the stress balance hypothesis shown in equation 4.1 , and experimental results (Williams et al. 2010). The constituent stress results are shown for CC of the S and M layers (Figure 4.4) and for UCC of the S and M layers (Figure 4.5).

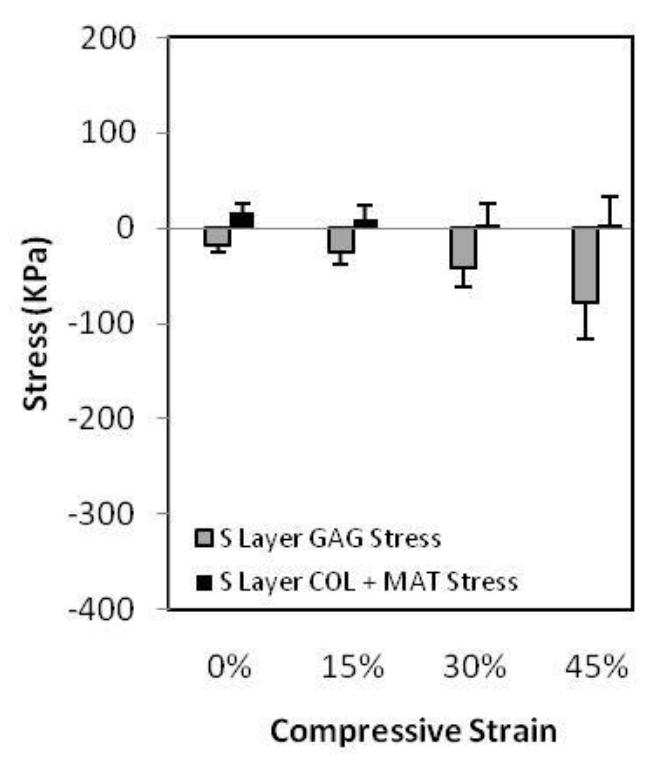

(A)

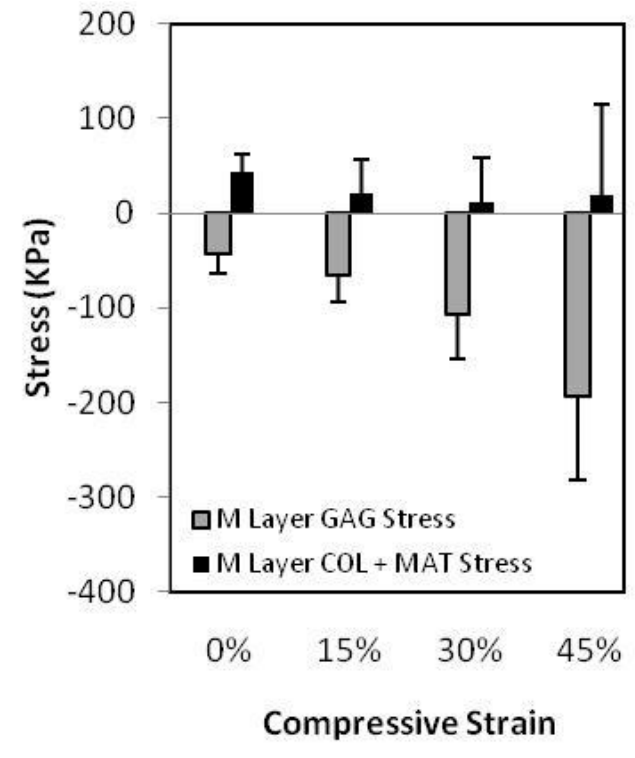

(B)

Figure 4.4. Constituent stress results for D0, (A) superficial layer, and (B) D0 middle Layer, samples loaded in confined compression at increasing levels of compressive strain. Note the difference in stress magnitude between superficial and middle layers. Mean \pm SD: $n=10,23$. 


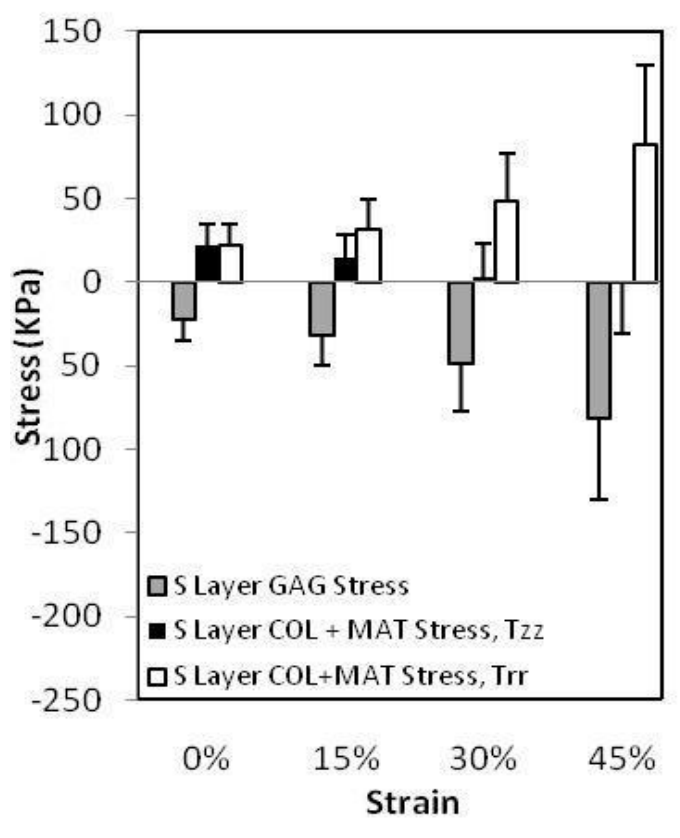

(A)

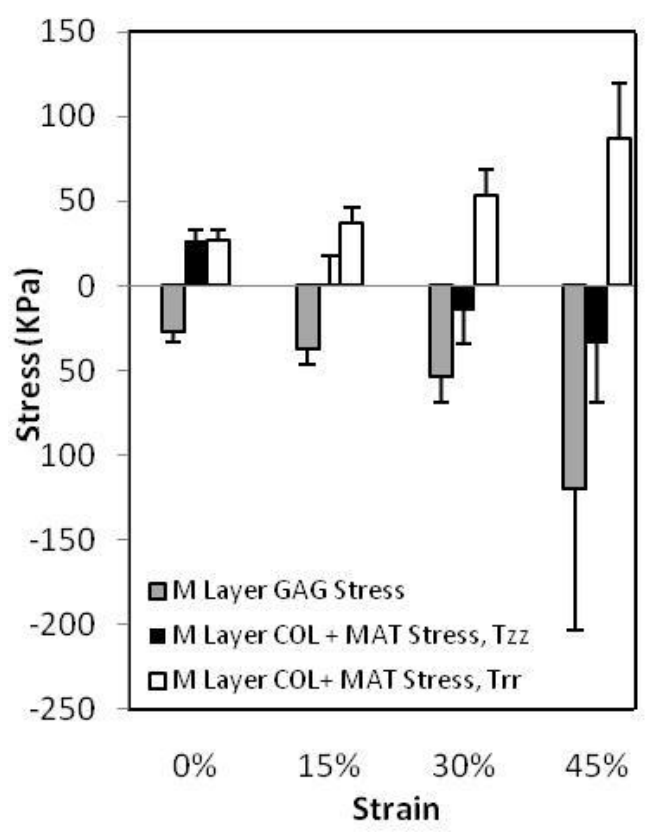

(B)

Figure 4.5. Constituent stress results for (A) D0 superficial layer and (B) D0 middle Layer samples from the selected PG Cauchy stress model in unconfined compression at increasing levels of compressive strain. Mean \pm SD: $n=11,6$.

The COL/GAG mass ratio of individual explants was shown to affect the explant specific constituent stress predictions. Explants with higher COL/GAG mass ratios generally predicted lower COL+MAT stress predictions in compressive loading compared to explants with lower COL/GAG mass ratios. The correlations between COL/GAG mass ratio and predicted COL+MAT stress are shown for CC explants (Figure 4.6) and UCC explants (Figure 4.7). 


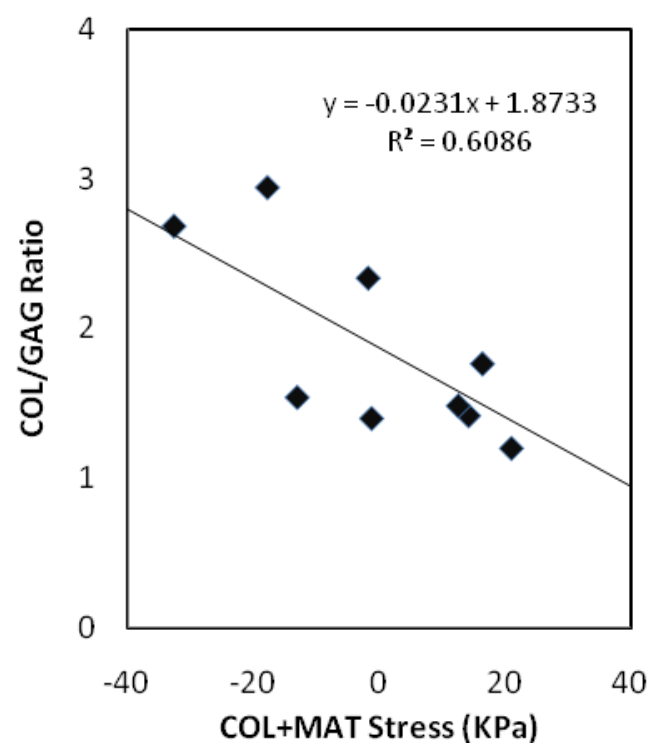

(A)



(B)

Figure 4.6. Correlation between COL/GAG mass ratio and predicted $\mathrm{COL}+$ MAT constituent stress for (A) DO S Layer, and (B) DO M Layer at 30\% CC strain. T-test of the linear regression slope gives $p=0.0893$ and $p=0.0623$ for $S$ and $M$ layers, respectively. Linear regression fit and $\mathrm{R}^{2}$ are shown

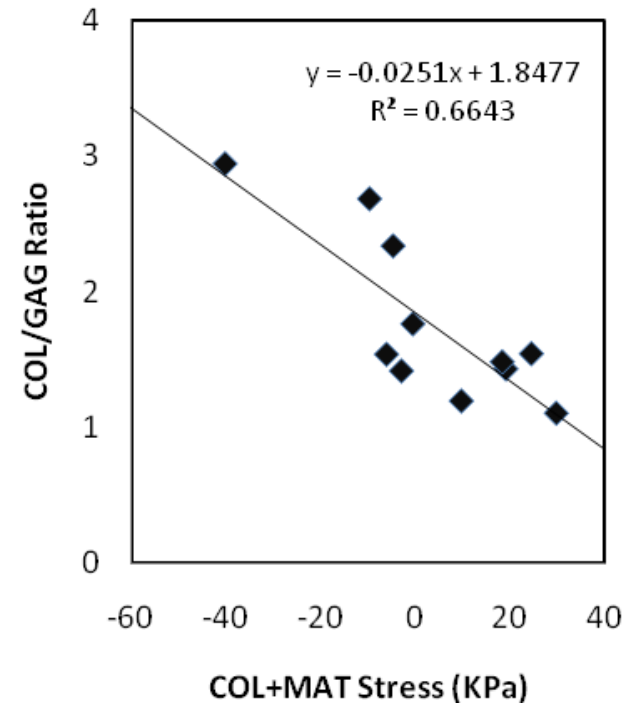

(A)

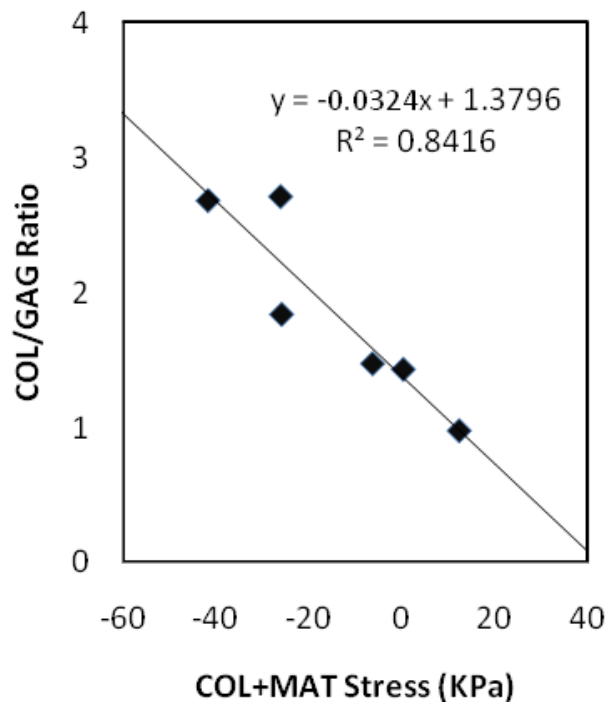

(B)

Figure 4.7. Correlation between $\mathrm{COL} / \mathrm{GAG}$ mass ratio and predicted $\mathrm{COL}+$ MAT constituent stress for (A) DO S Layer, and (B) D0 M Layer at 30\% UCC strain. T-test of the linear regression slope gives $p=0.0007$ and $p=0.0018$ for $S$ and $M$ layers, respectively. Linear regression fit and $R^{2}$ are shown 


\subsection{Discussion}

\subsubsection{PG Swelling Stress Function Curve Fits}

Overestimation of PG swelling stress, particularly at low FCD or PG concentration creates a problem for constitutive AC models. For some models COL fibers are predicted to remain in tension upwards of $45 \%$ UCC strain (Oungoulian 2007). The PB-cell model presents a possible solution as a physically relevant model of PG osmotic swelling pressure that has been shown to predict lower PG swelling stresses compared to other models (Buschmann and Grodzinsky 1995). Additional studies also suggest that the PB-cell model results are accurate. For example, a molecular GAG model (Bathe et al. 2005) for 16 or 32 disaccharide unit chains of chondroitin sulfate (typically 20-60 for cartilage aggrecan) provides a close agreement to the selected PB-cell model PG swelling stress predictions. The selected PB-Cell model is also in agreement with the experimental PG osmotic swelling pressure results and PB model predictions of Ehrlich et al. (1998).

Results indicate that the curve fit for equation 4.4 to the PB cell model

with values for $\alpha_{1}$ and $\alpha_{2}$ of $2.87 \frac{\mathrm{N} \cdot \mathrm{ml}^{2.5}}{\mathrm{~m}^{2} \cdot \mathrm{mg}^{2.5}}$ and 2.5 , respectively, provides the best PG swelling stress prediction and consequently more reasonable constituent stress predictions for D0 bovine newborn calf $A C$. This conclusion is based on the hypothesis that the COL + MAT constituent should transition from tensile stress to compressive stress for compressive strains (>15\% - 45\%). 
Different values of $\alpha_{1}$ and $\alpha_{2}$ were also considered, but were rejected either due to high PG swelling stress predictions, or low correlation coefficients to experimental PG osmotic swelling pressure data. Various PB-cell parameters were also considered, but a GAG molecule radius of $0.55 \mathrm{~nm}$ and GAG interchange distance of $0.51 \mathrm{~nm}$ resulted in the best constituent stress predictions for native newborn bovine AC. The Cauchy stress equation developed based on these parameters provides a FEA implementable, polyconvex strain energy function for the PG constituent of AC.

\subsubsection{Constituent Stress Predictions in Confined and Unconfined Compression}

The selected PG swelling stress model predicts a tension to compression transition of COL+MAT constituent stress in the direction of loading (z-direction) in UCC. In the radial direction in UCC, COL+MAT stresses are initially tensile and increase with increasing UCC strain. In CC the COL+MAT stress predictions remain in tension for all experimental strain levels ( $0 \%, 15 \%, 30 \%, 45 \%)$. Therefore, it can be concluded that loading condition (CC vs. UCC) does have an effect on the tension to compression transition of $\mathrm{COL}+\mathrm{MAT}$ constituent stress in compression.

Biochemical composition of individual explants, and in particular the GAG/COL, mass ratio has an effect on the constituent stress responses. For $\mathrm{UCC}$, in the $\mathrm{S}$ and $\mathrm{M}$ layers, there was a negative correlation between $\mathrm{GAG} / \mathrm{COL}$ 
mass ratio and COL+MAT stress $(p<0.05)$. In CC, a similar trend was observed, yet significance was not found $(p>0.05)$. For the CC trend, additional data would likely show significance. Quantifying the GAG/COL mass ratio for a tissue group may provide an indication as to whether or not the COL+MAT constituent will transition from the tensile strain initially necessary for equilibrium to compressive strain during compressive loading. Samples with large amounts of GAG molecules and/or low COL content are less likely to have "slack" COL fibers when loaded in compression due to the increased PG swelling pressure. Also, samples with lower amounts of GAG molecules and/or high COL content are more likely to have "slack" COL fibers in compressive loading. This finding reinforces the profound effects of relative constituent concentrations on the biomechanical response of $\mathrm{AC}$. 


\section{Chapter 5: Material Parameter Determination}

\subsection{Goals}

The primary objective of this project is to develop a realistic continuum mechanics based FEA model of AC to model in vitro mechanical experiments for native explants and explants cultured in TGF- $\beta 1$ or IGF-1. Another objective of this project is to validate the resulting FEA model of $A C$ for multiple layers, culture treatments, and loading types. This overlapping validation protocol is intended to provide a more complete and comprehensive validation, compared to validating for a single experimental loading condition, group, or layer. A final objective of this project is to determine if there are changes in the true COL fiber modulus $\left(E_{F}\right)$ or ground matrix shear modulus $(\mu)$ with depth from the articular surface and for culture in growth factors IGF-1 and TGB- $\beta 1$. These goals seek to further develop the understanding of the constitutive mechanical effects of culture in IGF-1 and TGB- $\beta 1$ on AC tissue. 


\subsection{Experimental Data Source}

Experimental mechanical and biochemical data necessary for this study determination came from two different sources. Biochemical and mechanical data for D0 samples and samples cultured for 12 days in medium supplemented with growth factors (D12) then tested sequentially in compression (CC and UCC) is taken from (Williams et al. 2010) as described in Section 4.2. Mechanical data for D0 and D12 tensile specimens came from (Stender et al. 2011) with additional details for the tensile sample preparation outlined as follows.

Full thickness bovine newborn (1-3 weeks) calf AC blocks were harvested from the medial ridge, the lateral ridge, and the center of the patellofemoral groove (PFG) (Figure 5.1) S and M layers were sliced ( 0.5mm each) from the articular surface using a vibratome. Samples were cultured according to the methods of Asanbaeva et al. (2008).

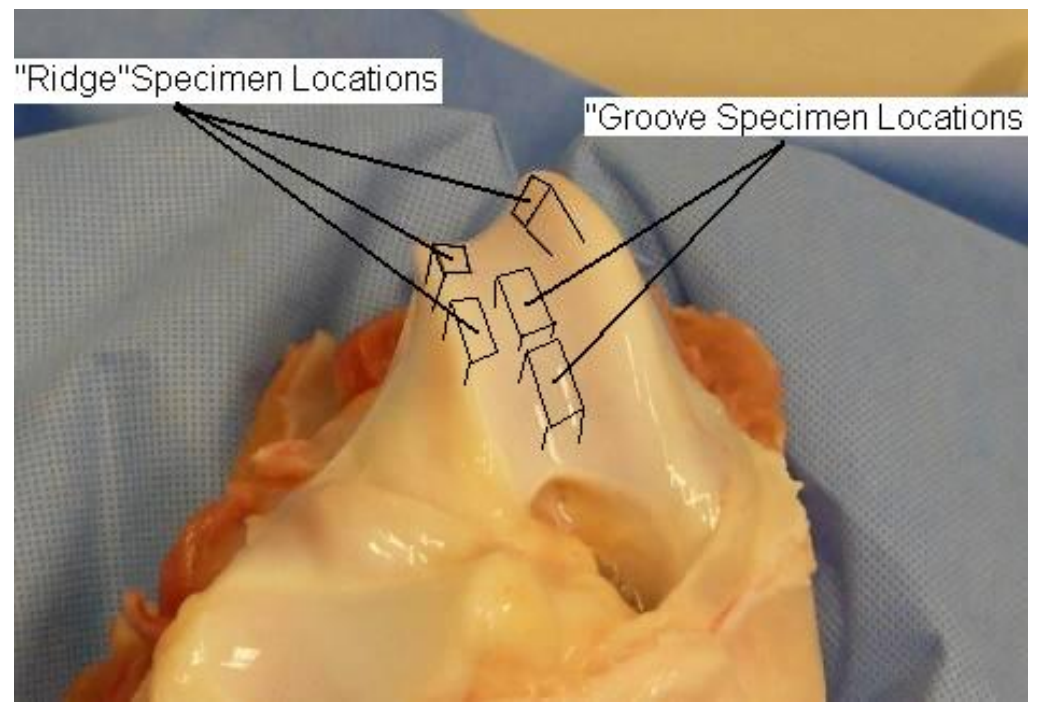

Figure 5.1. Patellofemoral groove showing different location for "groove" and "ridge" specimens. 
The slices were punched into a tapered "dog bone" strip with a gauge region of $4.0 \mathrm{~mm} \times 0.8 \mathrm{~mm}$ for tensile testing. For both ridge and groove harvest sites, the long axis of the sample was oriented in the anterior posterior direction. During mechanical testing, a tare strain was initially applied to each specimen, followed by a fixed displacement ( $5 \%$ then $10 \%$ tensile strain). Therefore, the total tensile strain magnitude is the tare strain plus the applied fixed displacement.

Analysis of variance (ANOVA) was used to determine the effect of strain level, $15 \%$ vs. $30 \%$ in UCC and $5 \%$ vs. $10 \%$ in tension and layer, S vs. M. Additionally, an ANOVA analysis was performed to determine the effect of direction on Poisson's rations in UCC ( $v_{31}$ vs. $v_{32}$ ) and strain level $15 \%$ vs. $30 \%$. Direction did not have a significant effect on Poisson's ratios in UCC ( $p>0.05)$. Therefore, Poisson's ratios $v_{31}$ and $v_{32}$ were averaged and a single averaged Poisson's ratio was assumed for each UCC group (D0, IGF-1, and TGF- $\beta 1$ ). Because AC exhibits a much higher Young's modulus (E), in tension compared to compression, (Soltz and Ateshian 2000; Williamson et al. 2003; Huang et al. 2005; Ficklin et al. 2007) $\mathrm{E}^{-}$is used to define Young's modulus in compression (UCC) and $\mathrm{E}^{+}$is used to define the Young's modulus in tension. For all D0 and D12 groups strain level (15\% vs. $30 \%$ in UCC and $5 \%$ vs. $10 \%$ in tension) was determined to not have a significant effect on UCC Poisson's ratio $(v)$, UCC modulus $\left(\mathrm{E}^{-}\right), \mathrm{CC}$ aggregate modulus $\left(\mathrm{H}_{\mathrm{A}}\right)$, or tensile modulus $\left(\mathrm{E}^{+}\right)(p>0.05)$. Therefore, CC, UCC, and tension mechanical and biochemical data was averaged across strain level. The model was fit to the average strains of the 
resulting data set with $22.5 \%$ strain in UCC and CC and $7.5 \%$ strain plus tare strain in tension.

Experimental data was assembled into six groups: D0 S and M layers, D12: IGF-1 S and M layers, and D12: TGF- $\beta 1$ S and M layers each with corresponding $\mathrm{E}^{-}, \mathrm{H}_{\mathrm{A}}$, and $v$ averaged to $22.5 \% \mathrm{UCC}$ strain and $\mathrm{E}^{+}$averaged to 7.5\% plus tare strain. Since no ridge location tensile data was available, D12 $\mathrm{E}^{+}$ values were scaled using either the S or M layer groove to ridge modulus ratios determined from Stender et al. (2011) Following compression testing, ridge specimens were tested for biochemical properties and normalized to initial sample wet weight $\left(\mathrm{WW}_{\mathrm{i}}\right)$. Cell and COL contents were calculated using ratios of 7.7 pgDNA/cell (Kim et al.1988) and $7.25 \mathrm{~g} \mathrm{COL/g} \mathrm{hydroxyproline} \mathrm{(Herbage} \mathrm{et} \mathrm{al.}$ 1977; Pal et al. 1981). Tensile specimens were assumed to have the same mean biochemical properties as the ridge compression specimens listed in Table 4.1.

\subsection{Methods}

AC was assumed to be composed of three constituents (GAG, MAT, and COL). A FEA model was developed using a UMAT to define material behavior in the FEA solver Abaqus (SIMULIA. Providence, RI). The FEA models developed for this study model initial and displacement controlled equilibrium experimental boundary conditions for CC, UCC and tension tests. 


\section{Finite Element Analysis}

Initially the constitutive FEA model is a sum of the individual constituent reference configurations. In the PG constituent reference configuration $\left(\kappa_{0}^{\mathrm{PG}}\right)$, there is a negative PG stress due to the initial GAG concentration. Both COL and MAT constituents have zero stress constituent reference configurations $\mathbf{k}_{0}^{\text {COL }}$ and $\boldsymbol{\kappa}_{0}^{\mathrm{MAT}}$, respectively (Figure 5.2).

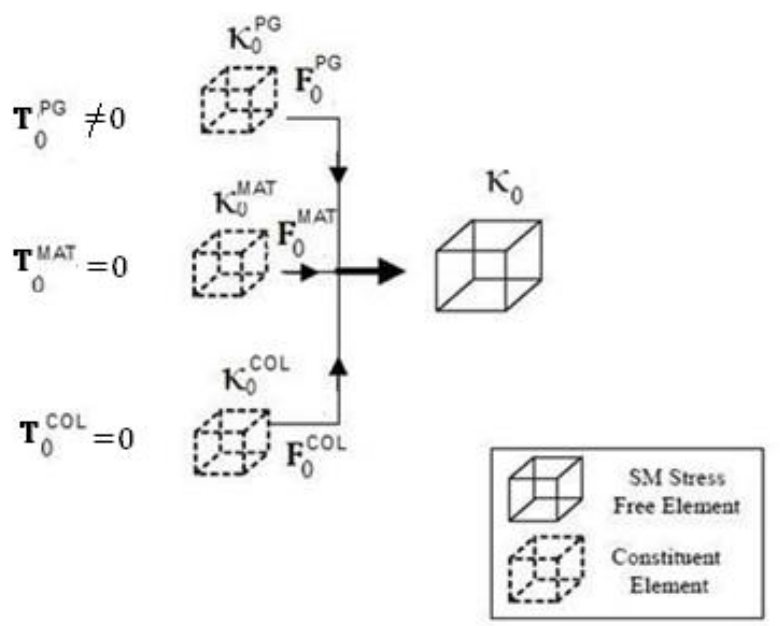

Figure 5.2. Initial constituent reference configurations and the corresponding initial stresses for PG, MAT and COL constituents.

Because of the nonzero PG constituent reference configuration stress, prior to all analyses an equilibrium step must be solved wherein each constituent undergoes an initial deformation, resulting in a stress free SM. Note that because all constituents are combined into the SM, and therefore subject to the immobility constraint, all constituents will undergo the same initial deformation. A zero SM stress configuration was solved for analytically in Abaqus by declaring a stress free initial condition and solving for the resulting constituent strains. 
Although the model can account for anisotropic fiber distributions, for this experimental data set, an isotropic fiber distribution was used for both $\mathrm{S}$ and $\mathrm{M}$ layers. The Benninghoff structure, which proposes an anisotropic COL fiber distribution particularly in the S layer, is well documented for mature AC across species (Rieppo et al. 2009; Zambrano et al. 1982; Van Turnhout et al. 2008). Other studies suggest that this structure is absent for early postnatal development, and therefore COL fiber distribution is isotropic in S an M layers across species (Van Turnhout et al. 2010; Rieppo et al. 2009; Julkunen et al. 2010). An experimental study tested $S$ layer newborn bovine calf AC in two orthogonal directions and did not find a significant difference in tensile modulus (Williamson et al. 2003). Therefore, an isotropic COL fiber distribution was assumed for this study.

To model experimental conditions, and to save computational time, single element C3D8 eight node, full integration, linear brick element models were created with appropriate boundary conditions for CC, UCC, and tension experiments. Increasing the number of elements had no effect on the FEA results (Appendix B.5.6). For UCC and Tension cases the boundary conditions are analogous to a "cube in a corner" (Figure 5.3) wherein one face in each of three mutually orthogonal directions was unconstrained and a displacement boundary condition was applied to one of the three free surfaces. Each of the constrained faces was defined to have a zero displacement in the direction normal to the given surface. For the CC condition, each of the four lateral surfaces was defined to have zero displacement in a direction normal to the respective face and a displacement was applied to the top surface. 


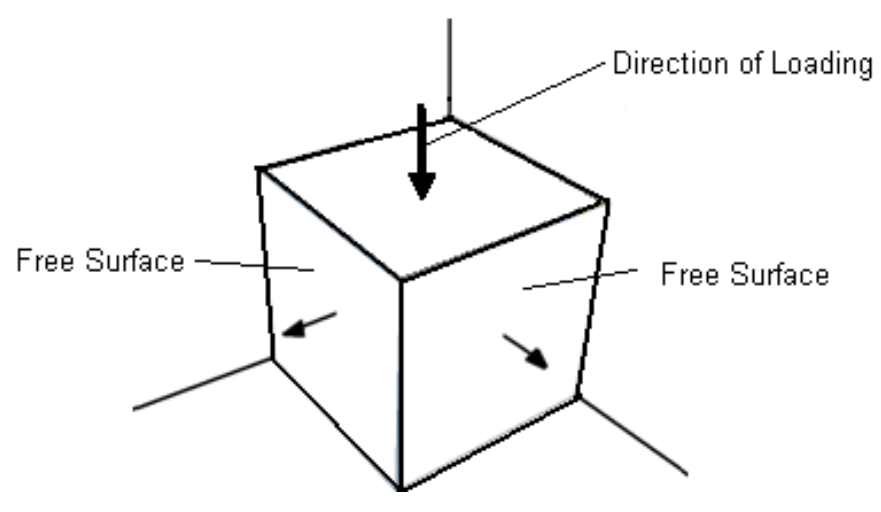

Figure 5.3. A Pictorial representation of UCC and Tensile "cube in a corner" boundary conditions.

Constituent specific material constants as well as experimentally measured biochemical parameters were required as inputs to the model for each group. A table of the required material constants, the associated units, and the method of determining each constant is shown in Table 5.1.

Table 5.1. Material constants necessary for the constitutive FE model of AC with the appropriate units, and method of determination.

\begin{tabular}{|c|c|c|}
\hline Material Constant & Units & Method of Determination \\
\hline $\mathrm{E}_{\mathrm{f}}$ COL fiber modulus & $\mathrm{MPa}$ & Fit to experimental data \\
\hline$\mu$, MAT shear modulus & $\mathrm{MPa}$ & Fit to experimental data \\
\hline$\Phi_{\mathrm{f}}$, COL fiber volume fraction & None & Experimentally measured \\
\hline$P_{0}^{P G}$, Reference Configuration $\mathrm{GAG}$ \\
density & $\frac{\mathrm{mg}}{\mathrm{ml}}$ & Experimentally measured \\
\hline$\alpha_{1}$ & $\frac{\mathrm{N} \cdot \mathrm{ml}^{2.5}}{\mathrm{~m}^{2} \cdot \mathrm{mg}^{2.5}}$ & Fit to PG swelling pressure data \\
\hline$\alpha_{2}$ & None & Fit to PG swelling pressure data \\
\hline
\end{tabular}

\section{Validation}

An optimization script was coded in Python (Python Software Foundation, v3.2. alpha1) to vary the true $\mathrm{COL}$ fiber modulus $\left(\mathrm{E}_{\mathrm{f}}\right)$ and MAT shear modulus $(\mu)$ 
in order to obtain the best fit to experimental mechanical data by minimizing an objective function as shown in equation 5.1

$$
\varphi=\left\{\begin{array}{lr}
\left(v_{\text {model }}-v_{\exp }\right)^{2}+\left(E^{-}{ }_{\text {model }}-E^{-}{ }_{\exp }\right)^{2} & \text { fit to UCC } \\
\left(E_{\text {model }}^{+}-E_{\exp }^{+}\right)^{2} & \text { fit to tensile }
\end{array}\right.
$$

where $\varphi$ is the value of the objective function and the subscripts model and exp represent predicted values and experimentally measured values, respectively. In order to develop a more comprehensive validation of the model, validation was carried out for each of the 6 experimental groups, and with each of the 3 following methods:

1. Fit to UCC - $E_{f}$ and $\mu$ were varied to obtain the best fit to experimentally determined UCC mechanical parameters $\mathrm{E}^{-}$and $v$. From the determined $\mathrm{E}_{\mathrm{f}}$ and $\mu$ values, $\mathrm{E}^{+}$and $\mathrm{H}_{\mathrm{A}}$ were predicted.

2. Fit to Tension - $E_{f}$ and $\mu$ were varied to obtain the best fit to experimentally determined tensile modulus $\mathrm{E}^{+}$. For the determined $\mathrm{E}_{\mathrm{f}}$ and $\mu$ values, $\mathrm{E}^{-}$and $\mathrm{H}_{\mathrm{A}}$ were predicted.

3. Simultaneous fit $-\mathrm{E}_{\mathrm{f}}$ and $\mu$ were varied to simultaneously match UCC parameters $\mathrm{E}^{-}$, and $v$ and tensile modulus $\mathrm{E}^{+}$such that percent errors were approximately the same for each parameter. $\mathrm{H}_{\mathrm{A}}$ was predicted.

In order to quantitatively asses the overall effectiveness of the different fit methods the complete objective function was shown in equation 5.2 was calculated for each group and fit method. 


$$
\varphi=\sqrt{\left(v_{\text {model }}-v_{\text {exp }}\right)^{2}+\left(E^{-}{ }_{\text {model }}-E^{-}{ }_{\exp }\right)^{2}+\left(E_{\text {model }}^{+}-E^{+}{ }_{\exp }\right)^{2}+\left(\mathrm{H}_{A_{\text {model }}}-\mathrm{H}_{A_{\text {Exp }}}\right)^{2}}
$$

To ensure physically reasonable parameter determination, upper and lower bounds were enforced on the values of $E_{f}$ and $\mu\left(0.001 \mathrm{MPa}<\mathrm{E}_{\mathrm{f}}<\right.$ $3000 \mathrm{MPa})$ and $(0.001 \mathrm{MPa}<\mu<1000 \mathrm{MPa})$.

\subsection{Results}

\subsubsection{Material Parameters}

Material parameters were determined according to the methods outlined in section 5.3. The results for the $S$ and $M$ layers are shown below in Figure 5.3 and. For all groups and fitting methods MAT shear modulus $\mu$, was determined to be $0.001 \mathrm{MPa}$. Numerical values for the determined $\mathrm{E}_{\mathrm{f}}$ and $\mu$ can be found in Appendix B.5.5 


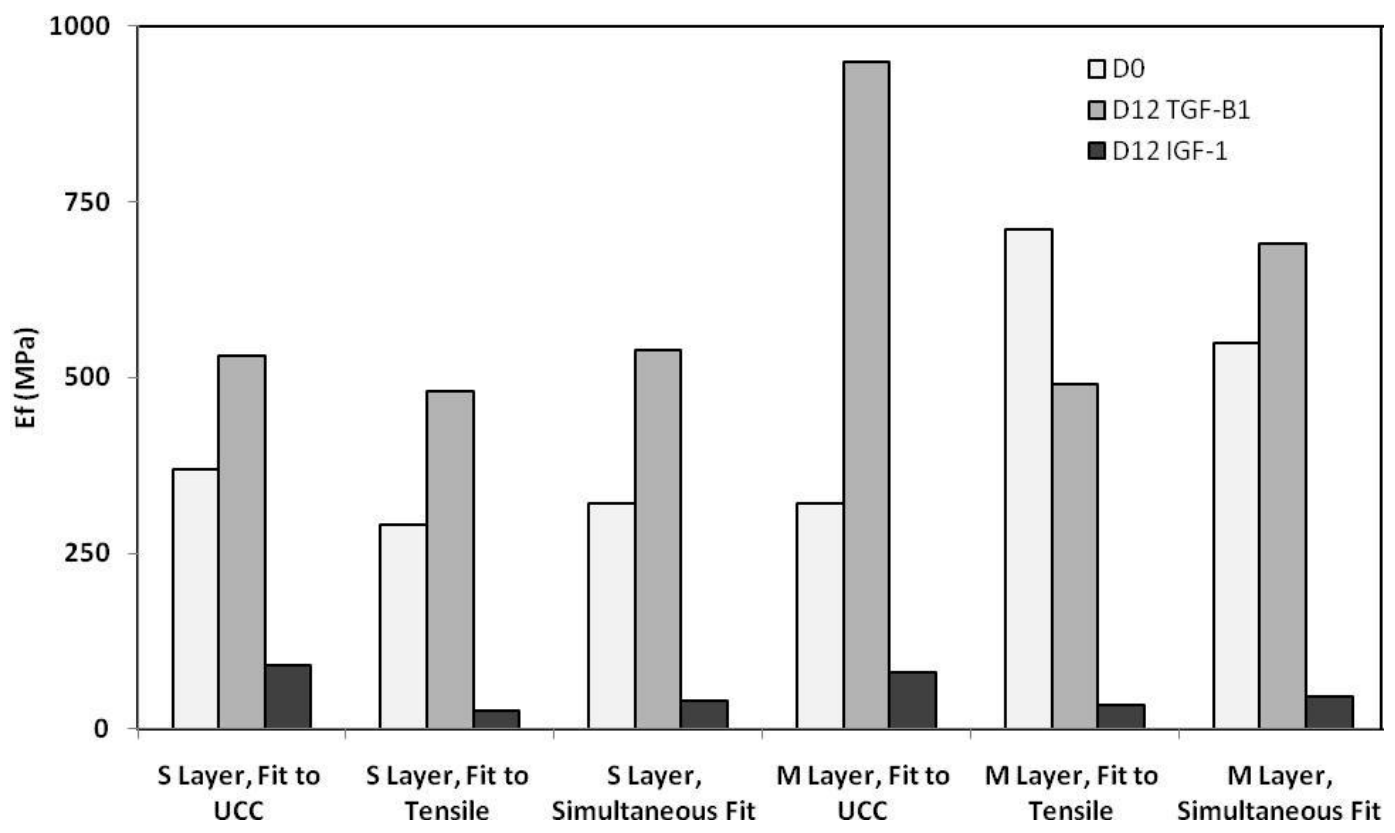

Figure 5.4. True $\mathrm{COL}$ fiber modulus $\mathrm{E}_{\mathrm{f}}$ determined for $\mathrm{S}$ and $\mathrm{M}$ Layer bovine newborn calf $A C$ before (D0) and after (D12 IGF-1, D12 TGF- $\beta 1$ ) in vitro growth. Numerical values are in Appendix B.5.5. Note that for all groups and fitting methods MAT shear modulus $\mu$, was determined to be $0.001 \mathrm{MPa}$. 


\subsubsection{Model Predictions}

Model predictions for $\mathrm{E}^{+}, \mathrm{E}^{-}, \mathrm{H}_{\mathrm{A}}$, and $v$ are shown in Figure 5.5, Figure

5.6, and Figure 5.7 for D0, D12 IGF-1 and D12 TGF- $\beta 1$ explants from both S and M layers. 


\section{D0, S Layer}
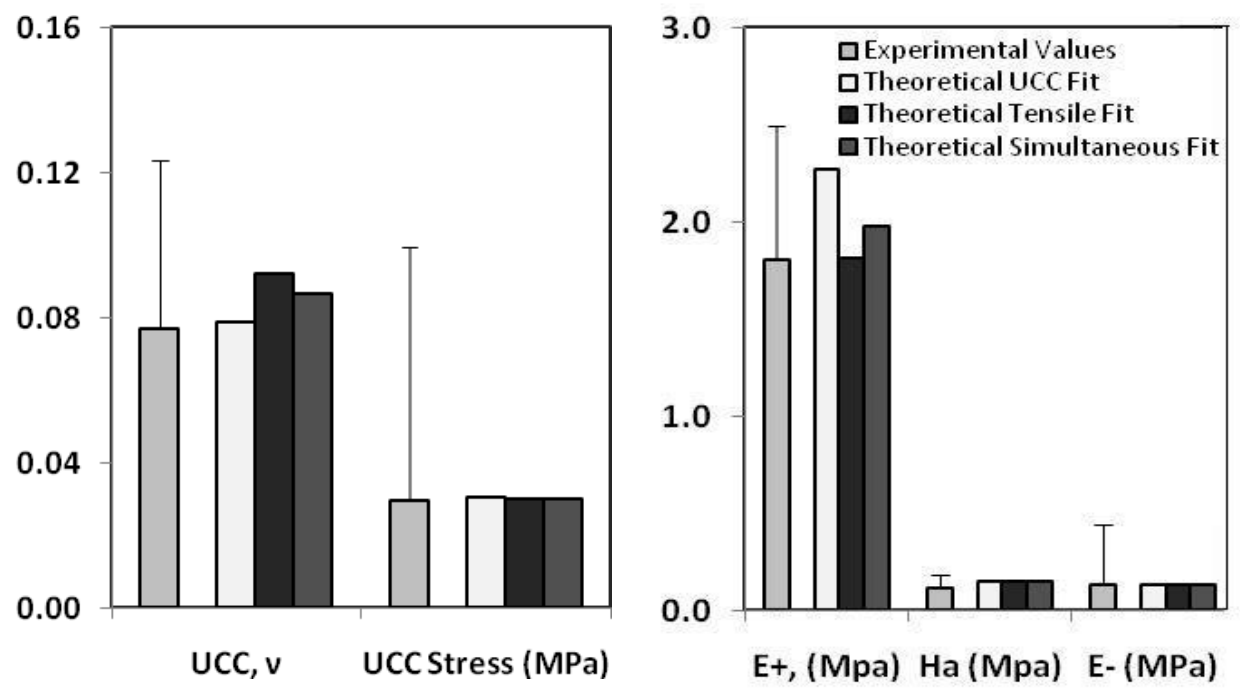

D0, M Layer
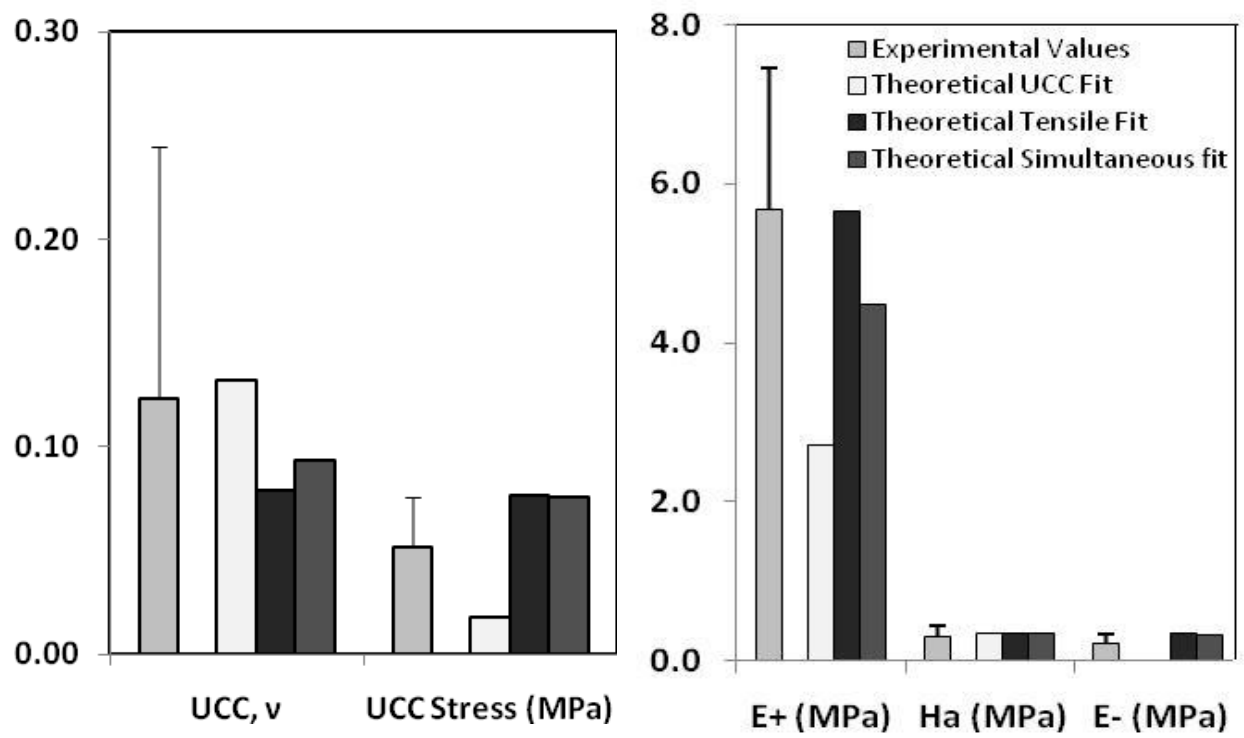

Figure 5.5. Experimental results compared with theoretical model predictions for $\mathrm{D} 0, \mathrm{~S}$ and $M$ layers. Experimental results are mean \pm SD. 
D12 TGF- $\beta 1$, S Layer
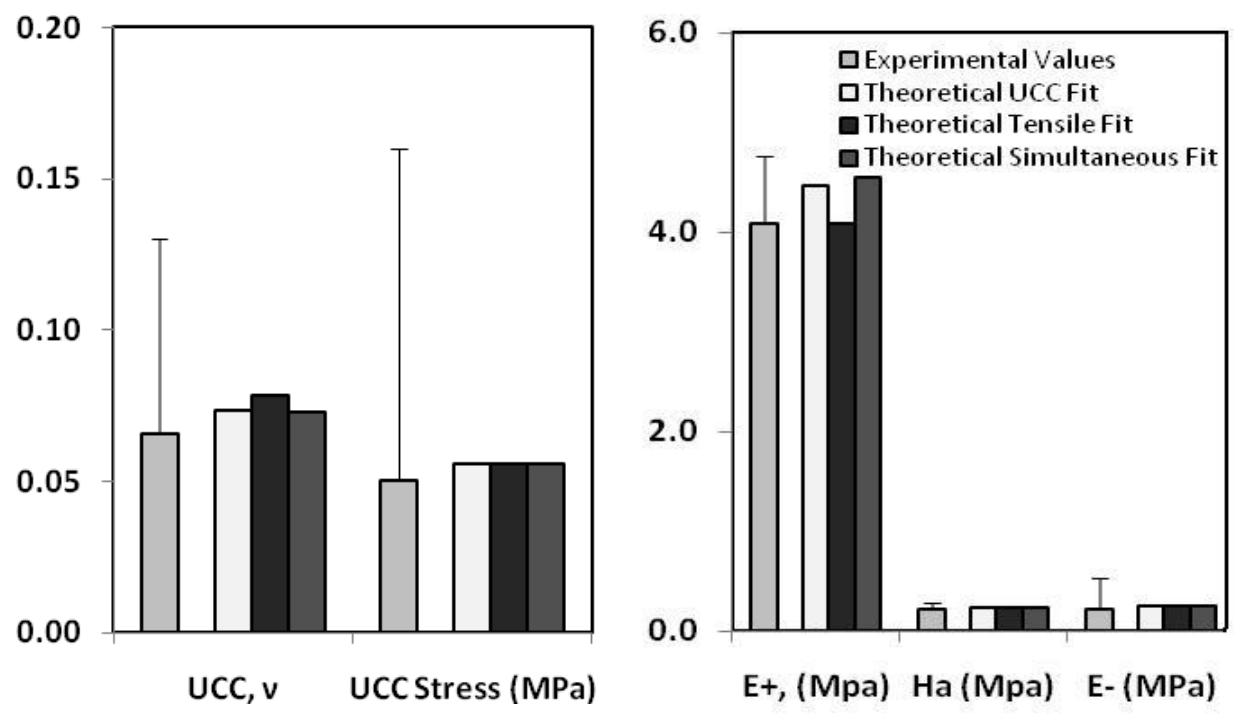

\section{D12 TGF- $\beta 1$, M Layer}
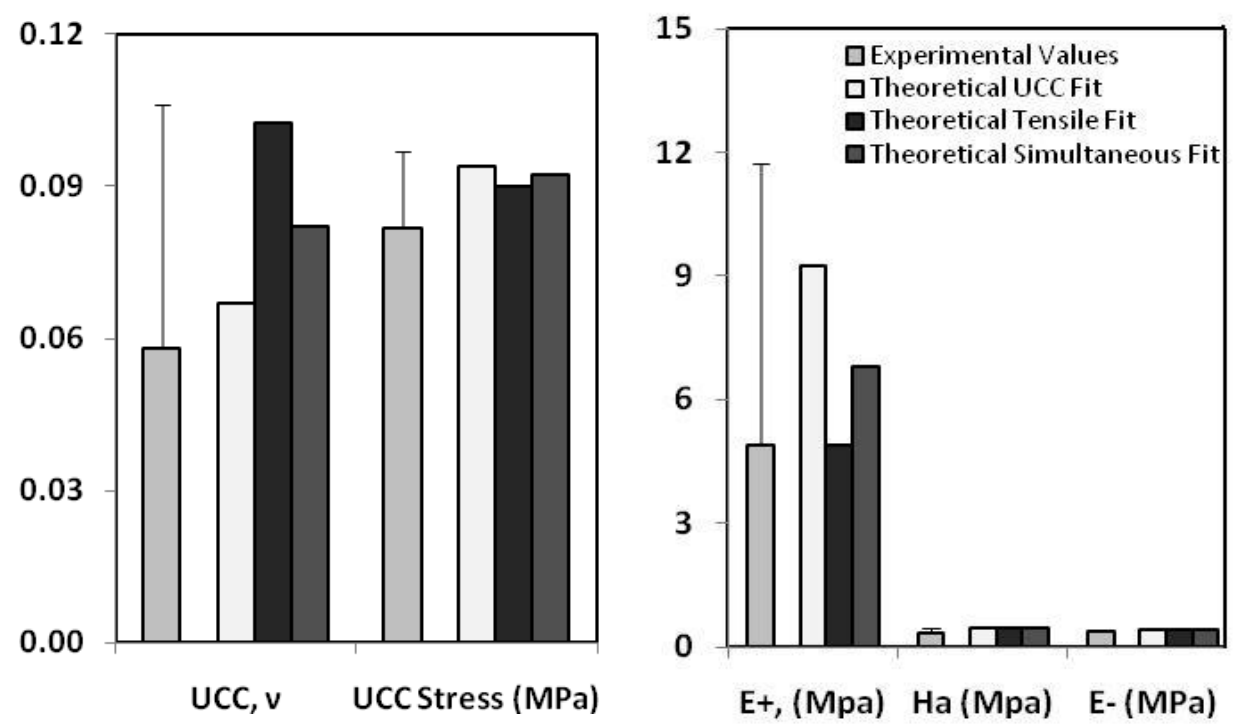

Figure 5.6. Experimental results compared with theoretical model predictions for D12 TGF- $\beta 1, S$ and M layers. Experimental results are mean \pm SD. 
D12 IGF-1, S Layer


D12 IGF-1, M Layer
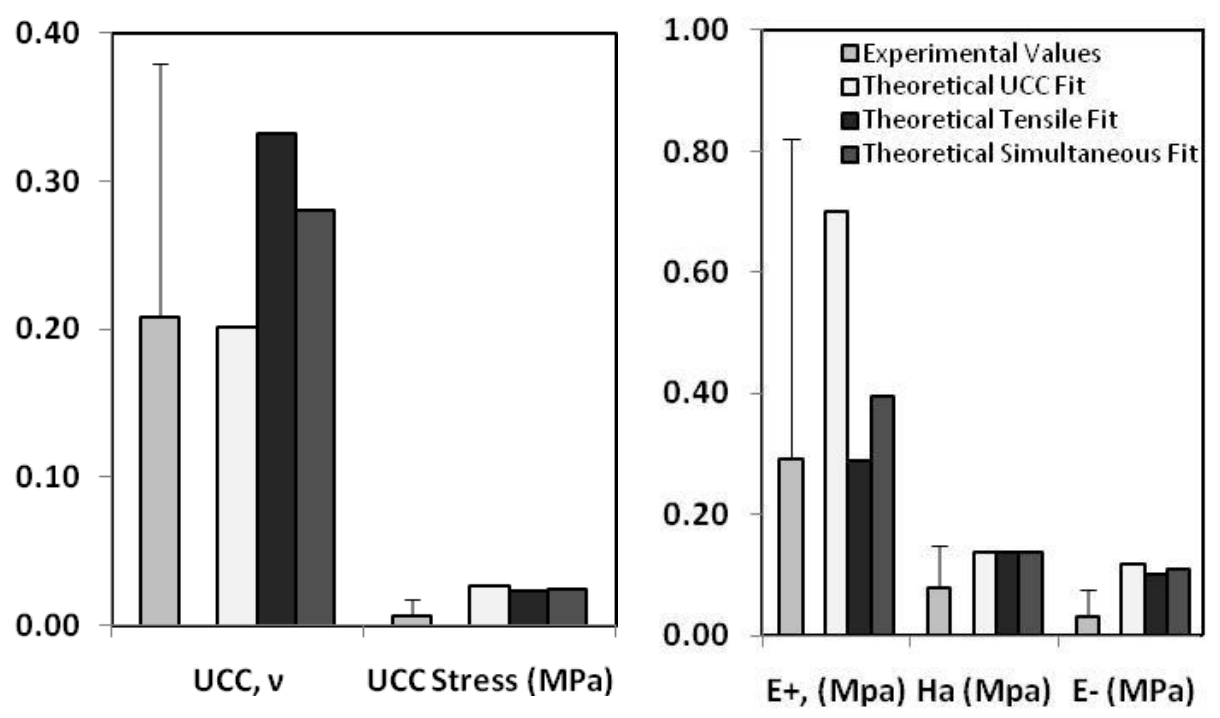

Figure 5.7. Experimental results compared with theoretical model predictions for D12 IGF-1, S and M layers. Experimental results are mean \pm SD. 
Table 5.2. Complete objective function values for Fit to UCC, Fit to Tensile and Simultaneous fit methods. The complete objective function was calculated using equation 5.2 .

\begin{tabular}{|c|c|c|c|}
\hline Group & Fit Method & $\begin{array}{c}\text { Complete Objective } \\
\text { Function, S Layer }\end{array}$ & $\begin{array}{c}\text { Complete Objective } \\
\text { Function, M Layer }\end{array}$ \\
\hline \multirow{3}{*}{ D0 } & Fit to UCC & 0.464 & 2.95 \\
\cline { 2 - 4 } & Fit to Tensile & 0.039 & 0.135 \\
\cline { 2 - 4 } & Simultaneous Fit & 0.180 & 1.196 \\
\hline \multirow{3}{*}{ D12 TGF- $\beta 1$} & Fit to UCC & 0.388 & 4.334 \\
\cline { 2 - 4 } & Fit to Tensile & 0.036 & 0.147 \\
\cline { 2 - 4 } & Simultaneous Fit & 0.464 & 1.894 \\
\hline \multirow{3}{*}{ D12 IGF-1 } & Fit to UCC & 0.546 & 0.422 \\
\cline { 2 - 4 } & Fit to Tensile & 0.217 & 0.154 \\
\cline { 2 - 4 } & Simultaneous Fit & 0.184 & 0.158 \\
\hline
\end{tabular}

\subsection{Discussion}

\subsubsection{Material Parameters}

The values determined for $\mathrm{E}_{\mathrm{f}}$ and $\mu$ for each culture group and layer provide insight into the constituent specific mechanical consequences of in vitro culture on AC. For all groups and fitting methods, $\mu$ was determined to be the lower bound of $0.001 \mathrm{MPa}$. This result suggests that the MAT constituent has a small or negligible contribution to the overall SM behavior during loading. It should be noted that the inclusion of the MAT constituent may provide a more physically comprehensive model. The MAT constituent also improved the stability and convergence rate of the FEA model. Due to the small determined $\mu$ values, 
mechanical properties were heavily dependent on the COL fiber modulus and the determined PG Cauchy stress function.

Previous studies have developed continuum level fibril reinforced material models to explain the mechanical behavior of $A C$ and other collagen fiber network reinforced soft tissues. Soulhat et al. (1999) proposed a similar nonstrain dependent COL fiber modulus, and fit the resulting model to dynamic UCC data for full thickness bovine humeral head AC samples. Additional studies have proposed elastic strain dependent (Korhonen et al. 2003; Lei and Szeri 2007) and strain dependent viscoelastic (Wilson et al. 2004; Wilson et al. 2005) COL fiber modulus models. For this study, a strain independent true COL fiber modulus is proposed. The effect of strain level on all experimental mechanical data $\left(\mathrm{E}^{+}, \mathrm{E}^{-}, \mathrm{v}\right.$, and $\left.\mathrm{H}_{\mathrm{A}}\right)$ was determined to not be significant $(\mathrm{p}>0.05)$ indicating that a strain independent COL fiber modulus model is appropriate. During the course of this study, a strain dependent COL material model was investigated, but did not provide a good fit to the experimental data used for this study. A comparison of COL fiber modulii from this study and other previous studies is shown below. 
Table 5.3. Tissue source and determined True COL fiber modulus for D0 explants from this and previous studies.

\begin{tabular}{|c|c|c|}
\hline Authors & Tissue Source & $\begin{array}{c}\mathbf{E}_{\mathrm{f}} \text { at equilibrium 7.5\% tensile } \\
\text { strain plus tare strain (Mpa) }\end{array}$ \\
\hline This Study & $\begin{array}{c}\text { D0 S Layer newborn } \\
\text { Bovine AC }\end{array}$ & 320 \\
\hline This Study & $\begin{array}{c}\text { D0 S Layer newborn } \\
\text { Bovine AC }\end{array}$ & 20 \\
\hline Korhonen et al. (2003) & $\begin{array}{c}\text { Lateral upper quadrant of } \\
\text { bovine patella }\end{array}$ & 513.8 \\
\hline Lei and Szeri (2007) & $\begin{array}{c}\text { 1-2 year old bovine Full } \\
\text { thickness plugs }\end{array}$ & 110 \\
\hline Soulhat et al. (1999) & $\begin{array}{c}\text { Full thickness bovine } \\
\text { humeral head }\end{array}$ & 0.4735 \\
\hline Wilson, et. al. 2004 & $\begin{array}{c}\text { Normal bovine patellar } \\
\text { cartilage }\end{array}$ & 0.2737 \\
\hline Wilson, et. al. 2005 & Bovine AC & \\
\hline
\end{tabular}

Note that for the models of Wilson et al. $(2004 ; 2005)$ the COL fiber modulus was highly dependent on viscoelastic effects rendering the effective COL modulus much lower at equilibrium compared to dynamic conditions.

The differences between $\mathrm{E}_{\mathrm{f}}$ for D0 and D12 groups suggests that the COL fiber network of newborn bovine calf $A C$ undergoes mechanical changes during in vitro culture. Since the AC model does account for the volume fraction of COL fibers in the tissue it can be deduced that the differences in $\mathrm{E}_{\mathrm{f}}$ are attributed to an intrinsic strengthening or weakening of COL fibers and/or the COL fiber network rather than COL fiber deposition. These results suggest that in vitro culture in TGF- $\beta 1$ results in increased COL fiber/COL network stiffness, while culture in IGF-1 results in weakening of COL fibers/COL network. 
A limitation of this study is the limited experimental data currently available. Since all experimental compressive data is from the medial or lateral ridges of the PFG tensile and explants should ideally all be from the same region. D12 tensile Modulii were scaled to ridge data according to the D0 ridge to groove relationships. Actual experimental tensile data for D12 ridge explants would be preferable for model validation and fitting. Additionally, reliable quantitative Poisson's ratio measurements in tension would provide an additional level of confidence and accuracy for the determined material parameters and model validation.

\subsubsection{Model Predictions}

Successful validation of the model through fitting to experimentally measured mechanical parameters is dependent on layer and culture. For example, for the DO S Layer group all three fitting methods (fit to UCC, fit to tension, and simultaneous fit) match all experimental parameters within one standard deviation of the experimental mean. However, the model does not fit as well to the IGF-1 S Layer group where some predictions are over a standard deviation from the mean. Generally, the model fits and predictions are much better for D0 and D12 TGF- $\beta 1$ groups vs. D12 IGF-1. This discontinuity across fit groups may suggest that the general weakening of D12 IGF-1 samples may be due to changes in the tissue during in vitro culture that are not accounted for in the model (i.e. not a change in $\mathrm{E}_{\mathrm{f}}$ or $\mu$ ). Also, model predictions fit better to $\mathrm{S}$ 
layer experimental data than $\mathrm{M}$ layer experimental data, which may be attributed to calcification of the M layer in newborn AC tissue.

The fitting methods used suggest that for complex models it is beneficial to validate the model using multiple methods (i.e. fit to UCC and predict tensile properties and fit to tensile properties and predict UCC properties). For example, the D12 IGF-1 layer fit to tensile results provide an excellent match to the experimental tensile modulus, but the UCC properties from the same fit are far from the experimental UCC values. Although the model is validated for a single tensile loading condition, it may or may not successful when used to predict alternative loading conditions depending on the experimental group.

This proposed constitutive model using an isotropic $\mathrm{COL}$ fiber distribution, a PB-cell model PG Cauchy stress equation, and a compressible Neo-Hookean ground substance matrix material provides an FEA implementable model for the equilibrium behavior of newborn bovine calf AC. This study is the first study to use a continuous fiber distribution model to predict $\mathrm{E}^{\mathrm{f}}$ using $\mathrm{CC}$, UCC, and tensile data from the same tissue source before and following in vitro growth. Furthermore, the model is able to capture the extreme tension-compression nonlinearity of $\mathrm{AC}$ for multiple groups. According to the complete objective function results in Table 5.2, validation of the model is generally better using the tensile fit to experimental data. Validation is also generally better for S layer compared to M layer and D0 and TGF- $\beta 1$ compared to IGF-1 according to the complete objective function results in Table 5.2. The Simultaneous fit method provides the best estimates of $E^{f}$ and $\mu$ for the experimental $A C$ groups used in this study. Further studies with different tissue sources and/or locations may lead to accurate complete joint models that could improve therapeutic strategies for 
treating cartilage defects and/or arthritis. These results as well as this FEA model may help to guide future AC tissue engineering studies and provide an insight into the complex constituent relationships that govern AC mechanical behavior. 


\section{List of References}

Asanbaeva A, Masuda K, Thonar EJ-MA, Klisch S M, Sah R L. "Regulation od immature cartilage growth by igf- 1 tgf-beta1, bmp-7 and pdgf-ab role of metabolic balance between fixed charge and collagen network." Biomech Model Mechanobiol., 2008: 7: 263-276.

Asanbaeva A, Tam J, Schumacher B L, Klisch S M, Masuda K, Sah R L. "Articular cartilage tensile integrity: Modulation by matrix depletion is maturationdependent." Archives of Biochemistry and Biophysics, 2008: 474: 175-182.

Ateshian G. "Anisotropy of Fibrous Tissues in Relation to the Distribution of Tensed and Buckled Fibers." J. Blomech. Eng, 2007: 129(2): 240-249.

Ateshian G A, Rajan V, Chahine N O, Canal C E, Hung C T. "Modeling the Matrix of Articular Cartilage Using a Continuous Fiber Angular Distribution Predicts Many Observed Phenomena." J. Biomech Eng., 2009: 131(6): 061003.

Atkin R J and Craine R E. "Continuum theories of mixtures: basic theory and historical development." Q J Mechanics Appl Math, 1976: 29(2) 209-244.

Baaijens F P T, Trickey W R, Laursen T A, Guilak F. "Large deformation finite element analysis of micropipette aspiration to determine the mechanical properties of the chondrocyte." Annals of Biomefical Engineering, 2005: 33 (4): 492-499.

Basser J P, Schneiderman R, Bank A R, Wachtel E, Maroudas A. "Mechanical Properties of the collagen network in human articular cartilage as measured by osmotic stress technique." Arch Biochem Biophys, 1998: 351(2) 207-219.

Bathe M, Rutledge G C, Grodzinsky A J, Tidor B. "Osmotis pressure of aqueous chondroitin sulfate solution: a molecular modeling investigation." Biophsical Journal, 2005: 89: 2357-2371.

Buckwalter A J, Mankin J H. "Articular cartilage repair: basic science and clinical progress. Areview of current status and prospects." Arthritis \& Rheumatism., 1998: 10(6) : 432-463.

Buschmann M, Grodzinsky A. "A Molecular Model of Proteoglycan-Associated Forces in Cartilage Mechanics." Journal of Biomechanical Engineering, 1995: 117: 179-192. 
Chahine N O, Chen H F, Hung C T, Ateshian G A. "Direct measurement of osmotic pressure of glycosaminoglycan solutions by membrane osmometry at room temperature." Biophysical Journal, 2005: 89: 1543-1550.

Charlebois M, McKee M D, Buschamann M D. "Nonlinear tensile properties of bovine articular cartialge and their variation with age and depth." J. Biomech. Eng., 2004: 126: 129-137.

Chen A C, Bae W C, Schingal R M, Sah R L. "Depth- and strain-dependent mechanical and electromechanical properties of full-thickness bovine articular cartialge in confined compression." Journal of Biomechanics, 2001: 34. 1-12.

Clark, J M. "The organisation of collagen fibrils in the superficial zones of articular cartialge ." Journal of Anatomy, 1990: 171: 117-130.

Comper W D, and Laurent T C. "Physiological fucntion of connective tissue polysaccharides." Physiological Reviews, 1978: 58: 255-315.

Davol A, Bingham M S, Sah R L, Klisch S M. "A nonlinear finite element model of cartilage growth." Biomechanics and Modeling in Mechanobiology, 2008: 7: 295307.

Ehrlich S, Wolff N, Schniderman R, Maroudas A, Parker K H, Winlove C P. "The Osmotic pressure of chondroitin sulphate solutions: Experimental measurements and theoretical analysis." Biorheology, 1998: 35 (6): 383-397.

Eisenberg S R, Grodzinsky A J. "Swelling of articular cartilage and other connective tissues: electromechanochemical forces." Journal of Orthopaedic Research, 1985: 3: 148-159.

Eyre, Davis. "Review: COllagen of Articular cartilage." Arthritis Research, 2001: 4 (1) 1-6.

Ficklin T P, Davol A, Klisch S M. "Simulating the growthe of articular cartialge explants in a permation bioreactor to aid in experimental protocol design." $\mathrm{J}$. Biomech Eng., 2009: 131(4) 041008.

Ficklin T, Thomas G, Barthel J C, Thonar E J, Masuda K, Asanbaeva A, Chen AC, Sah RL, Davol A, Klisch SM. "Articular cartilage mechanical and biochemical property relations before and after in vitro growth." Journal of Biomechanics, 2001: 40: 3967-3614.

Ficklin T, Thomas G, Barthel J C, Asanbaeva A, Thonar E J, Masuda K, Chen, A C, Sah R L, Davol A, Klisch, S M. "Articular cartilage mechanical and biochemical property relation before and after in vivo growth." Journal of Biomechanics, 2007: 40. 3607-3614. 
Fortin M, Soulhat J, Shirai-Adl A, Hunziker E B, Buschmann M D. "Unconfined compression of articular cartilage: nonlinear behavior and comparison with a fibril reinforced biphasic model." J. Biomech. Eng., 2000: 122 (2): 189-176.

Gasser C, Ogden R W, Holzpafel G A. "Hyperelastic modelling of arterial layers with distributed collagen fibre orientations." Journal of the Royal Society, 2006: 3(6): 15-35.

Grimaud E, Heymann D, Rédin F. "Recent advances in TGF- $\beta$ effects on chondrocyte metabolism: Potential therapeutic roles of TGF- $\beta$ in cartilage disorders." Cytokine \& Growth Factor Reviews, 2002: 13(3): 241-257.

Guilak F, Mow V C,. "The mechanical environment of the chondrocytr: a basic finite element model of cell-matrix interactions in articular cartilage." journal of Biomechanics, 2000: 33: 3-12.

Herbage D, Bouillet J, Bernago J-C. "Biochemical and physicochemical characterization of pepsin-solubilized type-ii collagen from bovine articular cartilage." J. Biochem, 1977: 161: 302-312.

Hochberg M C, Altman R D, Brandt K D, Clark B M, Dieppe P A, Griffin M R, Moskowitz R W, Schnitzer T J. "Guidelines for the Medical Management of Osteoarthritis." Arthritis and Rheumatisim, 1995: 38(11): 1541-1546.

Holzapfel, Gerhard A. "Determination of material models for arterial walls from uniaxial extension tests and histological structure." Journal of Theoretical Biology, 2006: 238: 290-302.

Huang C-Y, Stankiewicz A, Ateshian G A, Mow V C. "Anisotropy, inhomogeneity, andtension-compression nonlinearity ofAnisotropy, inhomogeneity, and tensioncompression nonlinearity of." Journal of Biomechanics, 2005: 38: 799-809.

Insall J M, Ranawat C S, Aglietti P, Shine J. "A comparicon of four models of total knee replacement prostheses." J Bone Joint Surg Am, 1976: 58: 754-765.

Julkunen P, livarinen J, Brama P, Arokoski J, Jurvelin J, Helminen H:

"Maturation of collagen fibril network structure in tibial and femoral cartilage of rabbits." Osteoarthritis and Cartilage, 2010: 18(3): 406-415.

Julkunen P, Kiviranta P, Wilson W, Jurvelin J S, Korhonen R K. "Characterization of articular cartilage by combining microscopic analysis with a fibril-reinforced finite-element model." Journal of Biomechanics, 2007: 40 (8): 18621870.

Kim, Y J, Sah, R L, Doong, J Y, Grodzinsky, A J. "Fluorometric assay of DNA in cartilage explants using hoechse 33258." Anal. Biochem., 1988: 174: 168-176. 
Klisch M S, Van Dyke T., Hoger A. "A theory of volumetric growth for compressible elastic materials." Mathematics and Mechanics of Solids, 2001: 6. $551-575$.

Klisch S M, Asanbaeva A, Oungoulian S R, Masuda K, Thonar E J-MA, Davol A, Sah R L. "A cartialge growth mixture model with collagen remodeling: validation protocols." J. Biomech Eng., 2008: 130(3): 031006.

Klisch, S M. "Bimodular polyconvex fiber-reinforced strain energy fuinctions for articular cartilage." J Biomech Eng, 2007: 129: 250-258.

Korhonen R K, Laasanen M S, Toyras J, Lappalainen R, Helminen H J, and Jurvelin J S,. "Fibril reinforced poroelastic model predicts specifically mechanical behavior of normal, proteoglycan depleted and collagen degraded articular cartilage." J Biomech, 2003: 36 (9) 1373-1379.

Kuettner, Klaus E. "Biochemistry of articular cartilage in health and disease." Clinical Biochemistry, 1992: 25(3): 155-163.

Lai W M, Hou J S, MowV C. "A Triphasic Theory for the Swelling and Deformation Behaviors of Articular Cartilage." J. Biomech Eng., 1991: 113(3): 245-259.

Lei F and Szeri AZ. " Predicting articular cartilage behavior with a non-linear microstructural model. ." Open Mech J, 2007: 1: p. 11-19.

Li L P, Cheung T M, Herzog W. "Three-dimensional fibril-reinforced finite element model of articular cartilage." Medical and biolobical engineering and computing, 2009: 47 (6) 607-615.

Lubliner, Jacob. Plasticity Theory (Revised Edition). New York: Dover Publications, 2008.

Maroudas, A. "Physiochemical properties of articular cartialge." Adult Articular Cartilage, 1979: 2: 215-290.

Marsden J E and Hughes T J R. Mathematical Foundations of Elasticity. Mineola, NY: Dover, 1994.

Mase T G, Semelser R E, Mase G E. Continuum Mechanics for Engineers, Third Edition. Boca Raton: CRC Press, Taylor Francis Group, 2009.

Mow CV, Guo EX. "Mechano-electrochemical properties of acrticular cartilage: Their inhomogeneities and Anisotropies." Annual Review of Biomedical Engineering, 2002: 4: 179-209.

Ogsten A G, Preston B N, Wells J D. "On the transport of compact particles through solutions of chain-polymers." Proc. R. Soc. Lond, 1973: 333: 297-316. 
Oungoulian R S, Chen S S, Davol A, Sah R L, Klisch S M. "Extended two compartmental swelling stress model and isotropic Cauchy stress equation for articular cartilage proteoglycans." Transactions of the ASME Summer Bioengineering Conference, , 2007.

Oungoulian, S R. A Polyconvex Strain Energy Function for Proteoglycan and Validation of a Growth Mixture Model with Collagen Remodeling [M.S]. M.S. Thesis, San Luis Obispo: California Polytechnic State Unversity, 2007.

Overbeek, T J. "The Donnan Equilibrium." Prog Biophys Chem, 1956: 6: 57-84.

Pal S, Tang L-H, Choi H, Habermann E, Rosenberg L, Roughley P, Poole A R. "Structural changes during development in bovine fetal epiphyseal cartilage." Collagen Rel. Res., 1981: 1: 151-176.

Pedregal, Pablo. Variational methods in nonlinear elasticity. Society for Industrial Mathematics, 1987.

Preston B N, Snowden J M, Houghton K T. "Model connective tissue systemsL The effect of proteoglycans on the distribution of small non-electrolytes and micro-ions." Biopolymers, 1972: 11: 1645-1659.

Rieppo J, Hyttinen M, Halmesmaki E, Ruotsalainen H, Vasara A, Kiviranta I, Jurvelin $\mathrm{J}$, Helminen $\mathrm{H}$. "Changes in spatial collagen content and collagen network architecture in porcine articular cartialge during growth and maturation." Osteoarthritis and Cartilage, 2009: 17 (4): 448-455.

Schwartz M H, Leo P H, Lewis J L. "A microscructural model for the elastic response of articular cartilage. ." J Biomech, 1994: 27(7): p. 865-873 .

Shinar H, Seo Y, Ikoma K, Kusaka Y, Eliav U, Navon G. "Mapping the fiber orientation in articular cartialge at rest and under pressure studied by $2 \mathrm{H}$ double quantum filtered MRI." Magnetic Resonance in Medicine, 2002: 48: 322-330.

Shirazi R, Vena P, Sah R L, Klisch S M. "Modeling collagen fibrils using a continuous volume fraction distribution function for use." Mathematics and Mechanics of Solids, 2010: In Press.

Silver FH, Bradica G, and Tria A,. "Elastic energy storage in human articular cartilage: Estimation of the elastic modulus for type II collagen and changes associated with osteoarthritis." Matrix Biology, 2002 : 21: 129-137.

SIMULIA. Abaqus 6.7 user subroutines reference manual. 2007.

Soltz M A, Ateshian, G A. "A conewise linear elasticity mixture model for the analysis of tension-compression nonlinearity in articular cartialge." J. Biomech Eng., 2000: 122(6): 576-586. . 
Soulhat J, Buschmann M D, Shirazi-Adl A. "A Fibril-Network-Reinforced Biphasic Model of Cartilage in Unconfined Compression." Journal of Biomechanical Engineering, 1999: 121 (3): 340-348.

Spencer, A.J. M. Continuum Mechanics. Mineola, N.Y.: Dover Publications, 1980.

Spilker R L, Suh J K, Mow V C,. "A finite element analysis of the indentation stress relaxation sesponse of linear biphasic articular cartilage." Journal of Biomechanical Engineering, 1992: 144:144-2.

Stender M. E., Balcom N., Berg-Johansen B., Dills K. J., Dyk D, Sah R. L., Klisch S. M., Hazelwood S. J.:. "Differential Regulation of Articular Cartilage Tensil Properties by IGF-1 and TGF-beta1 duting in vitro growth." Submitted to: International Conference on the Mechanics of Biomaterials and Tissues, 2011.

Stewart, K M. Mechanical simulation of articular cartialge [M.S.]. San Luis Obispo: California Polytechnic State University: 2009, 2007.

Sun D D, Guo X E, Likhitpanichkul M, Lai W M, Mow V C. "The Influence of the Fixed Negative Charges on Mechanical and Electrical Behaviors of Articular Cartilage Under Unconfined Compression." J. Biomech Eng., 2004: 126(1): 6-16.

Thomas G C, Asanbaeva A, Vena P, Sah R L, Klisch S M. "A nonlinear constituent based viscoelastic model for articular cartilage and analysis of tissue remodeling due to altered glycosaminoglycan-collagen interactions." J. Blomech Eng., 2009: 131:101002.

Van Turnhout M C, Schipper H, Engel B, Buist W, Kranenbarg S, Leeuwen. "Postnatal development of collagen structure in bovine articular cartialge." BMC Developmental Biology, 2010: 10: 62.

Van Turnhout MC, Haazelager MB, Gijsen MA, Schipper H, Kranenbarg S, Van Leeuwen JL. "Quantitativedescription of collagen structure in the articular cartilage of the young and adult equine distal metacarpus." Animal Biology, 2008: 58(4): 353-370.

Veress A I, Weiss J A, Gullberg G T, Vince D G, Rabbitt R D. "Strain measurement in coronary arteries using intravascular ultrasound and deformable images." Journal of biomechanical engineering, 2002: 124 (6) 734-742.

Wang C C-B, Chahine N O, Hung C T, Ateshian G A. "Optical Determination of Anisotropic Material Properties of Bovine Articular Cartilage in Compression." $J$. Biomech, 2003: 36(3): 339-353.

Williams G M, Dills K J, Flores C R, Stender M E, Stewart K M, Nelson L M, Chen A C, Masuda K, Hazelwood S J, Klissch S M, Sah R L. "Differential regulation of immature articular cartilage compressive modulii and poisson's 
ratios by in vitro stimulation with IGF-1 and TGF- B1." Journal of Biomechanics, 2010: 43: 2501-2507.

Williams G M, Chan E F, Temple-Wong M M, Bae W C, Masuda K, Bugbee W D, Sah R L. "Shape, Loading, and motion in the bioengineering design, fabrication, and testing of personalized synovial joints." Journal of Biomechanics, 2009: ePub: 10.6.2009.

Williams R P, Comper W D. "Osmotic flow casued by polyelectrolytes." Biophysical Chemistry, 1990: 36: 223-234.

Williamson A K, Chen A C, Sah R L. "Compressive properties and functioncomposition relationships of developing bovine articular cartilage." Journal of Orthopaedic Research, 2001: 1113-1121.

Williamson A K, Chen A C, Masuda K, Thonar E J, Sah R L "Tensile mechanical properties of bovine articular cartilage variations with growth and relationships to collagen network components." Journal of Orthopedic Research, 2003: 21. 872880 .

Wilson W, van Donkelaar C C, van Rietbergen B, Ito K, and Huiskes R. "Stresses in the local collagen network of articular cartilage: A poroviscoelastic fibrilreinforced finite element study." J Biomech, 2004: 37: 357-66.

Wilson W, van Donkelaar CC, van Rietbergen B, Huiskes R. "A fibril-reinforced poroviscoelastic swelling model for articular cartilage." J Biomech, 2005: 38(6): p. 1195-204.

Yelin, E, Cisternas M, Foreman A, Pasta D, Murphy L, Helmick C. "National and state medical expenditures and lost earnings attributable to arthritis and other rheumatic conditions." Morbidity and Mortality Weekly Proprt, 2007: 56(1): 4-7.

Zambrano NZ, Montes GS, Shigihara KM, Sanchez EM, Junqueira LC. "Collagen arrangement in cartialges." Acta Anatomica, 1982: 113: 26-38. 


\section{Appendix A: Derivations}

\section{A.1.1 Derivative of the Determinant of a Second Order Tensor}

This derivation is necessary to derive the MAT Second Piola-Kirchoff stress tensor.

Let $\mathbf{A}$ be a second order tensor with $f(\mathbf{A})=\operatorname{det} \mathbf{A}$. From the definition of the derivative of a scalar valued function of a tensor where $\mathbf{B}$ is an arbitrary second order tensor ${ }^{4}$.

$$
\begin{gathered}
\frac{\partial f}{\partial \mathbf{A}}: \mathbf{B}=\frac{\partial}{\partial \alpha} \operatorname{det}(\mathbf{A}+\alpha \mathbf{B}) \\
\frac{\partial f}{\partial \mathbf{A}}: \mathbf{B}=\left.\frac{\partial}{\partial \alpha} \alpha \mathbf{A}\left(\frac{1}{\alpha} \mathbf{I}+\mathbf{A}^{-1} \mathbf{B}\right)\right|_{\alpha=0} \\
\frac{\partial f}{\partial \mathbf{A}}: \mathbf{B}=\left.\frac{\partial}{\partial \alpha}\left[\alpha^{3} \operatorname{det}(\mathbf{A}) \operatorname{det}\left(\frac{1}{\alpha} \mathbf{I}+\mathbf{A}^{-1} \mathbf{B}\right)\right]\right|_{\alpha=0}
\end{gathered}
$$

Expand the determinant of a tensor in terms of the invariants of the tensor

$$
\operatorname{det}(\lambda I+A)=\lambda^{3}+I_{1}(\text { A }) \lambda^{2}+I_{2}(\text { A }) \lambda+I_{3}(\text { A })
$$

Applying the expansion of the determinant of a tensor in terms of the tensors invariants

$$
\begin{gathered}
\frac{\partial f}{\partial \mathbf{A}}: \mathbf{B}=\left.\frac{\partial}{\partial \alpha}\left[\alpha^{3} \operatorname{det}(\mathbf{A})\left(\frac{1}{\alpha^{3}}+I_{1}\left(\mathbf{A}^{-1} \mathbf{B}\right) \frac{1}{\alpha^{2}}+I_{2}\left(\mathbf{A}^{-1} \mathbf{B}\right) \frac{1}{\alpha}+I_{3}\left(\mathbf{A}^{-1} \mathbf{B}\right)\right)\right]\right|_{\alpha=0} \\
\frac{\partial f}{\partial \mathbf{A}}: \mathbf{B}=\left.\operatorname{det}(\mathbf{A}) \frac{\partial}{\partial \alpha}\left[\mathbf{I}+I_{1}\left(\mathbf{A}^{-1} \mathbf{B}\right) \alpha+I_{2}\left(\mathbf{A}^{-1} \mathbf{B}\right) \alpha^{2}+I_{3}\left(\mathbf{A}^{-1} \mathbf{B}\right) \alpha^{3}\right]\right|_{\alpha=0}
\end{gathered}
$$

\footnotetext{
${ }^{4}$ Note that this derivation is reproduced from Marsden and Hughes (1994)
} 


$$
\begin{gathered}
\frac{\partial f}{\partial \mathbf{A}}: \mathbf{B}=\left.\operatorname{det}(\mathbf{A}) \frac{\partial}{\partial \alpha}\left[I_{1}\left(\mathbf{A}^{-1} \mathbf{B}\right)+2 I_{2}\left(\mathbf{A}^{-1} \mathbf{B}\right) \alpha+3 I_{3}\left(\mathbf{A}^{-1} \mathbf{B}\right) \alpha^{2}\right]\right|_{\alpha=0} \\
\frac{\partial f}{\partial \mathbf{A}}: \mathbf{B}=\operatorname{det}(\mathbf{A}) I_{1}\left(\mathbf{A}^{-1} \mathbf{B}\right)
\end{gathered}
$$

Recall $I_{1}(\mathbf{A})=\operatorname{tr}(\mathbf{A})$ and

$$
\frac{\partial f}{\partial \mathbf{A}}: \mathbf{B}=\operatorname{det}(\mathbf{A}) \operatorname{tr}\left(\mathbf{A}^{-1} \mathbf{B}\right)=\operatorname{det}(\mathbf{A}) \mathbf{A}^{-T_{:}} \mathbf{B}
$$

And because $B$ is arbitrary and $f=\operatorname{det} A$

$$
\frac{\partial \operatorname{det} \mathbf{A}}{\partial \mathbf{A}}=\operatorname{det}(\mathbf{A}) \mathbf{A}^{-\mathbf{T}}
$$

If $\mathbf{A}$ is invertible and symmetric

$$
\frac{\partial \operatorname{det} \mathbf{A}}{\partial \mathbf{A}}=\operatorname{det}(\mathbf{A}) \mathbf{A}^{-1}
$$

Or alternatively,

$$
\frac{\partial \operatorname{det} \mathbf{A}}{\partial \mathbf{A}_{\mathrm{AB}}}=\operatorname{det}(\mathbf{A}) \mathbf{A}_{\mathrm{BA}}^{-1}
$$

\section{A.1.2 Partial derivative of a Tensor With respect to its Inverse}

This derivation is necessary to calculate the MAT elasticity tensor.

The partial derivative of a tensor with respect to its inverse, $\frac{\partial \mathrm{c}_{A B}^{-1}}{\partial \mathrm{c}_{C D}}$.

$$
\begin{gathered}
\mathrm{C}_{\mathrm{AM}}^{-1} \mathrm{C}_{\mathrm{MN}}=\delta_{\mathrm{AN}} \\
\frac{\partial}{\partial C_{C D}}\left(\mathrm{C}_{\mathrm{AM}}^{-1} \mathrm{C}_{\mathrm{MN}}\right)=0 \\
\frac{\partial C_{A M}^{-1}}{\partial \mathrm{C}_{C D}} C_{M N}+C_{A M}^{-1} \frac{\partial C_{M N}}{\partial C_{C D}}=0
\end{gathered}
$$




$$
\frac{\partial C_{A M}^{-1}}{\partial \mathrm{C}_{C D}} C_{M N}=-\frac{1}{2} C_{A M}^{-1}\left(\delta_{M C} \delta_{N D}+\delta_{M D} \delta_{N C}\right)
$$

Multiplying both sides by $C_{N B}^{-1}$ gives:

$$
\begin{gathered}
\frac{\partial C_{A M}^{-1}}{\partial \mathrm{C}_{C D}} C_{M N} C_{N B}^{-1}=-\frac{1}{2} C_{A M}^{-1} C_{N B}^{-1}\left(\delta_{M C} \delta_{N D}+\delta_{M D} \delta_{N C}\right) \\
\frac{\partial C_{A M}^{-1}}{\partial \mathrm{C}_{C D}} \delta_{M B}=-\frac{1}{2}\left(C_{A C} C_{B D}+C_{A D} C_{B C}\right) \\
\frac{\partial C_{A B}^{-1}}{\partial \mathrm{C}_{C D}}=-\frac{1}{2}\left(C_{A C} C_{B D}+C_{A D} C_{B C}\right)
\end{gathered}
$$

\section{A.1.3 Polyconvexity}

Polyconvexity is used as a means of ensuring material stability and numerical convergence in FEA models.

\section{Ground Matrix Strain Energy Density Function}

Given:

$$
\begin{gathered}
W^{M A T}=\frac{1}{2} \mu[(\operatorname{tr}(\mathbf{C})-3)-\ln (\operatorname{det}(\mathbf{C}))]=\frac{1}{2} \mu\left[\mathrm{F}_{\mathrm{mA}} \mathrm{F}_{\mathrm{mA}}-\ln \left([\operatorname{det}(\mathbf{F})]^{2}\right)\right] \\
W^{M A T}=\left[\frac{1}{2} \mu \mathrm{F}_{\mathrm{mA}} \mathrm{F}_{\mathrm{mA}}\right]+[-\mu \ln (\operatorname{det}(\mathbf{F}))]=W^{\mathrm{MAT}-1}(\mathbf{F})+W^{\mathrm{MAT}-2}(\operatorname{det}(\mathbf{F}))
\end{gathered}
$$

If $W^{\text {MAT-1 }}(\mathbf{F})$ is a convex function of $\mathbf{F}$ and $W^{\text {MAT-2 }}(\operatorname{det}(\mathbf{F}))$ is a convex function of $\operatorname{det}(\mathbf{F})$, then $\mathrm{W}^{\mathrm{MAT}}(\mathbf{F})$ is polyconvex.

$$
\frac{\partial W^{\mathrm{MAT}-1}}{\partial \mathrm{F}_{\mathrm{ip}}}=\frac{\partial}{\partial \mathrm{F}_{\mathrm{ip}}}\left[\frac{1}{2} \mu\left[\mathrm{F}_{\mathrm{mA}} \mathrm{F}_{\mathrm{mA}}\right]\right]=\mu \mathrm{F}_{\mathrm{mA}} \frac{\partial}{\partial \mathrm{F}_{\mathrm{ip}}}\left[\mathrm{F}_{\mathrm{mA}}\right]=\mu \mathrm{F}_{\mathrm{mA}}\left[\delta_{\mathrm{mi}} \delta_{\mathrm{AP}}\right]=\mu \mathrm{F}_{\mathrm{ip}}
$$

And 


$$
\frac{\partial^{2} \mathrm{~W}^{\mathrm{MAT}-1}}{\partial \mathrm{F}_{\mathrm{iP}} \partial \mathrm{F}_{\mathrm{jQ}}}=\frac{\partial}{\partial \mathrm{F}_{\mathrm{jQ}}}\left[\mu \mathrm{F}_{\mathrm{ip}}\right]=\mu\left[\delta_{\mathrm{ij}} \delta_{\mathrm{PQ}}\right]
$$

Similarly,

$$
\frac{\partial^{2} W^{\operatorname{MAT}-2}}{\partial \operatorname{det}(\mathbf{F}) \partial \operatorname{det}(\mathbf{F})}=\frac{\partial}{\partial \operatorname{det}(\mathbf{F})}\left[\frac{\partial}{\partial \operatorname{det}(\mathbf{F})}[-\mu \ln (\operatorname{det}(\mathbf{F}))]\right]=\frac{\partial}{\partial \operatorname{det}(\mathbf{F})}\left[-\mu \frac{1}{\operatorname{det}(\mathbf{F})}\right]=2 \mu \frac{1}{[\operatorname{det}(\mathbf{F})]^{2}}
$$

For both $W^{\mathrm{MAT}-1}$ and $W^{\mathrm{MAT}-2}$ if $\mu>0$, then $\frac{\partial^{2} W^{P^{L}}}{\partial \mathrm{F} \partial \mathrm{F}}>0$ and the strain energy function is polyconvex.

\section{Collagen Strain Energy Density Function}

Given the proposed true strain energy function for pure collagen fibrils with no pre-strain:

$$
\psi^{\mathrm{COL}}=\frac{1}{2}\left(\mathrm{E}_{\mathbf{n}}\right)^{2} \mathrm{E}_{\mathrm{f}}
$$

where $\mathrm{E}_{\mathbf{n}}$ is one dimensional Green-Lagrangian strain

$$
\frac{\partial \psi^{\mathrm{COL}}}{\partial \mathbf{F}}=\mathrm{E}_{\mathrm{f}} \mathrm{E}_{\mathbf{n}} \frac{\partial \mathrm{E}_{\mathbf{n}}}{\partial \mathbf{F}}
$$

Calculating the second derivative,

$$
\frac{\partial^{2} \psi^{\mathrm{COL}}}{\partial \mathbf{F} \partial \mathbf{F}}=\mathrm{E}_{\mathrm{f}} \mathrm{E}_{\mathbf{n}} \frac{\partial^{2} \mathrm{E}_{\mathbf{n}}}{\partial \mathbf{F} \partial \mathbf{F}}+\mathrm{E}_{\mathrm{f}} \frac{\partial \mathrm{E}_{\mathbf{n}}}{\partial \mathbf{F}} \frac{\partial \mathrm{E}_{\mathbf{n}}}{\partial \mathbf{F}}
$$

From Shirazi et. Al. (Shirazi et al. 2010) the $\frac{\partial^{2} E_{n}}{\partial F \partial F}$ and $\frac{\partial E_{n}}{\partial F} \frac{\partial E_{n}}{\partial F}$ terms are shown to be positive. It is noted that for $\mathrm{E}_{\mathbf{n}}>0$ the Heaviside step function and consequently the COL strain energy density will be zero. Therefore the OCL strain energy density is polyconvex provided $\mathrm{E}_{\mathrm{f}}>0$ 


\section{Proteoglycan Strain Energy Density Function}

For proof of Polyconvexity for the proteoglycan constituent please refer to Appendix A.4.4.

\section{A.1.4 Hemholtz Free Energy Equation}

The result of this derivation is integrated with respect to the determinant of the deformation gradient tensor to obtain the PG strain energy density function, as shown in appendix A.4.1.

The Cauchy stress for a mixture of $v-1$ elastic growing materials and an inviscid fluid is given in [3] as $^{5}$ :

$$
\mathbf{T}^{\alpha}=\sum_{\beta=1}^{v} \rho^{\beta} \frac{\partial \Psi^{\beta}}{\partial \mathbf{F}^{\alpha}}\left(\mathbf{F}^{\alpha}\right)^{\mathrm{T}}
$$

Assuming process reversibility and neglecting changes in chemical potential energy, so that Hemholtz free energy reduces to a function of strain energy $\Psi=\frac{\mathrm{W}}{\mathrm{P}_{\mathrm{o}}}$

$$
\mathbf{T}^{\alpha}=\sum_{\beta=1}^{v} \frac{\rho^{\beta}}{\rho_{0}^{\beta}} \frac{\partial \Psi^{\beta}}{\partial \mathbf{F}^{\alpha}}\left(\mathbf{F}^{\alpha}\right)^{\mathbf{T}}
$$

Assuming that the constituent strain energy functions, $W^{\beta}$ depend only on the respective constituent deformation gradients, $\mathbf{F}^{\beta}$

\footnotetext{
${ }^{5}$ Note that this derivation is reproduced from Oungoulian S R (2007)
} 


$$
\mathbf{T}^{\alpha}=\frac{\rho^{\alpha}}{\rho_{0}^{\alpha}} \frac{\partial \Psi^{\alpha}}{\partial \mathbf{F}^{\alpha}}\left(\mathbf{F}^{\alpha}\right)^{\mathrm{T}}
$$

Assuming for the GAG constituent $(\alpha=\mathrm{GAG})$ an isotropic strain energy function that depends only on $\mathbf{F}^{\mathrm{GAG}}$. Then, applying the chain rule

$$
\mathbf{T}^{\mathrm{GAG}}=\frac{\rho^{\mathrm{GAG}}}{\rho_{0}^{\mathrm{GAG}}} \frac{\partial W^{\mathrm{GAG}}}{\partial \mathrm{j}^{\mathrm{GAG}}} \frac{\partial \mathrm{J}^{\mathrm{GAG}}}{\partial \mathbf{F}^{\mathrm{GAG}}}\left(\mathbf{F}^{\mathrm{GAG}}\right)^{\mathrm{T}}
$$

Applying the formula from Appendix A.1.1

$$
\mathbf{T}^{\mathrm{GAG}}=\frac{\rho^{\mathrm{GAG}}}{\rho_{0}^{\mathrm{GAG}}} \frac{\partial W^{\mathrm{GAG}}}{\partial j^{\mathrm{GAG}}} \mathrm{J}^{\mathrm{GAG}} \mathbf{]}
$$

Recalling that from continuity, $J=\frac{\rho_{0}}{\rho}$

$$
\mathbf{T}^{\mathrm{GAG}}=\frac{\partial \mathrm{W}^{\mathrm{GAG}}}{\partial \mathrm{j}^{\mathrm{GAG}}} \mathbf{I}
$$

\section{A.1.5 Confined and Unconfined Compression Constituent Stresses}

This appendix outlines the process used to calculate constituent stresses for CC and UCC loading consitions from Chapter 4.

Recall the proteoglycan stress equation.

$$
\mathbf{T}^{\mathrm{PG}}=-\alpha\left(\frac{\rho_{0}^{P G}}{J}\right)^{2} \mathbf{I}
$$

Where for confined compression at applied strain $\varepsilon$,

$$
\mathrm{I}=1+\varepsilon
$$


And for unconfined compression and tension assuming an isotropic material $\left(v_{31}=v_{32}=v\right.$ for 3-direction loading $)$

$$
I=\left(1+\varepsilon_{\text {applied }}\right)\left(1+v \varepsilon_{\text {transverse }}\right)^{2}
$$

Solid matrix Stress can be calculated using experimentally measured equilibrium confined compression modulus and strain $\mathrm{H}_{\mathrm{A}}$ and $\varepsilon$, respectively.

$$
\mathbf{T}^{\mathrm{SM}}=\mathrm{H}_{\mathrm{A}} * \varepsilon \mathbf{I}
$$

Using the stress balance hypothesis:

$$
\mathbf{T}^{\mathrm{COL}+\mathrm{MAT}}=\mathbf{T}^{\mathrm{SM}}-\mathbf{T}^{\mathrm{PG}}
$$

\section{A.4.1 PG Cauchy Stress}

Given:

$$
\mathbf{T}^{\mathrm{PG}}=\frac{\partial \mathrm{W}^{\mathrm{PG}}}{\partial \mathrm{J}^{\mathrm{PG}}} \mathbf{I}
$$

Calculating the derivative of PG strain energy

$$
\mathbf{T}^{\mathrm{GAG}}=-\alpha\left(\frac{\rho_{0}^{\mathrm{GAG}}}{J}\right)^{2.5} \mathbf{I}
$$

\section{A.4.2 PG Material Elasticity Tensor}

Starting with the Cauchy stress 


$$
\mathbf{T}^{\mathrm{GAG}}=-\alpha\left(\frac{\rho_{0}^{\mathrm{GAG}}}{J}\right)^{2.5} \mathbf{I}
$$

And applying the stress transformation relationship the Second Piola Kirchoff stress is

$$
\mathrm{S}_{\mathrm{AB}}^{\mathrm{GAG}}=\left(\frac{-\alpha\left(\rho_{0}^{\mathrm{GAG}}\right)^{2.5}}{\left(\operatorname{det}\left(\mathrm{C}^{\mathrm{GAG}}\right)\right)^{1.25}}\right) \mathrm{C}_{\mathrm{AB}}^{-1}
$$

And the definition

$$
\widetilde{\mathbb{C}}_{\mathrm{ABCD}}^{\mathrm{GAG}}=2 \frac{\partial \mathrm{S}_{\mathrm{AB}}^{\mathrm{GAG}}}{\partial \mathrm{C}_{\mathrm{CD}}}
$$

Apply the product rule

$$
\widetilde{\mathbb{C}}_{\mathrm{ABCD}}^{\mathrm{GAG}}=2\left[\left(\frac{-\alpha\left(\rho_{0}^{\mathrm{GAG}}\right)^{2.5}}{\left(\operatorname{det}\left(\mathrm{C}^{\mathrm{GAG}}\right)\right)^{1.25}}\right) \frac{\partial \mathrm{C}_{\mathrm{AB}}^{-1}}{\partial \mathrm{C}_{\mathrm{CD}}}+\frac{\partial}{\partial \mathrm{C}_{\mathrm{CD}}}\left(\frac{-\alpha\left(\rho_{0}^{\mathrm{GAG}}\right)^{2.5}}{\left(\operatorname{det}\left(\mathrm{C}^{\mathrm{GAG}}\right)\right)^{1.25}}\right) \mathrm{C}_{\mathrm{AB}}^{-1}\right]
$$

Applying the result from appendix A.1.3

$$
\mathbb{C}_{\mathrm{ABCD}}^{\mathrm{GAG}}=\left(\frac{\alpha\left(\rho_{0}^{\mathrm{GAG}}\right)^{2.5}}{\left(\operatorname{det}\left(\mathrm{C}^{\mathrm{GAG}}\right)\right)^{1.25}}\right)\left(C_{A C} C_{B D}+C_{A D} C_{B C}\right)+\frac{\partial}{\partial \mathrm{C}_{\mathrm{CD}}}\left(\frac{-2 \alpha\left(\rho_{0}^{\mathrm{GAG}}\right)^{2.5}}{\left(\operatorname{det}\left(\mathrm{C}^{\mathrm{GAG}}\right)\right)^{1.25}}\right) \mathrm{C}_{\mathrm{AB}}^{-1}
$$

Modifying the second and fourth terms as follows:

$$
\frac{\partial}{\partial \mathrm{C}_{\mathrm{CD}}}\left(\frac{-2 \alpha\left(\rho_{0}^{\mathrm{GAG}}\right)^{2.5}}{\left(\operatorname{det}\left(\mathrm{C}^{\mathrm{GAG}}\right)\right)^{1.25}}\right) \mathrm{C}_{\mathrm{AB}}^{-1}=-2 \alpha\left(\rho_{0}^{\mathrm{GAG}}\right)^{2.5} \frac{\partial}{\partial \mathrm{C}_{\mathrm{CD}}}\left(\operatorname{det}\left(\mathrm{c}^{\mathrm{GAG}}\right)^{-1.25}\right) \mathrm{C}_{\mathrm{AB}}^{-1}
$$

Apply the chain rule

$$
=1.25 \frac{2 \alpha\left(\rho_{0}^{\mathrm{GAG}}\right)^{2.5} \frac{\partial}{\partial \mathrm{C}_{\mathrm{CD}}}\left(\operatorname{det}\left(\mathrm{c}^{\mathrm{GAG}}\right)\right)}{\left(\operatorname{det}\left(\mathrm{c}^{\mathrm{GAG}}\right)\right)^{2.25}} \mathrm{C}_{\mathrm{AB}}^{-1}
$$

Apply Jacobi's formula in Appendix A1.1 


$$
=2.5 \frac{\alpha\left(\rho_{0}^{\mathrm{GAG}}\right)^{2.5}\left(\operatorname{det}\left(\mathrm{c}^{\mathrm{GAC}}\right)\right) \mathrm{C}_{\mathrm{DC}}^{-1} \mathrm{C}_{\mathrm{AB}}^{-1}}{\left(\operatorname{det}\left(\mathrm{C}^{\mathrm{GAG}}\right)\right)^{2.25}}
$$

Combining like Terms

$$
=2.5 \frac{\alpha\left(\rho_{0}^{\mathrm{GAG}}\right)^{2.5} \mathrm{C}_{\mathrm{DC}}^{-1} \mathrm{C}_{\mathrm{AB}}^{-1}}{\left(\operatorname{det}\left(\mathrm{C}^{\mathrm{GAG}}\right)\right)^{1.25}}
$$

Therefore,

$$
\widetilde{\mathbb{C}}_{\mathrm{ABCD}}^{\mathrm{GAG}}=\left(\frac{\alpha\left(\rho_{0}^{\mathrm{GAG}}\right)^{2.5}}{\left(\operatorname{det}\left(\mathrm{C}^{\mathrm{GAG}}\right)\right)^{1.25}}\right)\left(C_{A C} C_{B D}+C_{A D} C_{B C}\right)+2.5 \frac{\alpha\left(\rho_{0}^{\mathrm{GAG}}\right)^{2.5} \mathrm{C}_{\mathrm{DC}}^{-1} \mathrm{C}_{\mathrm{AB}}^{-1}}{\left(\operatorname{det}\left(\mathrm{C}^{\mathrm{GAG}}\right)\right)^{1.25}}
$$

\section{A.4.3 PG Jacobian Stiffness Matrix}

To calculate the PG Jacobian stiffness matrix the result from section 3.4.1shown below is applied

$$
\widetilde{\mathbb{C}}_{\mathrm{ijkr}}^{\mathrm{ac}}=\frac{1}{\mathrm{~J}}\left[\left[\frac{\mathrm{J}}{2}\left(\delta_{\mathrm{ik}} \delta_{\mathrm{pr}}+\delta_{\mathrm{pk}} \delta_{\mathrm{ir}}\right) \mathrm{T}_{\mathrm{pj}}+\frac{\mathrm{J}}{2}\left(\delta_{\mathrm{jk}} \delta_{\mathrm{nr}}+\delta_{\mathrm{nk}} \delta_{\mathrm{jr}}\right) \mathrm{T}_{\mathrm{in}}\right]+\left[\widetilde{\mathbb{C}}_{\mathrm{ABCD}} \mathrm{F}_{\mathrm{iA}} \mathrm{F}_{\mathrm{jB}} \mathrm{F}_{\mathrm{kc}} \mathrm{F}_{\mathrm{rD}}\right] \mid\right.
$$

Applying the PG Cauchy stress equation derived in Appendix A.4.1 A.4.1 PG Cauchy Stress and the PG material elasticity tensor derived in Appendix A.4.2 the Abaqus FE implementable PG material Jacobian stiffness matrix is 


$$
\begin{aligned}
& \mathbb{C}_{\mathrm{ij} k \mathrm{kr}}^{\mathrm{GAG}}=\left[\frac{1}{2}\left(\delta_{\mathrm{ik}} \delta_{\mathrm{pr}}+\delta_{\mathrm{pk}} \delta_{\mathrm{ir}}\right) \mathbf{T}_{\mathrm{pj}}^{\mathrm{GAG}}+\frac{1}{2}\left(\delta_{\mathrm{jk}} \delta_{\mathrm{nr}}+\delta_{\mathrm{nk}} \delta_{\mathrm{jr}}\right) \mathbf{T}_{\mathrm{in}}^{\mathrm{GAG}}\right] \\
&+\frac{1}{\mathrm{~J}}\left[\left(\frac{\alpha\left(\rho_{0}^{\mathrm{GAG}}\right)^{2.5}}{\left(\operatorname{det}\left(\mathbf{c}^{\mathrm{GAG}}\right)\right)^{1.25}}\right)\left(C_{A C} C_{B D}+C_{A D} C_{B C}\right)\right. \\
&+\left.\left.2.5 \frac{\alpha\left(\rho_{0}^{\mathrm{GAG}}\right)^{2.5} \mathrm{C}_{\mathrm{DC}}^{-1} \mathrm{C}_{\mathrm{AB}}^{-1}}{\left(\operatorname{det}\left(\mathrm{c}^{\mathrm{GAG}}\right)\right)^{1.25}}\right] \mathrm{~F}_{\mathrm{iA}} \mathrm{F}_{\mathrm{jB}} \mathrm{F}_{\mathrm{kC}} \mathrm{F}_{\mathrm{rD}}\right]
\end{aligned}
$$

\section{A.4.4 Polyconvexity of PG Strain Energy Density Function}

Given:

$$
\begin{gathered}
W^{\mathrm{PG}}=\frac{2}{3} \alpha_{1} \frac{\left(\rho_{0}^{\mathrm{GAG}}\right)^{\alpha_{\mathrm{Z}}}}{J^{\left(\alpha_{2}-1\right)}} \\
\frac{\partial W^{P G}}{\partial J}=-\frac{2}{3} \alpha_{1}\left(\frac{\rho_{0}^{P G}}{J}\right)^{\alpha_{2}}
\end{gathered}
$$

Calculating the second derivative for,

$$
\frac{\partial^{2} W^{P G}}{\partial J^{2}}=\frac{2}{3\left(\alpha_{2}+1\right)} \alpha_{1}\left(\frac{\rho_{0}^{P G}}{J^{\left(\alpha_{2}+1\right)}}\right)
$$

Showing that if $\alpha_{1}>0, \alpha_{2}>0$ and $\alpha_{2} \neq 1$ then $\frac{\partial^{2} W^{2} G}{\partial J^{2}}>0$ and the strain energy function is polyconvex ${ }^{6}$.

\footnotetext{
${ }^{6}$ Note that the jacobian $\mathrm{J}$ is, by definition never $<0$, and for physically reasonable problems $p_{0}^{P G}>0$. These conditions are also necessary for polyconvexity of the $P G$ strain energy density function and are assumed during the derivation.
} 


\section{Appendix B: Data}

\section{B.5.1 Biochemical Data}

Table B.1. Experimentally measured biochemical parameters mean COL fiber volume fraction, $\phi_{\mathrm{f}}$ and mean Reference Configuration GAG density, $\rho_{0}^{\mathrm{PG}}$ for D0, D12: IGF-1, and D12: TGF- $\beta 1$ ( $S$ and $M$ Layers). COL volume fraction is calculated from (\%WWf) using the true density of $\mathrm{COL}$ as $1,436 \mathrm{~g} / \mathrm{cm}^{3}$ (Basser et al. 1998). These parameters were all group specific inputs for the FE model used to determine true COL fiber modulus and MAT shear modulus. Values from (Williams et al. 2010)

\begin{tabular}{|c|c|c|c|c|c|c|}
\hline Group & Layer & $\begin{array}{c}\text { Sample } \\
\text { size }(\mathrm{n})\end{array}$ & $\begin{array}{c}\Phi_{\mathrm{f}} \\
\% \text { of total tissue } \\
\text { volume })\end{array}$ & $\begin{array}{c}\phi_{\mathrm{f}} \\
\text { Standard } \\
\text { Deviation }\end{array}$ & $\begin{array}{c}\rho_{\mathrm{o}}^{P G} \\
(\mathrm{mg} / \mathrm{ml})\end{array}$ & $\begin{array}{c}\rho_{0}^{P G} \\
\text { Standard } \\
\text { Deviation }\end{array}$ \\
\hline D0 & Superficial & 16 & 3.85 & 0.968 & 32.8 & 7.50 \\
\hline D0 & Middle & 18 & 4.98 & 1.44 & 47.6 & 12.1 \\
\hline D12: IGF-1 & Superficial & 15 & 3.32 & 1.00 & 30.5 & 4.30 \\
\hline D12: IGF-1 & Middle & 11 & 4.01 & 2.09 & 33.0 & 9.20 \\
\hline D12: TGF- $\beta 1$ & Superficial & 12 & 5.39 & 1.08 & 41.8 & 6.90 \\
\hline D12: TGF- $\beta 1$ & Middle & 16 & 5.83 & 1.59 & 51.3 & 12.8 \\
\hline
\end{tabular}




\section{B.5.2 Experimental Tensile Data}

Table B.2. Experimentally measured mean Tensile modulus, $\mathrm{E}^{+}$for D0, D12: IGF-1, and D12: TGF- $\beta 1$ ( $S$ and M Layers). Ridge Tensile modulus was used to validate/fit the FE model to experimental data for each group. Note that values represent the averaged strain $(7.5 \%)$ of both $5 \%$ and $10 \%$ experimental strain levels. (Stender et al. 2011).

\begin{tabular}{|c|c|c|c|c|c|c|c|c|}
\hline Group & Layer & $\begin{array}{c}\text { Sample } \\
\text { size (n) }\end{array}$ & $\begin{array}{c}\mathrm{E}^{+} \\
7.5 \% \\
\text { strain } \\
\text { Groove } \\
(\mathrm{MPa})\end{array}$ & $\begin{array}{c}\mathrm{E}^{+} \text {Groove } \\
\text { Standard } \\
\text { Deviation }\end{array}$ & $\begin{array}{c}\text { Groove to } \\
\text { Ridge } \\
\text { Conversion } \\
\text { factor }\end{array}$ & $\begin{array}{c}\mathrm{E}^{+} \\
\begin{array}{c}7.5 \% \\
\text { strain } \\
\text { Ridge } \\
\text { (MPa) }\end{array}\end{array}$ & $\begin{array}{c}\mathrm{E}^{+} \\
\text {Ridge } \\
\text { Standard } \\
\text { Deviation }\end{array}$ & $\begin{array}{c}\text { Mean tare } \\
\text { strain } \\
(\mathrm{mm} / \mathrm{mm})\end{array}$ \\
\hline D0 & Superficial & 10 & 3.87 & 1.29 & $\mathrm{~N} / \mathrm{A}$ & $1.81^{*}$ & 0.687 & 0.028 \\
\hline D0 & Middle & 14 & 14.2 & 9.50 & $\mathrm{~N} / \mathrm{A}$ & $5.67^{*}$ & 1.78 & 0.026 \\
\hline D12: IGF-1 & Superficial & 12 & 0.426 & 0.253 & 0.470 & 0.200 & 0.119 & 0.154 \\
\hline D12: IGF-1 & Middle & 12 & 0.714 & 0.528 & 0.410 & 0.293 & 0.217 & 0.373 \\
\hline D12: TGF- $\beta 1$ & Superficial & 12 & 8.69 & 1.63 & 0.470 & 4.08 & 0.768 & 0.021 \\
\hline D12: TGF- $\beta 1$ & Middle & 14 & 12.0 & 6.84 & 0.410 & 4.908 & 2.80 & 0.04 \\
\hline
\end{tabular}

\section{B.5.3 Experimental Confined Compression}

\section{Data}

Table B.3. Experimentally measured mean CC modulus, $\mathbf{H}_{A}$ for D0, D12: IGF-1, and D12: TGF- $\beta 1$ ( $S$ and M Layers). The CC modulus was used to validate the FE model to experimental data for each group. Note that values represent the average strain (22.5\%) of both $15 \%$ and $30 \%$ experimental strain levels. Data from (Williams et al. 2010).

\begin{tabular}{|c|c|c|c|c|}
\hline \multirow{2}{*}{ Group } & Layer & Sample size $(\mathrm{n})$ & $\begin{array}{c}\mathrm{H}_{\mathrm{A}} 22.5 \% \text { CC strain } \\
(\mathrm{MPa})\end{array}$ & $\begin{array}{c}\mathrm{H}_{\mathrm{A}} \\
\text { Standard Deviation }\end{array}$ \\
\hline D0 & Superficial & 20 & 0.115 & 0.068 \\
\hline D0 & Middle & 30 & 0.300 & 0.150 \\
\hline D12: IGF-1 & Superficial & 26 & 0.04 & 0.037 \\
\hline D12: IGF-1 & Middle & 20 & 0.08 & 0.07 \\
\hline D12: TGF- $\beta 1$ & Superficial & 24 & 0.219 & 0.116 \\
\hline D12: TGF- $\beta 1$ & Middle & 18 & 0.32 & 0.13 \\
\hline
\end{tabular}




\section{B.5.4 Experimental Unconfined Compression}

\section{Data}

Table B.4. Experimentally measured mean UCC modulus, $\mathrm{E}^{-}$and Poisson's Ratio, $v$ for D0, D12: IGF-1, and D12: TGF- $\beta 1$ (S and M Layers). $\mathbf{E}^{-}$and $v$ are used to validate/fit the FE model to experimental data for each group. Note that values represent the average strain $(22.5 \%)$ of both $15 \%$ and $30 \%$ experimental strain levels. Data from (Williams et al. 2010).

\begin{tabular}{|c|c|c|c|c|c|c|c|}
\hline Group & Layer & $\begin{array}{c}\mathbf{E}^{-} \\
\text {Sample } \\
\text { size (n) }\end{array}$ & $\begin{array}{c}\mathbf{E}^{-} \text {22.5\% } \\
\text { UCC strain } \\
(\mathrm{MPa})\end{array}$ & $\begin{array}{c}\mathbf{E}^{-} \\
\text {Standard } \\
\text { Deviation }\end{array}$ & $\begin{array}{c}\boldsymbol{v} \\
\text { Sample } \\
\text { size (n) }\end{array}$ & $\begin{array}{c}\boldsymbol{v} 22.5 \% \\
\text { UCC strain } \\
(\mathrm{MPa})\end{array}$ & $\begin{array}{c}\boldsymbol{v} \\
\text { Standard } \\
\text { Deviation }\end{array}$ \\
\hline D0 & Superficial & 22 & 0.131 & 0.070 & 44 & 0.077 & 0.046 \\
\hline D0 & Middle & 14 & 0.228 & 0.108 & 28 & 0.123 & 0.122 \\
\hline D12: IGF-1 & Superficial & 20 & 0.022 & 0.022 & 28 & 0.191 & 0.042 \\
\hline D12: IGF-1 & Middle & 22 & 0.031 & 0.046 & 40 & 0.0209 & 0.171 \\
\hline D12: TGF- $\beta 1$ & Superficial & 18 & 0.223 & 0.110 & 34 & 0.066 & 0.064 \\
\hline D12: TGF- $\beta 1$ & Middle & 18 & 0.363 & 0.068 & 36 & 0.058 & 0.048 \\
\hline
\end{tabular}




\section{B.5.5 Mechanical Constants}

Table B.5. Numerical values for mechanical constants true COL fiber modulus $E_{f}(M P a)$ and MAT shear modulus, $\mu$ (MPa) as determined by three fitting methods to experimental data.

\begin{tabular}{|c|c|c|c|c|c|c|c|}
\cline { 3 - 8 } \multicolumn{2}{c|}{} & \multicolumn{2}{c|}{ Fit to UCC } & \multicolumn{2}{c|}{ Fit to Tensile } & \multicolumn{2}{c|}{ Simultaneous fit } \\
\hline Group & Layer & $\begin{array}{c}\text { True COL } \\
\text { fiber } \\
\text { modulus, } \\
\mathrm{E}_{\mathrm{f}}(\mathrm{MPa})\end{array}$ & $\begin{array}{c}\text { MAT } \\
\text { shear } \\
\text { modulus, } \\
\mu(\mathrm{MPa})\end{array}$ & $\begin{array}{c}\text { True COL } \\
\text { fiber } \\
\text { modulus, } \\
\mathrm{E}_{\mathrm{f}}(\mathrm{MPa})\end{array}$ & $\begin{array}{c}\text { MAT } \\
\text { shear } \\
\text { modulus, } \\
\mu(\mathrm{MPa})\end{array}$ & $\begin{array}{c}\text { True COL } \\
\text { fiber } \\
\text { modulus, } \\
\mathrm{E}_{\mathrm{f}}(\mathrm{MPa})\end{array}$ & $\begin{array}{c}\text { MAT } \\
\text { shear } \\
\text { modulus, } \\
\mu(\mathrm{MPa})\end{array}$ \\
\hline D0 & Superficial & 370 & 0.001 & 290 & 0.001 & 320 & 0.001 \\
\hline D0 & Middle & 320 & 0.001 & 710 & 0.001 & 550 & 0.001 \\
\hline D12: IGF-1 & Superficial & 90 & 0.001 & 26 & 0.001 & 40 & 0.001 \\
\hline D12: IGF-1 & Middle & 80 & 0.001 & 34 & 0.001 & 46 & 0.001 \\
\hline D12: TGF- $\beta 1$ & Superficial & 530 & 0.001 & 480 & 0.001 & 540 & 0.001 \\
\hline D12: TGF- $\beta 1$ & Middle & 950 & 0.001 & 490 & 0.001 & 690 & 0.001 \\
\hline
\end{tabular}

\section{B.5.6 Mesh Convergence Study}

In order to validate the single element models developed for this study, a mesh convergence study was performed for a DO S layer specimen for CC and Tension models. Stretch following the equilibrium step, as well as the equilibrium Cauchy was recorded for $7.5 \%$ tensile strain and $22.5 \%$ UCC strain. Element size was varied to create models with 1, 8, 64, and 512 elements. As expected, mesh size had no effects on the FEA results. The results are shown in figures B1 and B2. Note that these values may not correspond to validation results due to the exclusion of tare strain and/or variation in material parameters. 




Figure B.1. Mesh Convergence study for the D0 S layer group in tension (7.5\% strain) and UCC (22.5\% strain)

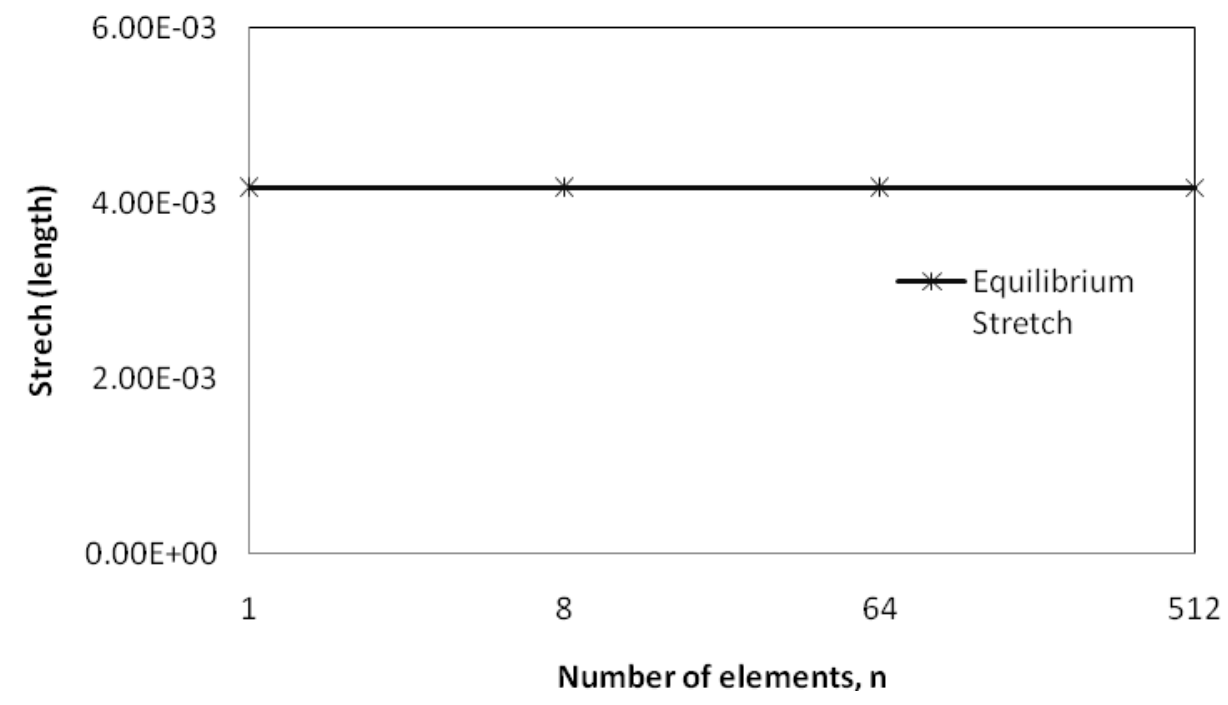

Figure B.2. Mesh convergence study results for theD0 S Layer group for initial stretch following the SM equilibrium step. 